\title{
Calculated Neutron KERMA Factors Based on the LLNL ENDL Data File
}

\author{
R. J. Howerton \\ Manuscript date: January 1986
}

\author{
DISCLAIMER
}

\begin{abstract}
This report was prepared as an account of work sponsored by an agency of the United States Governmemt. Neither the United States Government nor any agency thereor, nor any of their employees, makes any warranty, express or implied, or assumes any legal liability or responsibility for the accuracy, completeness, or usefulness of any information, apparatus, product, or process disclowed, or sepresents that its use would not infringe privately owned rights. Reference herein to any specific commercial produch, process, or service by trade name, trademark. manufacturer, or otherwise does not necessarily constitute or imply its endorsement, recommendation, or favoring by the Uniled States Government or any agency thereof. The views and opinions of authors expressed herein do not necessarily state or reflect those of the United Stutes Government or any agency thereof.
\end{abstract}

\section{LAWRENCE LIVERMORE NATIONAL LABORATORY University of California - Livermore, California - 94550}




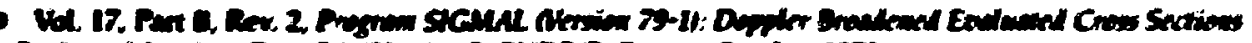

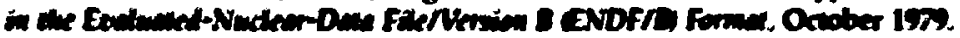

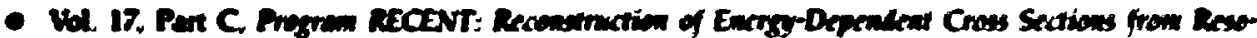

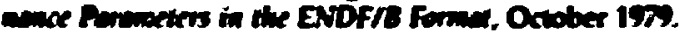

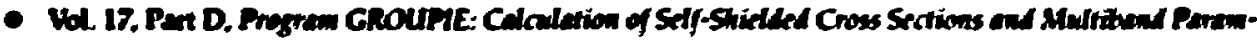

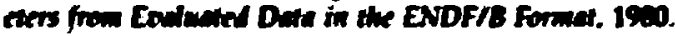

- Vol 17. Pat E, Program EVALPLT: Plot Data in the Eoduaded-Nuctear-Deta File/Version a ENDFI Do Fonnes. February 1979. 1979.

- Vol. 17. Pant F, DOWNER Nersion 79-1): Growp Collepse Cross Section end Transfer Marrices, lanuary

- Vol. 18, ACTL. Emaluated Neutron Actioation Cross-Section Librery October 1978.

- Vo1. 19, Newtron-Induced Angular and Emergy Distributions: Graphical Experimental Data, April 1977.

- Vol. 20, Bonderenko Self-Shielded Cross Sections and Multiband Parameters Derized from the UL Exaluated-Nuclear-Date Library (ENDU. July 1978.

- Vol. 21. Part A, Maxiell-Averaged Reactions Rates (ow) for Selected Reactions beticcen lous arith Atomic Mass $\leq 11$. February 1979.

- Vol. 21. Part C, Program SIGMAL Nersion 79-1): Doppler-Broadened Enaluated Cross Sections in the Liocrmore-Evaluated Nuclear Dafa Library (ENDL) Format, March 1979.

- Vol. 22. Rev. 1, GAMIDEN: A Program to Aid in the Identification of Unkuniwn Materials by GamniaRay Spectroscopy. June 1982.

- Vol. 23, ENSL and CDRL: Evaluated Nuclear Structure Librarics. February 1981.

- Vol. 23, Addendum, ENSL82 and CDRL82: The 1982 Version of Exaluated Nuclear Structure Libraries. January 1983.

- Vol. 24, Thresholds and Q Valucs of Nuclear Reactions Induced by Neutrons. Protons, Deuterons. Tritons, ${ }^{3} \mathrm{He}$ lons, Alpha Particles, and Photons, March 1981.

- Vol. 25, OMEGA: A CRAY 1 Executide Code for LLNL Nuclear Data Libraries, August 1983.

- Vol. 26, A Bibliography and Index for Nuclear Reactions Among Light Charged Particles, September 1984.

- Vol. 27, Calculated Neutron KERMA Factors Based on the LLNL ENDL Data File, Jamuary 1986. 


\section{Condeats}

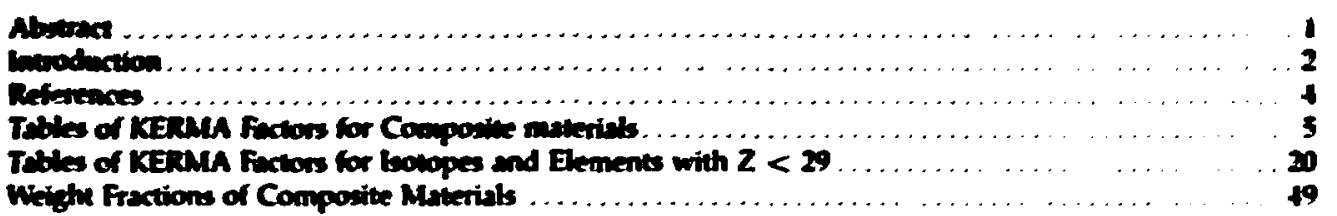




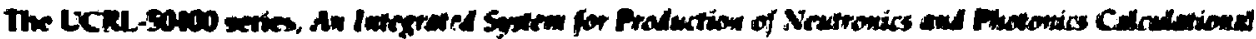

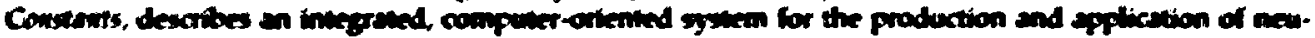

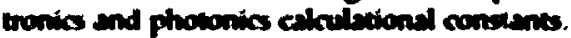

The svsem supplies reliatle, up-wo-dse data, selects specific types of dara on requex, provides

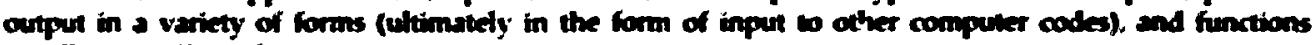
rapidy and efficiently.

The URRL-50500 serin s comprises the following volumes:

- Vol. I. Pan A, Rev. 3, ECSIL. A System for Storege. Retriesal. and Display of Experimental Nerutrom Data. September 1976.

- Vol. I. Part B. Program ECSX\& Nersion 78-11: Contersion of Expermentally Measured Cross-Srction Dasa frem the four-Center-Exchange $\alpha-4$ Format to the Litermorc ECSIL formet. December 1978.

- Vol. 2, Rev. 2, A Bibliography of the Expcrimentel Data of Neutron-Induced interactions. July 1976.

- Vol. 3, Rev. 2, An Index of the Experimental Date of Neutran-Induced Interactians. July 1976.

- Vol. 4. Rev. 1, Evaluated Nuclear Data Library, September 1981.

- Vol. 4, Rev. 1. Appendix C. The Neutron Library (ENDL82) in the Transmittal Format. June 1982.

- Vol. 5. Part A. Rev. 1. CLYDE: A Code for the Production of Calculational Constants from Nucleas Data. September 1975.

- Vol 5, Part B, Rev. 1. Relativistic Transformations heticeen Center-of-Mass and Laboratory Sỵstems for Two-Body Nuclear Reactions. April 1978.

- Vol. 6. Rev. 2. Tahles and Graphs of Photon-Interaction Cross Sections from $1 \mathrm{kcV}$ to $100 \mathrm{McV}$. December 1978.

- Vol. 7. Part A, Rev. 1, Major Neutron-Induced Interactions $Z \leq$ 55): Graphical, Experimental Data, July 1976.

- Vol. 7, Part B, Rev. 1, Major Neutron-Induced Interactions (Z > 55): Graphical, Experimental Data. July 1976.

- Vol. 8, Part A, Rev. 1, Supplemental Ncutron-Induced Interactions $(Z \leq 35)$ : Graphical, Experimental Data, July 1976

- Vol. 8, Past B, Rev. 1, Supplemental Neutron-Induced Interactions $(Z>35)$ : Graphical, Experimental Data, July 1976.

- Vol. 9, Thresitolds of Nuclear Reactions Induced by Neuhons, Photons, Detuterons. Tritons, and Alpha Particles, September 1970.

- Vol. 10, Rev. 1, Tabulated Experimental Data for Neutron-Induced Interactions, July 1976.

- Vol. 11, Experimental Data, Indexes, and Techmiques of Obtaining a Selected Set of Neutron Resonance Parameters, May 1972.

- Vol. 12, An Atlas of Resolved Neutron Resonance Parameters, July 1972.

- Vol. 13, An Atlas of Unresolved Neutron Resonance Parameters, September 1972.

- Vol. 14, TARTNP: A Conpled Neutroit-Phofon Montc Carlo Transport Code, February 1976.

- Vol. 15. Part A. The LLL Evaluated-Nuclear-Dafa Library (ENDL): Evaluation Techniques, Reaction Index, and Descriptions of Individual Evaluations, September 1975.

- Vol. 15, Part B, Rev. 1. The LLL Evaluated-Nuclear-Data Library (ENDL): Graphs of Cross Sections from the Library, October 1978.

- Vol. 15, Part C, The LLL Evaluated-Nuclear-Data Library (ENDL): Translation of ENDL NeutronInduced Interaction Data into the ENDF/B Format, April 1976.

- Vol. 15, Part D, Rev. 1, The LLL Evaluated-Nuclear-Data Library (ENDL): Descriptions of Individual Evaluations for $Z=0-98$. May 1978.

- Vol. 15, Part E, Data Testing Results for the LLL Nuclear Data Library (ENDL-78), August 1979.

- Vol. 15, Part F, Experimental and Evaluated Elastic Nuclear Plus Interference Cross Sections for Light Charged Particles, July 1980.

- Vol. 16, Rev. 2, Tabular and Graphical Presentation of 175 Neutron-Group Constants Derived from the LLL Evaluated-Nuclear-Data Library (ENDL, October 1978.

- Vol. 17, Part A, Rev. 2, Programi LINEAR (Version 79-1): Linearize Data in the Evaluated-NuclearData File/Version B (ENDF/B) Format, October 1979. 


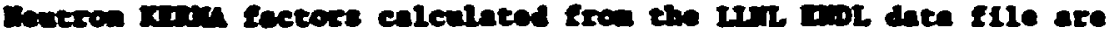
tabulated for 15 compolte meerlals and for che lsotopas or alements in

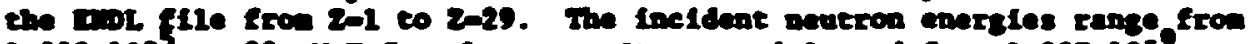
$1.002 \times 10^{-5}$ to 20 . Mot for the composite enterials and from $1.307 \times 10^{-9}$ to 20. Ev for the Isotopes and elements. 


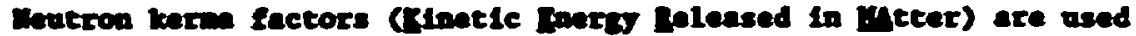
extensively in blonodical applications and for deternining beating in naterinls of interest fron beutron-Induced reactions in fission or fusion power applications. The enery roleased is obtained by forming the product of the enerey-dependent neutron fluance and the fluence-areraged keran factors for the meterial under study. Generally, the kerna for a neutron-Induced reaction is defined to be the energy avallable $\left(B_{p}+Q\right)$ less the energy carried oif by secondary neutrons and photons. The kerme for a aterial is then obtelned by swaing the kerans of the indiridual reactions. properis velghted by Isotopic or elemental abundance in the case of couposite anterials.
\end{abstract}

A considerable body of Ifterature dealing with both calculated and experimental determinations of kerma factors exists. In 1980, Caswell, Coyne and Randolph ${ }^{1}$ discussed the methods used to calculate kerma factors from files of evaluated neutron-Induced reaction data. They made the point that "The chlef problem in carrying out this kind of calculation is supplying needed nuclear data which are not in the file." In particular, the main problem to which they alluded was the lack of explicit energy distributions for secondary charged particles from neutron-Induced reactions. In 1975, Abdou and Maynard ${ }^{2}$ had called attention to "... Inconsistency in preserving the energy between the basic neutron interaction data and the gamma-ray production data."

In 2981, explictt energy distributions for all secondary particles from all neutron-induced reactions were either entered into the LLNL ENDL $^{3}$ data files or were derivable by simple kinematics equations from angular distributions in the cases of two-body reactions with well-defined Q-Values and final-state definitions. With these additions to the data files, it was possible to Insure energy conservation between neutron interaction and neutron-Induced gamma-ray production data. The current tolerance for energy conservation in these files is 58 or $100 \mathrm{keV}$, whichever is the lesser.

The tables of kerma factors presented here were calculated from the current version of the ENDL data file (dated December 27, 1985). The results are presented for the 175 neutron energy groups used by the TART ${ }^{4}$ Monte Carlo neutron transport code for the isotopes and elemental mixtures. For the composite materlals, a subset of the TART groups that eliminated neutron energies below the molecular binding energies where diffurent reaction kinematics are required than those of isolated nuclei. There are two reasons for using the TART groups. One is that LLNL users of the kerma factors will find this convenient and the other is that it is 


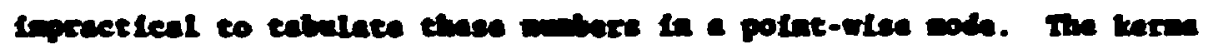

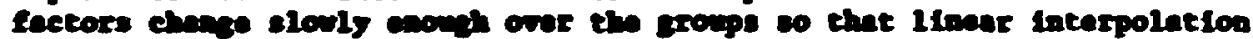

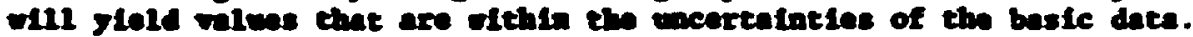
since the calculated kare tectors ware derived eatiraly fron the

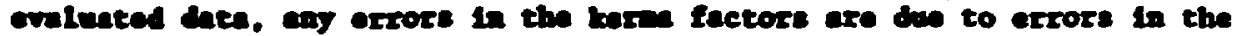
ovelweted date. Inws, the calculated velues an be conpared wth experimentel kerm tactors and sorve to test the validity of the EDL date Elies. 


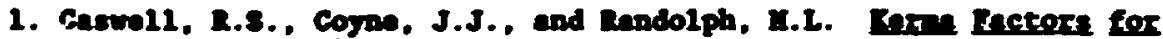

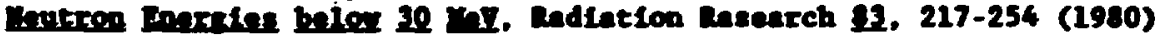

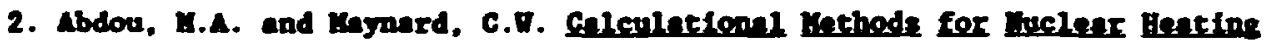
Rert I: Theoreticel and comentionel Aleortthen, Huclear science and Engineering 56. 360-380 (1975)

3. Howerton, R.J., Dye, R.E., and Perkins, S.T. Bvaluated puclenr Dete Librery. UCRL 50400 Vol. 4 Revision 1 (1981). See also Volunes 15 Parts A through $E$ and Volume 25 of this Series.

4. Plechaty, E.F. . and Kimlinger, J.R. TARTHP: A coupled neutron-photon Yonte Garlo transport code, DCRL 50400 Vol. 14 (1976) Note: The name of this code was subsequently changed to TART.

* Work performed under the auspices of the U.S. Department of Energy by the Lawrence Livermore National Laboratory under contract number W-7405-ENG-48. 


\section{$\tan ^{2}$}

\section{Daers \\ (12v) \\ TIeses \\ (Approx.)}

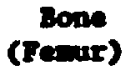

Hasele

(ICRD)
Standard Inn

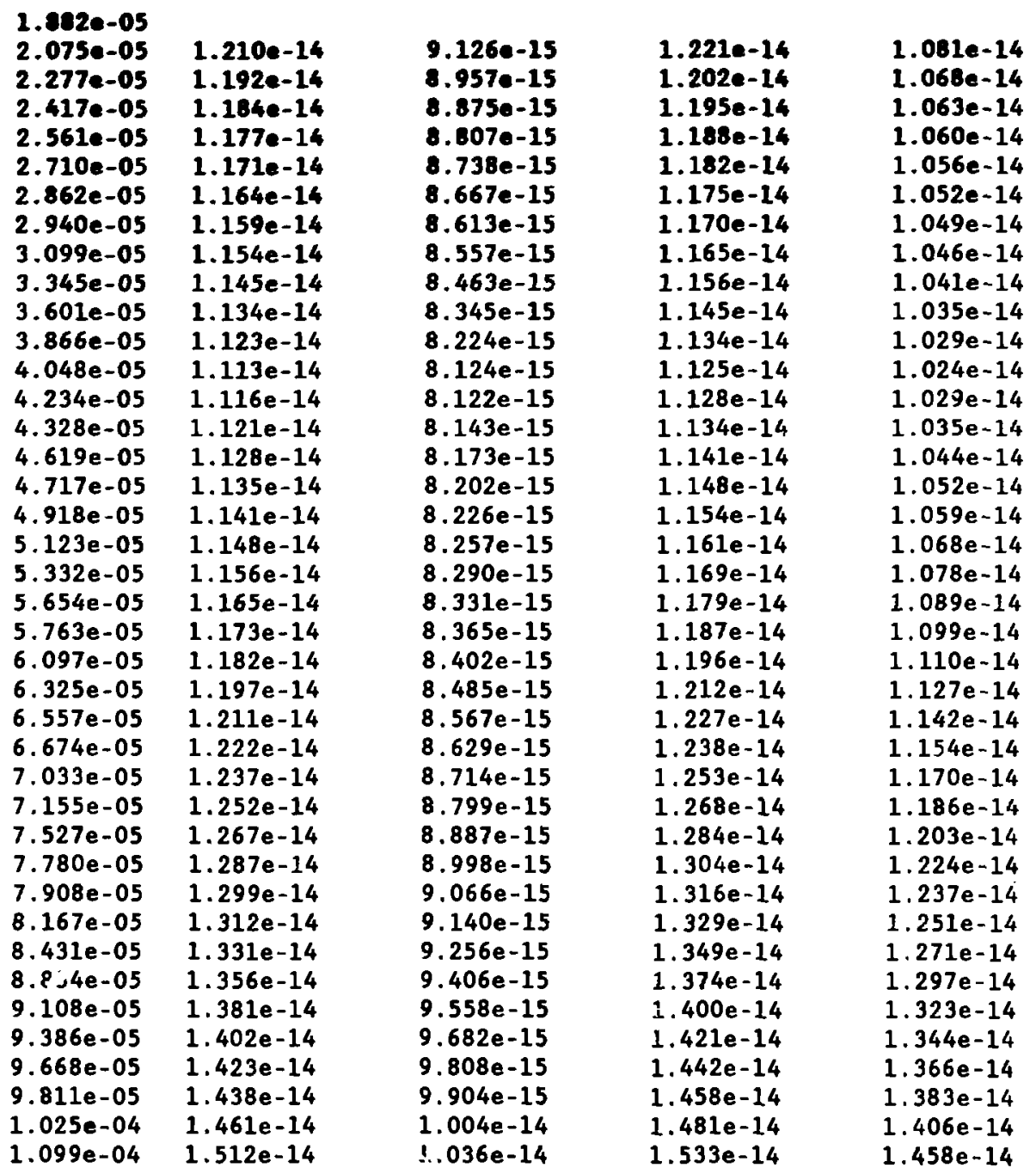




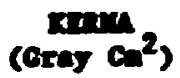

Inerty
(itev)

$\begin{array}{ll}1.176 e-04 & 1.578 e-14 \\ 1.256 e-04 & 1.647 e-14 \\ 1.338 e-04 & 1.718 e-14 \\ 1.406 e-04 & 1.783 e-14 \\ 1.511 e-04 & 1.859 e-14 \\ 1.601 e-04 & 1.944 e-14 \\ 1.694 e-04 & 2.024 e-14 \\ 1.789 e-04 & 2.105 e-14 \\ 1.887 e-04 & 2.190 e-14 \\ 1.988 e-04 & 2.276 e-14 \\ 2.091 e-04 & 2.368 e-14 \\ 2.741 e-04 & 2.726 e-14 \\ 3.267 e-04 & 3.285 e-14 \\ 3.811 e-04 & 3.793 e-14 \\ 4.704 e-04 & 4.480 e-14 \\ 4.991 e-04 & 5.053 e-14 \\ 5.658 e-04 & 5.518 e-14 \\ 6.043 e-04 & 6.029 e-14 \\ 6.367 e-04 & 6.375 e-14 \\ 7.156 e-04 & 6.920 e-14 \\ 8.322 e-04 & 7.876 e-14 \\ 9.177 e-04 & 8.865 e-14 \\ 1.058 e-03 & 9.972 e-14 \\ 1.307 e-03 & 1.188 e-13 \\ 1.581 e-03 & 1.444 e-13 \\ 1.882 e-03 & 1.727 e-13 \\ 2.208 e-03 & 2.034 e-13 \\ 2.561 e-03 & 2.368 e-13 \\ 2.940 e-03 & 2.726 e-13 \\ 3.345 e-03 & 3.109 e-13 \\ 3.777 e-03 & 3.517 e-13 \\ 4.234 e-03 & 3.949 e-13 \\ 5.763 e-03 & 4.905 e-13 \\ 7.527 e-03 & 6.448 e-13 \\ 1.025 e-02 & 8.513 e-13 \\ 1.511 e-02 & 1.188 e-12 \\ 2.091 e-02 & 1.634 e-12 \\ 3.646 e-02 & 2.087 e-12 \\ 3.953 e-02 & 2.987 e-12 \\ 4.704 e-02 & 3.464 e-12\end{array}$

\author{
Done \\ (Pewar)
}

$1.078=-14$

1. $121 \mathrm{e}-14$

1. $166 e-14$

1.207 e-14

$1.255 e-14$

1. 308e-14

$1.358 e-14$

1. $410 e-14$

$1.463 e-14$

$1.517 d-14$

$1.576 \mathrm{e}-14$

$1.806 \mathrm{e}-14$

$2.165 e-14$

$2.491 \mathrm{e}-14$

$2.933 e-14$

3. $303 e-14$

$3.603 \mathrm{e}-14$

$3.934 \mathrm{e}-14$

4. $157 e-14$

4. $510 \mathrm{e}-14$

$5.128 e-14$

$5.768 e-14$

$6.485 e-14$

7. 718 e- 14

$9.381 e-14$

1. 121e-13

1. $320 \mathrm{e}-13$

$1.537 e-13$

$1.769 e-13$

2. $017 \mathrm{e}-13$

$2.282 e-13$

2. 561e-13

$3.182 \mathrm{e}-13$

4. $184 e-13$

$5.527 e-13$

$7.711 \mathrm{e}-13$

1. $061 \mathrm{e}-12$

1. $356 e-12$

$1.645 e-12$

$1.943 e-12$

2. $253 e-12$ unsele (ICID)

\section{601e-14}

$1.671 \mathrm{e}-14$

$1.743 e-14$

$1.810 \mathrm{e}-14$

$1.887 \mathrm{e}-14$

$1.973 e-14$

$2.054 e-14$

$2.138 e-14$

$2.223 e-14$

2.311e-14

$2.405 \mathrm{e}-14$

$2.769 e-14$

$3.338 \mathrm{e}-14$

3.854e-14

$4.553 e-14$

5.135e-14

$5.608 e-14$

$6.128 \mathrm{e}-14$

$6.479 \mathrm{e}-14$

$7.033 \mathrm{e}-14$

8. $005 e-14$

$9.011 \mathrm{e}-14$

$1.014 \mathrm{e}-13$

1. $207 e-13$

1. $458 \mathrm{e}-13$

$1.755 e-13$

$2.068 \mathrm{e}-13$

$2.407 e-13$

$2.773 e-13$

$3.261 e-13$

$3.575 e-13$

4. $014 e-13$

$4.987 e-13$

6. $554 \mathrm{e}-13$

8. $652 \mathrm{e}-13$

$1.207 \mathrm{e}-12$

1. 661 e -12

2. $121 \mathrm{e}-12$

2. $570 \mathrm{e}-12$

$3.035 e-12$

3. $520 \mathrm{e}-12$ senndard inn

$1.526 e-14$

$1.596 e-14$

$1.668 \mathrm{e}-14$

1.735 e- 14

1.811e-14

$1.898 \mathrm{e}-14$

$1.979 e-14$

$2.063 e-14$

2. $148 \mathrm{e}-14$

2. $236 \mathrm{e}-14$

2. $329 \mathrm{e}-14$

2. $688 \mathrm{e}-14$

3. 250e-14

$3.760 \mathrm{e}-14$

4. $449 \mathrm{e}-14$

$5.023 e-14$

$5.488 e-14$

$6.000 \mathrm{e}-14$

6. $346 \mathrm{e}-14$

$6.890 \mathrm{e}-14$

$7.846 e-14$

$8.834 e-14$

$9.940 e-14$

1. $184 \mathrm{e}-13$

1. $440 \mathrm{e}-13$

1. $722 \mathrm{e}-13$

$2.030 \mathrm{e}-13$

2. $363 \mathrm{e}-13$

2. $724 \mathrm{e}-13$

3. $104 \mathrm{e}-13$

3. $510 \mathrm{e}-13$

3. $940 \mathrm{e}-13$

$4.896 \mathrm{e}-13$

$6.435 e-13$

$8.496 \mathrm{e}-13$

$1.185 e-12$

1. $631 e-12$

$2.083 \mathrm{e}-12$

2. $525 e-12$

2. $981 \mathrm{e}-12$

$3.457 e-12$ 


\section{$\operatorname{ton}_{\left(\operatorname{cras} \theta^{2}\right)}$}

\section{Enery (2iv) \\ TLexe \\ (Approx.)}

4.021e-12

4.672e-12

5. 337e-12

6. 020e-12

7.016e-12

8. 506e-12

9. 69 je-12

$1.052 \mathrm{e}-11$

1. $134 \mathrm{e}-11$

$1.198 e-11$

1. $270 \mathrm{e}-11$

1. $370 \mathrm{e}-11$

1. $504 \mathrm{e}-11$

1. $626 \mathrm{e}-11$

$1.679 e-11$

$1.853 e-11$

2. 019e-11

2. $315 \mathrm{e}-11$

2. $424 e-11$

2. $549 e-11$

2. $618 \mathrm{e}-11$

2. $770 \mathrm{e}-11$

2. $904 \mathrm{e}-11$

3. 047e-11

3. $114 e-11$

3. $190 \mathrm{e}-11$

3. $383 e-11$

3. $558 \mathrm{e}-11$

3. $785 e-11$

$4.107 e-11$

$4.183 e-11$

$4.190 e-11$

4. $352 \mathrm{e}-11$

4. 351e-11

$4.376 e-11$

$4.524 \mathrm{e}-11$

$4.476 \mathrm{e}-11$

4. 601e-11

4.631e-11

$4.752 \mathrm{e}-11$

$4.789 e-11$

\author{
Done \\ (Fenur)
}

2. 618e-12

3. 044e-12

3. $480 \mathrm{e}-12$

3. 3 j1e-12

4. 584e-12

5. 573e-12

6. $346 e-12$

6. $904 e-12$

$7.463 e-12$

7. $863 e-12$

8. $339 e-12$

8. $996 \mathrm{e}-12$

9.829e-12

$1.065 e-11$

1. $111 e-11$

$1.227 \mathrm{e}-11$

$1.336 \mathrm{e}-.11$

1. $520 \mathrm{e}-11$

$1.596 \mathrm{e}-11$

$1.683 \mathrm{e}-11$

1. $737 \mathrm{e}-11$

1. $836 \mathrm{e}-11$

$1.928 \mathrm{e}-11$

$2.033 e-11$

$2.074 e-11$

$2.135 e-11$

2. $275 \mathrm{e}-11$

$2.439 e-11$

$2.562 e-11$

$2.828 e-11$

$2.894 e-11$

$2.881 e-11$

$2.993 e-11$

2. $978 e-11$

$2.981 \mathrm{e}-11$

$3.073 e-11$

3. 061e-11

3. $125 \mathrm{e}-11$

3. $188 \mathrm{e}-11$

3. 211e -11

$3.230 \mathrm{e}-11$
Huscle
(ICxO)

4.086e-12

4.747e-12

$5.421 e-12$

6.115e-12

$7.127 e-12$

8.638e-12

$9.841 \mathrm{e}-12$

$1.068 \mathrm{e}-11$

1.15ie-11

1. 216e-11

1. $289 \mathrm{e}-11$

1. $390 e-11$

1. $527 \mathrm{e}-11$

$1.650 \mathrm{e}-11$

$1.703 e-11$

$1.880 e-11$

$2.048 \mathrm{e}-11$

2. $349 e-11$

$2.459 e-11$

$2.586 e-11$

$2.656 e-11$

2. 810e-11

$2.946 e-11$

3. $089 e-11$

3. $159 e-11$

3. $236 \mathrm{e}-11$

$3.430 e-11$

$3.600 e-11$

$3.836 \mathrm{e}-11$

4.154e-11

4. $231 \mathrm{e}-11$

$4.243 e-11$

$4.406 e-11$

$4.40 \mathrm{Be}-11$

$4.437 e-11$

$4.589 \mathrm{e}-11$

$4.539 e-11$

$4.669 e-11$

4.695e-11

$4.826 \mathrm{e}-11$

$4.866 \mathrm{e}-11$
Stendard

inn

4. $013 e-12$

4.663e-12

5.326e-12

$6.008 e-12$

$7.002 e-12$

$8.488 \mathrm{e}-12$

$9.669 e-12$

$1.049 \mathrm{e}-11$

1. 131e-11

1. $194 \mathrm{e}-11$

1. 266e-11

$1.363 e-11$

$1.493 e-11$

$1.614 \mathrm{e}-11$

$1.676 e-11$

1. $849 e-11$.

2. $013 e-11$

2. $296 \mathrm{e}-11$

$2.408 \mathrm{e}-11$

$2.536 e-11$

2. $611 \mathrm{e}-11$

2.762e-11

2. $896 \mathrm{e}-11$

3. $040 \mathrm{e}-11$

3. 111e-11

$3,193 t-11$

3. $382 e-11$

$3.563 e-11$

$3,775 e-11$

$4.092 e-11$

$4.172 e-11$

$4.180 e-11$

4. $337 e-11$

4. $340 e-11$

4. $366 e-11$

4. $507 \mathrm{e}-11$

4. $472 e-11$

$4.588 \mathrm{e}-11$

4. $629 \mathrm{e}-11$

4. $732 e-11$

$4.769 e-11$ 


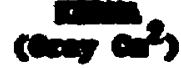

\begin{tabular}{|c|c|c|c|c|}
\hline 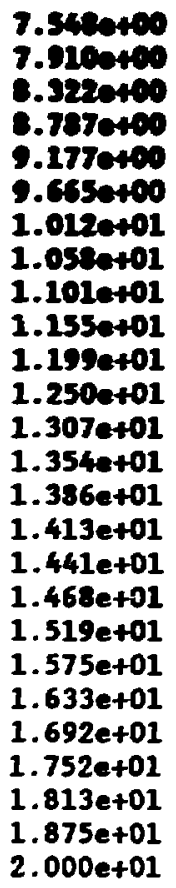 & $\begin{array}{l}5.025 e-11 \\
5.025 e-11 \\
5.009 e-11 \\
5.041 e-11 \\
5.215 e-11 \\
5.104 e-11 \\
5.200 e-11 \\
5.34 e-11 \\
5.442 e-11 \\
5.626 e-11 \\
5.767 e-11 \\
5.317 e-11 \\
5.923 e-11 \\
6.006 e-11 \\
6.071 e-11 \\
6.109 e-11 \\
6.151 e-11 \\
6.196 e-11 \\
6.267 e-11 \\
6.331 e-11 \\
6.401 e-11 \\
6.445 e-11 \\
6.463 e-11 \\
6450 e-11 \\
6.486 e-11 \\
6.582 e-11\end{array}$ & $\begin{array}{l}3.457 e-11 \\
3.541 e-11 \\
3.538 e-11 \\
3.496 e-11 \\
3.595 e-11 \\
3.643 e-11 \\
3.709 e-11 \\
3.761 e-11 \\
3.84 e-11 \\
4.015 e-11 \\
4.123 e-11 \\
4.194 e-11 \\
4.281 e-11 \\
4.357 e-11 \\
4.414 e-11 \\
4.451 e-11 \\
4.487 e-11 \\
4.526 e-11 \\
4.595 e-11 \\
4.663 e-11 \\
4.728 e-11 \\
4.763 e-11 \\
4.778 e-11 \\
4.790 e-11 \\
4.846 e-11 \\
4.951 e-11\end{array}$ & $\begin{array}{l}5.095 e-11 \\
5.093 e-11 \\
5.059 e-11 \\
5.112 e-11 \\
5.196 e-11 \\
5.251 e-11 \\
5.349 e-11 \\
5.416 e-11 \\
5.507 e-11 \\
5.692 e-11 \\
5.832 e-11 \\
5.875 e-11 \\
5.985 e-11 \\
6.067 e-11 \\
6.132 e-11 \\
6.170 e-11 \\
6.211 e-11 \\
6.256 e-11 \\
6.326 e-11 \\
6.387 e-11 \\
6.455 e-11 \\
6.499 e-11 \\
6.516 e-11 \\
6.502 e-11 \\
6.534 e-11 \\
6.626 e-11\end{array}$ & $\begin{array}{l}5.007 e-11 \\
5.024 e-11 \\
5.027 e-11 \\
5.037 e-11 \\
5.113 e-11 \\
5.186 e-11 \\
5.277 e-11 \\
5.343 e-11 \\
5.441 e-11 \\
5.621 e-11 \\
5.754 e-11 \\
5.809 e-11 \\
5.911 e-11 \\
5.991 e-11 \\
6.055 e-11 \\
6.093 e-11 \\
6.133 e-11 \\
6.176 e-11 \\
6.248 e-11 \\
6.313 e-11 \\
6.381 e-11 \\
6.423 e-11 \\
6.440 e-11 \\
6.433 e-11 \\
6.472 e-11 \\
6.567 e-11\end{array}$ \\
\hline
\end{tabular}




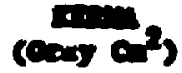

Maste
ans

Inetee

Ineld

masele

Eyudr.

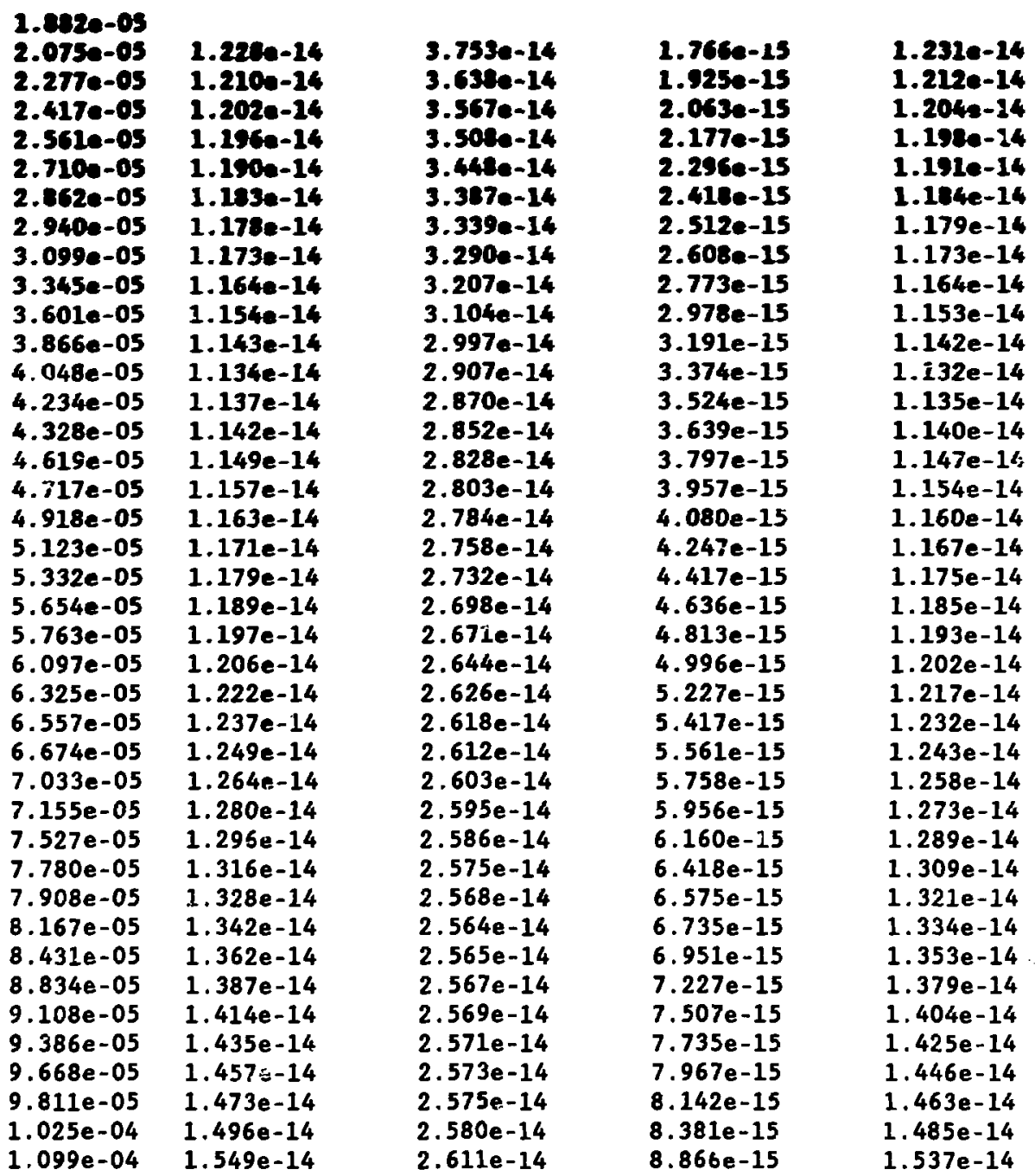




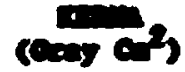

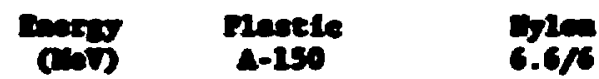

Ineteo

ingerte andelo Epeto.

\begin{tabular}{|c|c|c|c|c|}
\hline $\begin{array}{l}1.251-04 \\
1.339 e-04 \\
1.406 e-04 \\
1.511 e-04 \\
1.601 e-04 \\
1.694 e-04 \\
1.789 e-04 \\
1.037 e-04 \\
1.968 e-04 \\
2.091 e-04 \\
2.741 e-04 \\
3.267 e-04 \\
3.811 e-04 \\
4.704 e-04 \\
4.991 e-04 \\
5.658 e-04 \\
6.043 e-04 \\
6.367 e-04 \\
7.156 e-04 \\
8.322 e-04 \\
9.177 e-04 \\
1.058 e-03 \\
1.307 e-03 \\
1.581 e-03 \\
1.882 e-03 \\
2.208 e-03 \\
2.561 e-03 \\
2.940 e-03 \\
3.345 e-03 \\
3.777 e-03 \\
4.234 e-03 \\
5.763 e-03 \\
7.527 e-03 \\
1.025 e-02 \\
1.511 e-02 \\
2.091 e-02 \\
2.646 e-02 \\
3.267 e-02 \\
3.953 e-02\end{array}$ & $\begin{array}{l}1.761 e-14 \\
1.029 e-14 \\
1.507 e-14 \\
1.995 e-14 \\
2.077 e-14 \\
2.162 e-14 \\
2.249 e-14 \\
2.337 e-14 \\
2.432 e-14 \\
2.801 e-14 \\
3.377 e-14 \\
3.900 e-14 \\
4.608 e-14 \\
5.198 e-14 \\
5.676 e-14 \\
6.202 e-14 \\
6.558 e-14 \\
7.119 e-14 \\
8.103 e-14 \\
9.120 e-14 \\
1.026 e-13 \\
1.222 e-13 \\
1.486 e-13 \\
1.777 e-13 \\
2.093 e-13 \\
2.436 e-12 \\
2.805 e-13 \\
3.199 e-13 \\
3.619 e-13 \\
4.063 e-13 \\
5.049 e-13 \\
6.641 e-13 \\
8.773 e-13 \\
1.224 e-12 \\
1.685 e-12 \\
2.153 e-12 \\
2.617 e-12 \\
3.086 e-12\end{array}$ & $\begin{array}{l}2.079-14 \\
2.739 e-14 \\
2.750 e-14 \\
2.027 e-14 \\
2.080 e-14 \\
2.930 e-14 \\
2.980 e-14 \\
3.033 e-14 \\
3.086 e-14 \\
3.153 e-14 \\
3.466 e-14 \\
3.954 e-14 \\
4.398 e-14 \\
5.016 e-14 \\
5.556 e-14 \\
5.994 e-14 \\
6.476 e-14 \\
6.807 e-14 \\
7.333 e-14 \\
8.255 e-14 \\
9.217 e-14 \\
1.030 e-13 \\
1.217 e-13 \\
1.470 e-13 \\
1.748 e-13 \\
2.052 e-13 \\
2.383 e-13 \\
2.738 e-13 \\
3.118 e-13 \\
3.523 e-13 \\
3.951 e-13 \\
4.903 e-13 \\
6.440 e-13 \\
8.499 e-13 \\
1.185 e-12 \\
1.630 e-12 \\
2.082 e-12 \\
2.528 e-12 \\
2.982 e-12\end{array}$ & $\begin{array}{l}9.490 e-15 \\
1.013 e-14 \\
1.000 e-14 \\
1.142 e-14 \\
1.213 e-14 \\
1.293 e-14 \\
1.367 e-14 \\
1.445 e-14 \\
1.524 e-14 \\
1.605 e-14 \\
1.688 e-14 \\
1.996 e-14 \\
2.477 e-14 \\
2.913 e-14 \\
3.497 e-14 \\
3.978 e-14 \\
4.366 e-14 \\
4.794 e-14 \\
5.083 e-14 \\
5.535 e-14 \\
6.328 e-14 \\
7.146 e-14 \\
8.061 e-14 \\
9.629 e-14 \\
1.174 e-13 \\
1.407 e-13 \\
1.660 e-13 \\
1.934 e-13 \\
2.228 e-13 \\
2.543 e-13 \\
2.878 e-13 \\
3.233 e-13 \\
4.019 e-13 \\
5.289 e-13 \\
6.990 e-13 \\
9.755 e-13 \\
1.343 e-12 \\
1.717 e-12 \\
2.087 e-12 \\
2.463 e-12\end{array}$ & $\begin{array}{l}1.005 e-14 \\
1.675 e-14 \\
1.747 e-14 \\
1.813 e-14 \\
1.050 e-14 \\
1.976 e-14 \\
2.05 e-14 \\
2.141 e-14 \\
2.227 e-14 \\
2.314 e-14 \\
2.407 e-14 \\
2.771 e-14 \\
3.340 e-14 \\
3.856 e-14 \\
4.555 e-14 \\
5.137 e-14 \\
5.610 e-14 \\
6.130 e-14 \\
6.481 e-14 \\
7.036 e-14 \\
8.008 e-14 \\
9.013 e-14 \\
1.014 e-13 \\
1.207 e-13 \\
1.468 e-13 \\
1.755 e-13 \\
2.068 e-13 \\
2.407 e-13 \\
2.771 e-13 \\
3.161 e-13 \\
3.575 e-13 \\
4.014 e-13 \\
4.988 e-13 \\
6.555 e-13 \\
8.653 e-13 \\
1.207 e-12 \\
1.661 e-12 \\
2.121 e-12 \\
2.571 e-12 \\
3.036 e-12\end{array}$ \\
\hline
\end{tabular}




\section{$\cos 2$}

\section{Pant Pasele ans}

\section{Dion}

$6.6 \%$
Incteo

$3.457 e-12$

4.016e-12

4.65se-12

5.337a-12

$6.025 e-12$

$7.028-12$

$0.5230-12$

9.6990-12

1.051e-11

1.132e-11

1.193e-11

$1.263 e-11$

$1.350 \mathrm{e}-11$

$1.442 e-11$

$1.563 e-11$

1.700e-11

$1.879 e-11$

2.028e-11

2.204e-11

2.354e-11

2.504e-11

2.665e-11

$2.789 e-11$

2.941e-11

$3.102 e-11$

$3.197 e-11$

3.328e-11

$3.519 e-11$

$3.823 e-11$

$3.909 e-11$

$4.285 e-11$

$4.408 e-11$

$4.409 e-11$

$4.546 e-11$

$4.518 e-11$

$4.478 e-11$

$4.569 e-11$

$4.607 e-11$

$4.626 e-11$

$4.816 \mathrm{e}-11$ 2.857e-12

3.326e-12

3.064- 12

4.420e-12

4.9940-12

5.033e-12

7.086e-12

$8.077 e-12$

8.768e-12

$9.455 e-12$

$9.983 e-12$

1.058e-11

$1.136 e-11$

$1.229 e-11$

$1.329 e-11$

$1.420 e-11$

$1.565 e-11$

$1.704 e-11$

$1.894 \mathrm{e}-11$

2.004e-11

2.121e-11

$2.208 e-11$

$2.332 e-11$

2.446e-11

2.595e-11

$2.645 e-11$

$2.740 \mathrm{e}-11$

$2.908 e-11$

$3.164 e-11$

3. $236 e-11$

$3.600 e-11$

$3.683 e-11$

$3.632 e-11$

$3.755 e-11$

$3.724 \mathrm{e}-11$

$3.713 e-11$

$3.793 e-11$

$3.816 e-11$

$3.848 e-11$

$3.972 e-11$
Lipuld inselo stoto.

3. 520e-12

4.007e-12

$4.746-12$

5. $422 \mathrm{e}-12$

6.116e-12

7.120e-12

8.640e-12

9.845e-12

1.06Be-11

1.152e-11

1.216e-11

1.290e-11

1.391e-11

$1.529 e-11$

1. 653e-11

1.704e-11

$1.881 e-11$

2.049e-11

2.353e-11

$2.462 e-11$

$2.589 e-11$

2.657e-11

$2.812 e-11$

2.948e-11

$3.090 e-11$

$3.159 e-11$

$3.235 e-11$

$3.430 e-11$

$3.599 e-11$

3.837e-11

4.157e-11

4. $233 e-11$

4. $244 \mathrm{e}-11$

$4.409 e-11$

4. $410 e-11$

$4.438 \mathrm{e}-11$

4. $591 \mathrm{e}-11$

4. $539 e-11$

4.670e-11

4.694e-11 


\section{$(\sin )_{2}$}

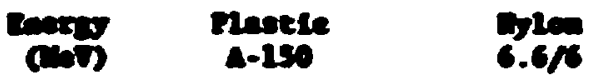

Inelte

Lene

ravele

notro

$6.737 e+00$
$7.156 e+00$
$7.549 e+00$
$7.910 e+00$
$1.322 e+00$
$1.767 e+00$
$9.177 e+00$
$9.655 e+00$
$1.012 e+01$
$1.058 e+01$
$1.101 e+01$
$1.155 e+01$
$1.199 e+01$
$1.250 e+01$
$1.307 e+01$
$1.354 e+01$
$1.386 e+01$
$1.413 e+01$
$1.441 e+01$
$1.468 e+01$
$1.519 e+01$
$1.575 e+01$
$1.633 e+01$
$1.692 e+01$
$1.752 e+01$
$1.813 e+01$
$1.875 e+01$
$2.000 e+01$

4.701e-11

$4.7030-11$

5.174e-11

5.518e-11

5.523e-11

5.310e-11

5.430e-11

5.574e-11

5.625e-11

5.674e-11

$5.877 e-11$

6.032e-11

6.127e-11

6.274e-11

$6.356 e-11$

6.440e-11

6. $495 \mathrm{e}-11$

$6.533 e-11$

6.566e-11

6.603e-11

6.698e-11

6.806e-11

6.897e-11

6.928e-11

6.937e-11

$6.998 e-11$

$7.108 \mathrm{e}-11$

$7.245 e-11$
4.6720-11

4.C010-11

5.0560-11

5.3200-11

5.325e-11

5.150e-11

5.265e-11

5.400e-11

5.466e-11

5.524e-11

5.701e-11

5.858e-11

5.965e-11

6.100e-11

6.195e-11

6.284e-11

6.339e-11

6. $374 \mathrm{e}-11$

6. $410 \mathrm{e}-11$

6. $449 \mathrm{e}-11$

6.534e-11

$6.624 e-11$

$6.702 e-11$

$6.734 \mathrm{e}-11$

$6.743 \mathrm{e}-11$

$6.791 e-11$

6.886e-11

$7.016 e-11$ 3.095e-11

3.097e-11

4.235e-11

4.456e-11

4.450e-11

4.306e-11

4. $4040-11$

$4.517 e-11$

4.575e-11

4.624e-11

4.794e-11

4.966e-11

5.075e-11

5.187e-11

5.274e-11

5.356e-11

$5.415 e-11$

5.454e-11

5.491e-11

5.532e-11

$5.623 \mathrm{e}-11$

5.724e-11

5.815e-11

5.854e-11

5.867e-11

5. $906 e-11$

5.995e-11

6.126e-11
4.020--11

4.05e-11

5.056-11

5.032e-11

5.067e-11

5.111e-11

5.103e-11

5.250e-11

5. 348e-11

5. $417 e-11$

5.506e-11

5.693e-11

5.835e-11

5.881e-11

5.983e-11

6.070e-11

6.136e-11

6.174e-11

6.216e-11

6.261e-11

6.332e-11

$6.394 e-11$

$6.462 e-11$

6.506e-11

6.524e-11

6. $509 e-11$

6.541e-11

$6.634 \mathrm{e}-11$ 


\section{$\cos 28$}

\section{and}

Water acosiden ex Ar

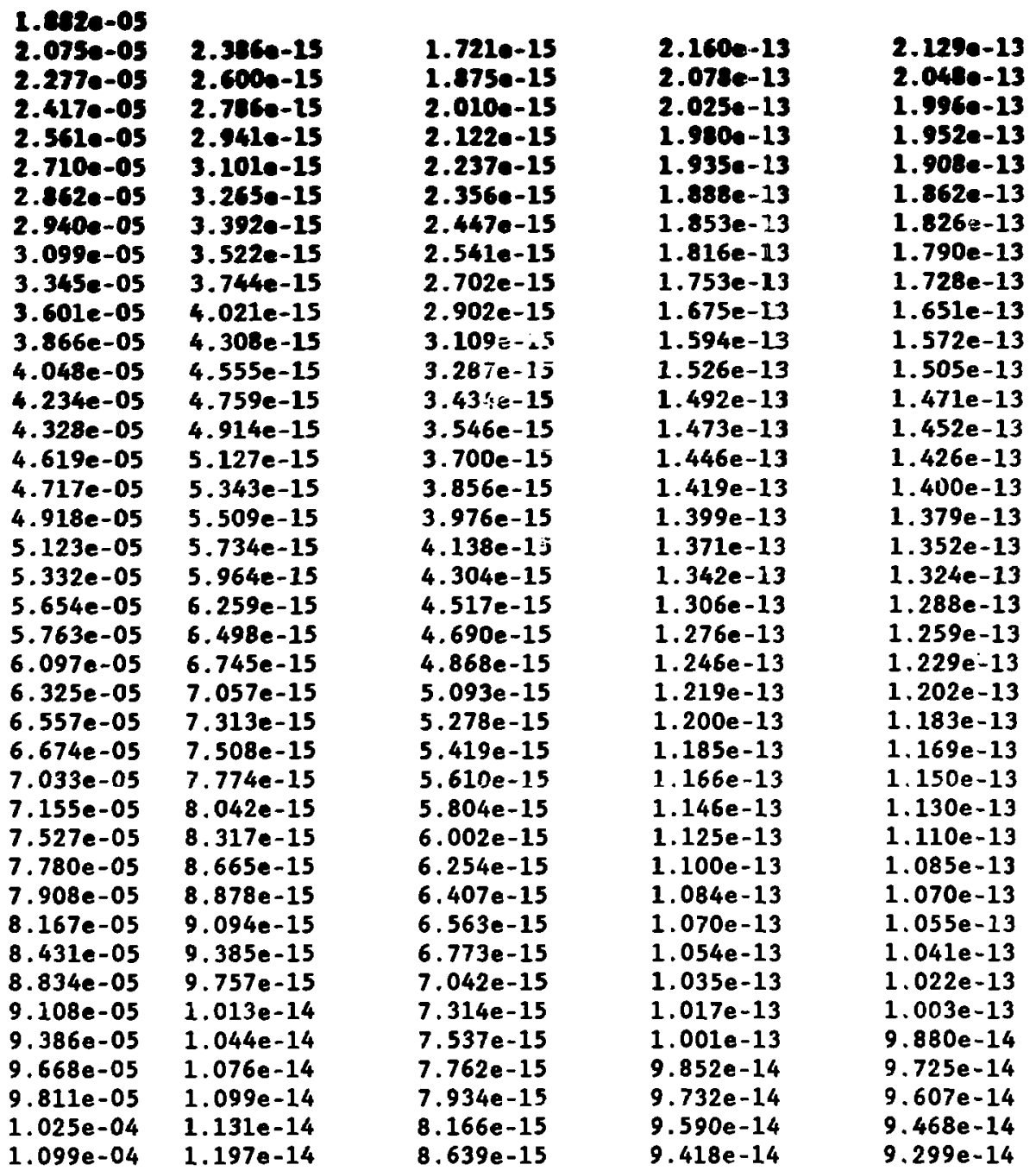




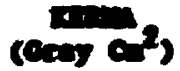

\section{and}

Dees

seotricos

bo ats

Ar.

tater

sac.

$\begin{array}{ll}1.176 e-04 & 1.201 e-14 \\ 1.256 e-04 & 1.365 e-14 \\ 1.339 e-04 & 1.458 e-14 \\ 1.406 e-04 & 1.541 e-14 \\ 1.511 e-04 & 1.637 e-14 \\ 1.601 e-04 & 1.745 e-14 \\ 1.694 e-04 & 1.846 e-14 \\ 1.799 e-04 & 1.950 e-14 \\ 1.887 e-04 & 2.057 e-14 \\ 1.988 e-04 & 2.167 e-14 \\ 2.091 e-04 & 2.279 e-14 \\ 2.741 e-04 & 2.695 e-14 \\ 3.267 e-04 & 3.344 e-14 \\ 3.811 e-04 & 3933 e-14 \\ 4.704 e-04 & 4.722 e-14 \\ 4.991 e-04 & 5.371 e-14 \\ 5.658 e-04 & 5.896 e-14 \\ 6.043 e-04 & 6.474 e-14 \\ 6.367 e-04 & 6.863 e-14 \\ 7.156 e-04 & 7.474 e-14 \\ 8.322 e-04 & 8.546 e-14 \\ 9.177 e-04 & 9.651 e-14 \\ 1.058 e-03 & 1.089 e-13 \\ 1.307 e-03 & 1.301 e-13 \\ 1.581 e-03 & 1.586 e-13 \\ 1.882 e-03 & 1.900 e-13 \\ 2.208 e-03 & 2.242 e-13 \\ 2.561 e-03 & 2.612 e-13 \\ 2.940 e-03 & 3.009 e-13 \\ 3.345 e-03 & 3.434 e-13 \\ 3.777 e-03 & 3.886 e-13 \\ 4.234 e-03 & 4.365 e-13 \\ 5.763 e-03 & 5.426 e-13 \\ 7.527 e-03 & 7.134 e-13 \\ 1.025 e-02 & 9.419 e-13 \\ 1.511 e-02 & 1.315 e-12 \\ 2.091 e-02 & 1.809 e-12 \\ 2.646 e-02 & 2.310 e-12 \\ 3.267 e-02 & 2.798 e-12 \\ 3.953 e-02 & 3.304 e-12\end{array}$

9.246-15

$0.0746-15$

1.052e-14

$1.112 \mathrm{e} .14$

1.101e-14

1.259e-14

1. 332e-14

1. $408 \mathrm{e}-14$

1.485e-14

1.564e-14

1.645e-14

1.945e-14

2. $413 e-14$

2.838e-14

3.407e-14

3.875e-14

4. 254e-14

4.671e-14

4.952e-14

5.392e-14

6.165e-14

$6.961 \mathrm{e}-14$

$7.852 e-14$

$9.379 e-14$

1. $144 \mathrm{e}-13$

$1.370 \mathrm{e}-13$

$1.617 e-13$

$1.884 \mathrm{e}-13$

$2.171 e-13$

$2.477 e-13$

$2.804 e-13$

$3.149 \mathrm{e}-13$

$3.915 e-13$

$5.155 e-13$

$6.816 e-13$

$9.511 \mathrm{e}-13$

1. $310 \mathrm{e}-12$

$1.676 \mathrm{e}-12$

2. 040e-12

$2.407 e-12$
$9.2146-14$

$9.002 e-14$

8.703e-14

$0.560--14$

B.347e-14

c.0046-14

$7.836 e-14$

$7.583 e-14$

7.321e-14

$7.053 e-14$

$6.848 e-14$

6.498e-14

5. $952 e-14$

5.455e-14

4.941e-14

$4.707 \mathrm{e}-14$

4.528e-14

4.330e-14

4. $232 e-14$

4.122e-14

$3.932 e-14$

$3.794 e-14$

$3.679 e-14$

$3.605 e-14$

3. $530 e-14$

$3.445 e-14$

3.387e-14

3. $465 e-14$

$3.558 \mathrm{e}-14$

$3.654 e-14$

3. $756 e-14$

$3.870 e-14$

4. $259 e-14$

4. $934 e-14$

$5.919 e-14$

$7.698 e-14$

$9.952 \mathrm{e}-14$

1. $219 \mathrm{e}-13$

1. $436 \mathrm{e}-13$

$1.669 e-13$ 9.059e-14

$0.8920-14$

$0.677 e-14$

$0.4780-14$

- 250e-14

$7.993 e-14$

$7.750 e-14$

$7.501 e-14$

$7.245 e-14$

$6.982 e-14$

$6.782 e-14$

$6.442 e-14$

5.914e-14

$5.433 e-14$

$4.937 e-14$

$4.716 e-14$

$4.547 e-14$

4. $360 e-14$

$4.269 e-14$

$4.169 e-14$

$3.997 e-14$

$3.877 e-14$

$3.782 \mathrm{e}-14$

$3.739 e-14$

$3.706 e-14$

3.667e-14

3. $659 e-14$

3. $788 \mathrm{e}-14$

$3.937 e-14$

4.093e-14

4. $257 e-14$

4. $439 e-14$

4. $974 e-14$

$5.883 e-14$

$7.181 e-14$

$9.467 e-14$

$1.240 \mathrm{e}-13$

$1.532 e-13$

$1.816 e-13$

2. $118 \mathrm{e}-13$ 


\section{$(\cos 2)$}

\section{0}

water

acesten

Dry atr

Atr.
Datex
sat.

$4.704 e-02$
$5.761 e-02$
$7.002 e-02$
$1.322 e-02$
$9.891 e-02$
$1.307 e-01$
$1.819 e-01$
$2.075 e-01$
$2.417 e-01$
$2.710 e-01$
$2.940 e-01$
$3.345 e-01$
$3.776 e-01$
$4.234 e-01$
$5.123 e-01$
$6.325 e-01$
$7.527 e-01$
$8.834 e-01$
$1.025 e+00$
$1.176 e+00$
$1.338 e+00$
$1.511 e+00$
$1.694 e+00$
$1.887 e+00$
$2.091 e+00$
$2.305 e+00$
$2.530 e+00$
$2.741 e+00$
$3.011 e+00$
$3.267 e+00$
$3.534 e+00$
$3.811 e+00$
$4.069 e+00$
$4.396 e+00$
$4.704 e+00$
$4.991 e+00$
$5.352 e+00$
$5.658 e+00$
$6.042 e+00$
$6.367 e+00$

$3.8320-12$

4.447e-12

5.166e-12

5.8se -12

6.6500-12

$7.747 a-12$

$9.386 a-12$

1.069e-11

1.160e-11

1.251e-11

1. 321e-11

1.401e-11

1.512e-11

$1.668 e-11$

1. 801e-11

1.845e-11

2.033e-11

2.220e-11

2.567e-11

2.679e-11

2.812e-11

$2.866 e-11$

3.042e-11

3.184e-11

3. $334 e-11$

3. 406e-11

$3.479 \mathrm{e}-11$

$3.683 \mathrm{e}-11$

$3.828 e-11$

4.110e-11

4. $424 \mathrm{e}-11$

$4.496 e-11$

$4.513 e-11$

4.691e-11

4. $710 \mathrm{e}-11$

$4.765 e-11$

$4.938 e-11$

$4.873 e-11$

$5.038 e-11$

5. 024e-11

$2.791 e-12$
$3.246 e-12$
$3.760 e-12$
$4.328 e-12$
$4.095 e-12$
$5.723 e-12$
$6.957 e-12$
$7.925 e-12$
$8.598 e-12$
$9.264 e-12$
$9.773 e-12$
$1.035 e-11$
$1.105 e-11$
$1.173 e-11$
$1.269 e-11$
$1.407 e-11$
$1.550 e-11$
$1.681 e-11$
$1.803 e-11$
$1.932 e-11$
$2.060 e-11$
$2.183 e-11$
$2.298 e-11$
$2.415 e-11$
$2.580 e-11$
$2.634 e-11$
$2.760 e-11$
$2.923 e-11$
$3.252 e-11$
$3.225 e-11$
$3.626 e-11$
$3.730 e-11$
$3.655 e-11$
$3.760 e-11$
$3.715 e-11$
$3.680 e-11$
$3.718 e-11$
$3.800 e-11$
$3.768 e-11$
$3.977 e-11$

1.914- -13

2.217e-13

2.56e- -13

2.913e-13

3.2590-13

3.849-13

4.8910-13

$5.897 e-13$

6.614e-13

7.315e-13

7.847e-13

8.513e-13

$9.766 e-13$

1. $228 \mathrm{e}-12$

1. 603e-12

1.100e-12

$1.827 e-12$

1. $346 e-12$

1. $798 e-12$

2.023e-12

2.186e-12

3. $834 \mathrm{e}-12$

2.831e-12

3. $711 e-12$

$3.130 \mathrm{e}-12$

3.801e-12

3. $720 \mathrm{e}-12$

$5.009 e-12$

6. 504e-12

8. 682e-12

$1.051 \mathrm{e}-11$

1.131e-11

1. 269e-11

1. 365e-11

1. 201e-11

$9.205 e-12$

$1.052 e-11$

8. $544 \mathrm{e}-12$

$8.612 e-12$

$1.030 e-11$
2.4350-13

2.8210-13

3.2700-13

3.715e-13

4.163e-13

4.902e-13

6.163e-13

7.342e-13

$8.178 e-13$

8.999e-13

$9.625 e-13$

1.039e-12

1.179e-12

$1.449 e-12$

1. 837e-12

1. $348 \mathrm{e}-12$

2.091e-12

$1.644 \mathrm{e}-12$

2.140e-12

$2.377 e-12$

$2.557 e-12$

4.189e-12

3. 226e-12

4. $113 e-12$

3. $562 e-12$

4. $234 e-12$

4. $164 \mathrm{e}-12$

$5.464 e-12$

$6.958 \mathrm{e}-12$

$9.145 e-12$

$1.099 e-11$

$1.179 e-11$

1. $316 e-11$

$1.412 \mathrm{e}-11$

$1.251 e-11$

$9.754 \mathrm{e}-12$

1. $107 e-11$

$9.118 e-12$

$9.209 e-12$

$1.087 e-11$ 


\section{$\cos 2)$}

\begin{tabular}{|c|c|c|c|}
\hline & water & seatglane & trs \\
\hline
\end{tabular}

\begin{tabular}{|c|c|c|c|c|}
\hline $\begin{array}{l}1.737 e+00 \\
7.256 e+00 \\
7.546 e+00 \\
7.910 e+00 \\
.322 e+00 \\
.767 e+00 \\
.177 e+00 \\
.655 e+00 \\
1.012 e+01 \\
1.058 e+01 \\
1.101 e+01 \\
1.155 e+01 \\
1.199 e+01 \\
1.250 e+01 \\
1.307 e+01 \\
1.354 e+01 \\
1.386 e+01 \\
1.413 e+01 \\
1.441 e+01 \\
1.468 e+01 \\
1.519 e+01 \\
1.575 e+01 \\
1.633 e+01 \\
1.692 e+01 \\
1.752 e+01 \\
1.813 e+01 \\
1.875 e+01 \\
2.000 e+01\end{array}$ & $\begin{array}{l}5.233 e-11 \\
5.205 e-11 \\
5.491 e-11 \\
5.405 e-11 \\
5.397 e-11 \\
5.459 e-11 \\
5.552 e-11 \\
5.605 e-11 \\
5.712 e-11 \\
5.786 e-11 \\
5.855 e-11 \\
6.052 e-11 \\
6.201 e-11 \\
6.225 e-11 \\
6.336 e-11 \\
6.416 e-11 \\
6.486 e-11 \\
6.525 e-11 \\
6.569 e-11 \\
6.615 e-11 \\
6.683 e-11 \\
6.739 e-11 \\
6.806 e-11 \\
6.854 e-11 \\
6.873 e-11 \\
6.842 e-11 \\
6.858 e-11 \\
6.941 e-11\end{array}$ & $\begin{array}{l}3.756 e-11 \\
3.734 e-11 \\
4.152 e-11 \\
4.556 e-11 \\
4.547 e-11 \\
4.276 e-11 \\
4.397 e-11 \\
4.547 e-11 \\
4.583 e-11 \\
4.622 e-11 \\
4.848 e-11 \\
5.006 e-11 \\
5.095 e-11 \\
5.255 e-11 \\
5.331 e-11 \\
5.415 e-11 \\
5.468 e-11 \\
5.508 e-11 \\
5.540 e-11 \\
5.578 e-11 \\
5.682 e-11 \\
5.806 e-11 \\
5.910 e-11 \\
5.943 e-11 \\
5.951 e-11 \\
6.027 e-11 \\
6.155 e-11 \\
6.309 e-11\end{array}$ & $\begin{array}{l}6.366 e-12 \\
1.447 e-12 \\
1.134 e-11 \\
1.017 e-11 \\
1.026 e-11 \\
9.499 e-12 \\
1.031 e-11 \\
1.115 e-11 \\
1.230 e-11 \\
1.312 e-11 \\
1.346 e-11 \\
1.461 e-11 \\
1.597 e-11 \\
1.699 e-11 \\
1.846 e-11 \\
1.956 e-11 \\
1.998 e-11 \\
2.008 e-11 \\
2.051 e-11 \\
2.098 e-11 \\
2.116 e-11 \\
2.104 e-11 \\
2.089 e-11 \\
2.109 e-11 \\
2.124 e-11 \\
2.125 e-11 \\
2.168 e-11 \\
2.277 e-11\end{array}$ & $\begin{array}{l}6.995 e-12 \\
9.092 e-12 \\
1.196 e-11 \\
1.079 e-11 \\
1.096 e-11 \\
1.015 e-11 \\
1.095 e-11 \\
1.279 e-11 \\
1.294 e-11 \\
1.375 e-11 \\
1.411 e-11 \\
1.527 e-11 \\
1.663 e-11 \\
1.763 e-11 \\
1.910 e-11 \\
2.020 e-11 \\
2.062 e-11 \\
2.072 e-11 \\
2.116 e-11 \\
2.163 e-11 \\
2.182 e-11 \\
2.170 e-11 \\
2.156 e-11 \\
2.177 e-11 \\
2.192 e-11 \\
2.192 e-11 \\
2.235 e-11 \\
2.344 e-11\end{array}$ \\
\hline
\end{tabular}




\section{$(0 x)$ a}

\section{4)} ans
Eesplamo

\section{provile}

Cas. To. - Minethasa

$\begin{array}{llll}1.002 e-05 & & & \\ 2.075 e-05 & 6.560 e-17 & 3.102 e-15 & 1.227 e-14 \\ 2.277 e-05 & 7.203 e-17 & 3.379 e-15 & 1.209 e-14 \\ 2.417 e-05 & 7.760 e-17 & 3.621 e-15 & 1.201 e-14 \\ 2.561 e-05 & \mathbf{2 0 2 3 e - 1 7} & 3.822 e-15 & 1.195 e-14 \\ 2.710 e-05 & \mathbf{6 9 9}-17 & 4.030 e-15 & 1.128 e-14 \\ 2.862 e-05 & 9.199 e-17 & 4.244 e-15 & 1.181 e-14 \\ 2.940 e-05 & 9.563 e-17 & 4.408 e-15 & 1.177 e-14 \\ 3.099 e-05 & 9.949 e-17 & 4.577 e-15 & 1.171 e-14 \\ 3.345 e-05 & 1.061 e-16 & 4.867 e-15 & 1.163 e-14 \\ 3.691 e-05 & 1.142 e-16 & 5.226 e-15 & 1.152 e-14 \\ 3.866 e-05 & 1.227 e-16 & 5.599 e-15 & 1.141 e-14 \\ 4.048 e-05 & 1.300 e-16 & 5.920 e-15 & 1.132 e-14 \\ 4.234 e-05 & 1.360 e-16 & 6.185 e-15 & 1.135 e-14 \\ 4.328 e-05 & 1.405 e-16 & 6.386 e-15 & 1.140 e-14 \\ 4.619 e-05 & 1.468 e-16 & 6.663 e-15 & 1.147 e-14 \\ 4.717 e-05 & 1.531 e-16 & 6.944 e-15 & 1.155 e-14 \\ 4.918 e-05 & 1.580 e-16 & 7.160 e-15 & 1.160 e-14 \\ 5.123 e-05 & 1.646 e-16 & 7.452 e-15 & 1.168 e-14 \\ 5.332 e-05 & 1.713 e-16 & 7.7513-15 & 1.176 e-14 \\ 5.654 e-05 & 1.799 e-16 & 8.134 e-15 & 1.186 e-14 \\ 5.763 e-05 & 1.869 e-16 & 8.445 e-15 & 1.195 e-14 \\ 6.097 e-05 & 1.941 e-16 & 8.765 e-15 & 1.204 e-14 \\ 6.325 e-05 & 2.033 e-16 & 9.171 e-15 & 1.219 e-14 \\ 6.557 e-05 & 2.108 e-16 & 9.504 e-15 & 1.234 e-14 \\ 6.674 e-05 & 2.164 e-16 & 9.757 e-15 & 1.246 e-14 \\ 7.033 e-05 & 2.242 e-16 & 1.010 e-14 & 1.261 e-14 \\ 7.155 e-05 & 2.320 e-16 & 1.045 e-14 & 1.276 e-14 \\ 7.527 e-05 & 2.400 e-16 & 1.081 e-14 & 1.292 e-14 \\ 7.780 e-05 & 2.502 e-16 & 1.126 e-14 & 1.312 e-14 \\ 7.908 e-05 & 2.564 e-16 & 1.154 e-14 & 1.325 e-14 \\ 8.167 e-05 & 2.627 e-16 & 1.182 e-14 & 1.338 e-14 \\ 8.431 e-05 & 2.712 e-16 & 1.220 e-14 & 1.358 e-14 \\ 8.034 e-05 & 2.820 e-16 & 1.268 e-14 & 1.383 e-14 \\ 9.108 e-05 & 2.930 e-16 & 1.317 e-14 & 1.409 e-14 \\ 9.386 e-05 & 3.020 e-16 & 1.357 e-14 & 1.431 e-14 \\ 9.668 e-05 & 3.111 e-16 & 1.398 e-14 & 1.452 e-14 \\ 9.811 e-05 & 3.180 e-16 & 1.428 e-14 & 1.468 e-14 \\ 1.025 e-04 & 3.273 e-16 & 1.470 e-14 & 1.492 e-14 \\ 1.099 e-04 & 3.465 e-16 & 1.556 e-14 & 1.544 e-14\end{array}$




\section{$(\cos 2)$}

\section{5 \\ (ㄷ)}

Carton

Diande
Ielysiean

$1.665 e-14$

1.778e-14

1.895e-14

2.003e-14

2.127e-14

2.267e-14

2.399e-14

2.534e-14

$2.673 e-14$

2.815e-14

2.961e-14

3.501e-14

4. $344 e-14$

5.109e-14

$6.135 e-14$

$6.977 e-14$

$7.659 e-14$

8. $410 e-14$

$8.915 e-14$

$9.708 e-14$

$1.110 e-13$

$1.253 e-13$

$1.414 e-13$

$1.689 e-13$

$2.059 e-13$

$2.467 e-13$

2. 91 ie-13

$3.392 e-13$

$3.908 e-13$

$4.460 e-13$

$5.047 e-13$

$5.670 \mathrm{e}-13$

$7.048 e-13$

$9.272 \mathrm{e}-13$

$1.225 e-12$

$1.709 \mathrm{e}-12$

2. $352 e-12$

$3.005 e-12$

$3.648 e-12$

4. $304 e-12$
Cas. Te. - Minethaso
$1.612 e-14$

$1.682 e-14$

1.755e-14

$1.823 \mathrm{e}-14$

$1.900 e-14$

1.987e-14

2.070e-14

2.154e-14

2. 240e-14

$2.329 e-14$

2.423e-14

2.790e-14

$3.364 e-14$

$3.884 e-14$

$4.589 e-14$

$5.176 e-14$

$5.652 e-14$

6.177e-14

$6.531 e-14$

$7.090 \mathrm{e}-14$

8.070e-14

$9.083 e-14$

1. $022 e-13$

$1.217 e-13$

$1.480 \mathrm{e}-13$

$1.769 e-13$

$2.085 e-13$

$2.426 e-13$

$2.793 e-13$

$3.186 e-13$

$3.604 \mathrm{e}-13$

4.047e-13

$5.028 e-13$

6.611e-13

8.730e-13

$1.218 \mathrm{e}-12$

1. $676 \mathrm{e}-12$

2. $141 \mathrm{e}-12$

2.598e-12

3. 067e-12 


\title{
$\left(\cos c^{2}\right)$
}

\section{Inest \\ (10)}

\begin{abstract}
carton
Diorte
\end{abstract}

Eetry leave

\section{1. $395 e-13$}

1. 607e-13

2.057e-13

$2.470 \mathrm{e}-13$

2.936e-13

$3.678 e-13$

4.937e-13

6.176e-13

$7.207 e-13$

8.343e-13

9.298e-13

1. $049 e-12$

1. 311e-12

1. 948 e-12

2.146e-12

1.252e-12

$1.517 \mathrm{e}-12$

1. 824e-12

$3.441 e-12$

$3.134 \mathrm{e}-12$

3. 032e-12

2. 297e-12

2.577e-12

2. $628 \mathrm{e}-12$

$2.935 e-12$

2. $308 \mathrm{e}-12$

$1.928 e-12$

2.817e-12

$3.705 e-12$

$4.638 \mathrm{e}-12$

7. 231e-12

7.008 e -12

$5.914 \mathrm{e}-12$

$6.679 e-12$

$5.666 e-12$

$5.177 e-12$

$5.871 e-12$

$4.778 \mathrm{e}-12$

$5.293 e-12$

5. 091e-12
4. $9500-12$
5. $795 a-12$
6.735e-12
$7.696 e-12$
$8.685 e-12$
1. 012e-11
1.226e-11
1. 393e-11
$1.509 e-11$
1. $622 \mathrm{e}-11$
$1.709 e-11$
$1.807 e-11$
$1.925 e-11$
2.041e-11
2.205e-11
2. 436e-11
2. 675e-11
$2.898 e-11$
$3.112 e-11$
3. 331e-11
3. $549 e-11$
3. $760 e-11$
$3.960 e-11$
$4.160 \mathrm{e}-11$
$4.402 e-11$
4. $539 e-11$
4. 741e-11
4.971e-11
5. $356 e-11$
$5.412 e-11$
$5.858 \mathrm{e}-11$
$6.024 \mathrm{e}-11$
$6.019 \mathrm{e}-11$
$6.179 e-11$
$6.202 \mathrm{e}-11$
$6.222 e-11$
$6.310 e-11$
$6.436 e-11$
$6.453 \mathrm{e}-11$
6.691e-11

ces. 2e. 


\section{$\left.\cos a^{2}\right)$}

\begin{tabular}{|c|c|}
\hline $\begin{array}{l}\cos 28 y \\
\operatorname{arv} 5\end{array}$ & $\begin{array}{l}\text { Carbod } \\
\text { plortde }\end{array}$ \\
\hline
\end{tabular}

$\begin{array}{llll}6.737 e+00 & 5.562 e-12 & 6.523 e-11 & 4.810 e-11 \\ 7.156 e+00 & 5.305 e-12 & 6.541 e-11 & 4.830 e-11 \\ 7.548 e+00 & 7.565 e-12 & 6.966 e-11 & 5.145 e-11 \\ 7.910 e+00 & 7.662 e-12 & 7.371 e-11 & 5.311 e-11 \\ 8.322 e+00 & 7.059 e-12 & 7.390 e-11 & 5.304 e-11 \\ 8.787 e+00 & 6.625 e-12 & 7.167 e-11 & 5.217 e-11 \\ 9.177 e+00 & 7.090 e-12 & 7.305 e-11 & 5.314 e-11 \\ 9.665 e+00 & 7.567 e-12 & 7.469 e-11 & 5.421 e-11 \\ 1.012 e+01 & 8.171 e-12 & 7.526 e-11 & 5.496 e-11 \\ 1.058 e+01 & 8.579 e-12 & 7.582 e-11 & 5.555 e-11 \\ 1.101 e+01 & 9.564 e-12 & 7.808 e-11 & 5.703 e-11 \\ 1.155 e+01 & 1.140 e-11 & 7.969 e-11 & 5.877 e-11 \\ 1.199 e+01 & 1.267 e-11 & 8.066 e-11 & 5.996 e-11 \\ 1.250 e+01 & 1.316 e-11 & 8.226 e-11 & 6.094 e-11 \\ 1.307 e+01 & 1.410 e-11 & 8.308 e-11 & 6.189 e-11 \\ 1.354 e+01 & 1.487 e-11 & 8.394 e-11 & 6.273 e-11 \\ 1.386 e+01 & 1.548 e-11 & 8.451 e-11 & 6.334 e-11 \\ 1.413 e+01 & 1.584 e-11 & 8.493 e-11 & 6.372 e-11 \\ 1.441 e+01 & 1.626 e-11 & 6.525 e-11 & 6.410 e-11 \\ 1.468 e+01 & 1.674 e-11 & 8.561 e-11 & 6.452 e-11 \\ 1.519 e+01 & 1.753 e-11 & 8.662 e-11 & 6.535 e-11 \\ 1.575 e+01 & 1.834 e-11 & 8.778 e-11 & 6.621 e-11 \\ 1.633 e+01 & 1.916 e-11 & 8.877 e-11 & 6.702 e-11 \\ 1.692 e+01 & 1.968 e-11 & 8.906 e-11 & 6.741 e-11 \\ 1.752 e+01 & 1.988 e-11 & 8.912 e-11 & 6.754 e-11 \\ 1.813 e+01 & 1.991 e-11 & 8.979 e-11 & 6.777 e-11 \\ 1.875 e+01 & 2.049 e-11 & 9.093 e-11 & 6.849 e-11 \\ 2.000 e+01 & 2.176 e-11 & 9.228 e-11 & 6.965 e-11\end{array}$




\section{$\cos \cos ^{2}$}

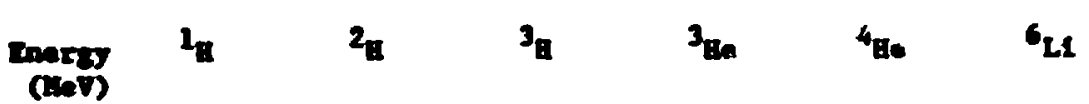

\section{$1.307 e-09$}

5.227e-09 $1.232 e-13 \quad 5.448 e-16 \quad 5.101 e-20 \quad 5.695 e-07 \quad 1.848 e-20 \quad 2.427 e-07$ 2.091e-08 $6.159 e-14 \quad 2.729 e-16 \quad 2.041 e-19 \quad 2.850 e-07 \quad 7.393 e-20 \quad 1.214 e-07$ 3.267e-08 4.108e-14 1.842e-16 4.183e-1s :.912e-07 1.516e-19 8.092e-08 4.704e-08 3.363e-14 $1.523 e-16 \quad 6.224 e-19 \quad 1.569 e-07 \quad 2.255 e-19 \quad 6.621 e-08$ 8.322e-08 2.649e-14 $1.224 e-16 \quad 1.017 e-18 \quad 1.235 e-07 \quad 3.685 e-19 \quad 5.209 e-08$ $1.307 e-072.065 e$ ? $9.897 e=171.670 e-189.590 e-086 . n^{\prime}=19 \quad 4.050 e-08$ 1.882e-07 1.695e-14 8.633e-17 2.489e-18 7.856e-08 9.020e-19 3.310e-08 2 561e-07 $1.442 e-14 \quad 7.946 e-17 \quad 3.469 e-18 \quad 6.645 e-08 \quad 1.257 e-18 \quad 2.801 e-08$

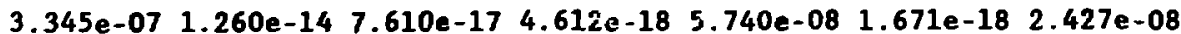
4.234e-07 $1.123 e-14 \quad 7.590 e-17 \quad 5.918 e-18 \quad 5.084 e-08 \quad 2.144 a-18 \quad 2.142 e-03$ 5.123e-07 $1.023 e-14 \quad 7.736 e-17 \quad 7.365 e-18 \quad 4.561 e-08 \quad 2.647 e-18 \quad 1.926 e-08$ 7.527e-07 9.053e-15 $8.341 e-17 \quad 9.877 e-18 \quad 3.939 e-08 \quad 3.578 e-18 \quad 1.662 e-08$ $\begin{array}{lllllll}1.176 e-06 & 7.793 e-15 & 1.003 e-16 & 1.506 e-17 & 3.198 e-08 & 5.456 e-18 & 1.349 e-08\end{array}$ $1.511 e-06 \quad 7.095 e-15 \quad 1.230 e-16 \quad 2.098 e-17 \quad 2.694 e-08 \quad 7.600 e-18 \quad 1.138 e-08$ $2.091 e-06 \quad 6.763 e-15 \quad 1.527 e-16 \quad 2.812 e-17 \quad 2.337 e-08 \quad 1.019 e-17 \quad 9.843 e-09$ $\begin{array}{lllllll}2.741 e-06 & 6.681 e-15 & 1.942 e-16 & 3.773 e-17 & 2.011 e-08 & 1.367 e-17 & 8.489 e-09\end{array}$ 3.534e-06 $6.862 e-15 \quad 2.440 e-16 \quad 4.899 e-17 \quad 1.761 e-08 \quad 1.775 e-17 \quad 7.447 e-09$ $\begin{array}{lllllll}4.704 e-06 & 7.347 e-15 & 3.130 e-16 & 6.432 e-17 & 1.545 e-08 & 2.330 e-17 & 6.503 e-09\end{array}$ $\begin{array}{llllllll}5.658 e-06 & 8.029 e-15 & 3.883 e-16 & 8.091 e-17 & 1.368 e-08 & 2.931 e-17 & 5.789 e-09\end{array}$ 6.737e-05 $8.775 e-15 \quad 4.608 e-16 \quad 9.677 e-17 \quad 1.260 e-08 \quad 3.506 e-17 \quad 5.292 e-09$ 8.321e-06 9.835e-15 5.562e-16 $1.176 e-16 \quad 1.134 e-08 \quad 4.260 e-17 \quad 4.804 e-09$ 9.620e-06 1.104e-14 6.500e-16 $1.401 e-16 \quad 1.047 e-08 \quad 5.075 e-17 \quad 4.397 e-09$ $1.101 e-051.222 e-14 \quad 7.571 e-16 \quad 1.611 e-16 \quad 9.690 e-09 \quad 5.836 e-17 \quad 4.100 e-09$

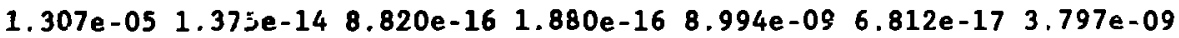

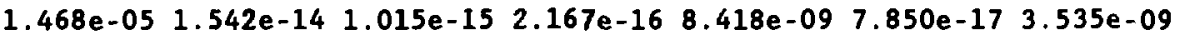
$1.581 e-05 \quad 1.669 e-14 \quad 1.114 e-15 \quad 2.381 e-16 \quad 7.987 e-098.626 e-17 \quad 3.371 e-09$ $\begin{array}{lllllll}1.758 e-05 & 1.804 e-14 & 1.219 e-15 & 2.607 e-16 & 7.588 e-09 & 9.447 e-17 & 3.222 e-09\end{array}$

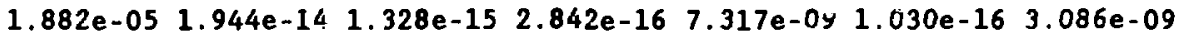
$2.075 e-05 \quad 2.093 e-14 \quad 1.443 e-15 \quad 3.089 e-16 \quad 7.047 e-09 \quad 1.119 e-16 \quad 2.960 e-09$ $2.277 e-05 \quad 2.280 e-1.4 \quad 1.586 e-15 \quad 3.397 e-16 \quad 6.710 e-09 \quad 1.231 e-16 \quad 2.822 e-09$ $2.417 e-05 \quad 2.443 e-14 \quad 1.710 e-15 \quad 3.665 e-16 \quad 6.418 e-09 \quad 1.328 e-16 \quad 2.717 e-09$ $2.561 e-05 \quad 2.579 e-14 \quad 1.813 e-15 \quad 3.887 e-16 \quad 6.203 e-09 \quad 1.408 e-16 \quad 2.638 e-09$ $2.710 e-05 \quad 2.719 e-14 \quad 1.919 e-15 \quad 4.115 e-16 \quad 6.062 e-09 \quad 1.491 e-16 \quad 2.564 e-09$ $\begin{array}{lllllll}2.862 e-05 & 2.863 e-14 & 2.028 e-15 & 4.350 e-16 & 5.923 e-09 & 1.576 e-16 & 2.494 e-09\end{array}$ $2.940 e-05 \quad 2.973 e-14 \quad 2.111 e-15 \quad 4.530 e-16 \quad 5.316 e-09 \quad 1.641 e-16 \quad 2.444 e-09$ $3.099 e-05 \quad 3.087 e-14 \quad 2.197 e-15 \quad 4.715 e-16 \quad 5.706 e-09 \quad 1.708 e-16 \quad 2.395 e-09$ 3.345e-05 3.282e-14 2.344e-15 5.032 a-16 5.518e-09 $1.823 e-16 \quad 2.319 e-09$ $\begin{array}{lllllll}3.601 e-05 & 3.525 e-14 & 2.525 e-15 & 5.423 e-16 & 5.285 e-09 & 1.965 e-16 & 2.234 e-09\end{array}$ $3.866 e-05 \quad 3.776 e-142.714 e-15 \quad 5.830 e-16 \quad 5.069 e-092.112 e-16 \quad 2.154 e-09$ $4.048 e-05 \quad 3.992 e-14 \quad 2.876 e-15 \quad 6.179 e-16 \quad 4.943 e-09 \quad 2.239 e-16 \quad 2.092 e-09$ 

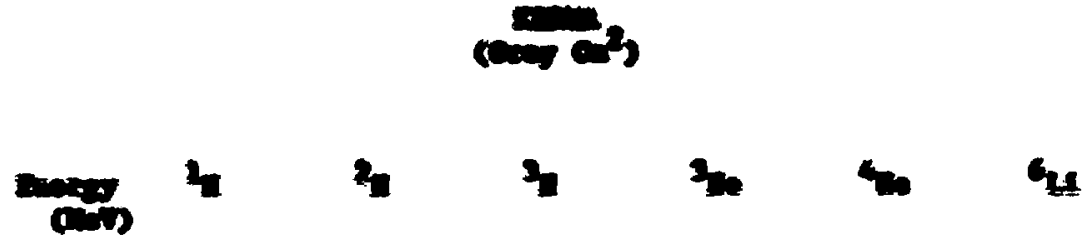

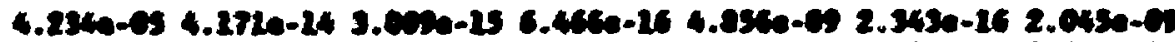

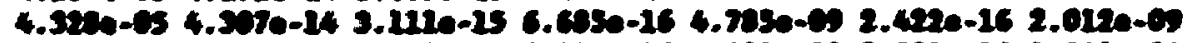

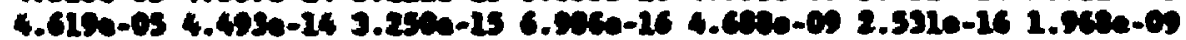

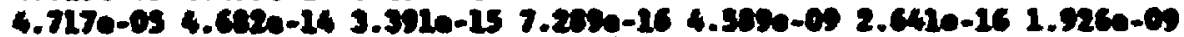

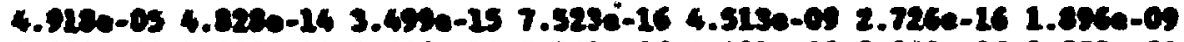
5.1230-05 5.025e-14 3.646-15 7.640e-16 4.4210-6s 2.040e-16 1.054a-cs

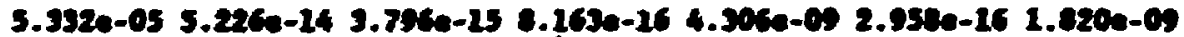
5.654e-05 5.435e-14 3.90ee-15 e.577e-16 4.177e-09 3.104e-16 $1.776 e-09$ $5.763 e-055.6940-14$ 4.144e-15 e.913e-16 4.104e-0s $3.229 e-161.742 e-09$ $6.097 e-055.9100-14$ 4.305e-15 5.260e-16 4.055e-0s 3.355e-16 1.705e-0s $6.325 e-05$ 6.214e-14 4.502e-15 9.631e-16 3.939e-09 3.514e-16 1.670e-09 $6.557=-05$ 6.406e-14 4.675e-15 1.006e-15 3.934e-0s 3.644e-16 1.640e-09 $6.674 e-05$ 6.579e-14 4.801e-15 $1.033 e-15$ 3.092e-09 $3.743 e-161.618 e-09$ $7.033 e-05$ 6.812e-14 4.974e-15 $1.070 e-15$ 3.836e-09 3.878e-16 1.590e-09 7.155e-05 7.046e-14 5.148e-15 1.108e-15 3.779e-09 4.014e-16 1.562e-09 $.7 .527 e-05 \quad 7.287 e-14 \quad 5.327 e-15$ 1.146e-15 3.720e-09 4.153e-16 1.536e-09 $7.780 e-05 \quad 7.592 e-14 \quad 5.553 e-15$ 1.195e-15 $3.646 e-094.330 e-16 \quad 1.504 e-09$ 7.908e-05 $7.779 e-14 \quad 5.691 e-15 \quad 1.225 e-15 \quad 3.601 e-09 \quad 4.438 e-16 \quad 1.486 e-09$ $8.167 e-05 \quad 7.968 e-14 \quad 5.831 e-15 \quad 1.255 e-15 \quad 3.554 e-09 \quad 4.548 e-16 \quad 1.468 e-09$ $8.431 e-05 \quad 8.223 e-14 \quad 6.021 e-15$ 1.296e-15 $3.492 e-09 \quad 4.695 e-16 \quad 1.445 e-09$ $8.834 e-05 \quad 8.549 e-14 \quad 6.262 e-15 \quad 1.348 e-15 \quad 3.413 e-09 \quad 4.884 e-16 \quad 1.416 e-09$ $9.108 e-05 \quad 8.080 e-14 \quad 6.507 e-15 \quad 1.401 e-15 \quad 3.333 e-09 \quad 5.075 e-16 \quad 1.389 e-09$ 9.386e-05 9.150e-14 6.707e-15 $1.444 e-15 \quad 3.267 e-09 \quad 5.232 e-16 \quad 1.369 e-09$ $9.668 e-05 \quad 9.424 e-14 \quad 6.910 e-15 \quad 1.488 e-15 \quad 3.201 e-09 \quad 5.390 e-16 \quad 1.348 e-09$ 9.811e-05 9.632e-14 7.064e-15 $1.521 e-15$ 3.150e-09 $5.510 e-16 \quad 1.334 e-09$ $1.025 e-049.914 e-14 \quad 7.273 e-15 \quad 1.566 e-15 \quad 3.089 e-09 \quad 5.673 e-16 \quad 1.314 e-09$ $1.099 e-041.049 e-13 \quad 7.700 e-15 \quad 1.658 e-15 \quad 3.006 e-09 \quad 6.007 e-16 \quad 1.278 e-09$ 1.176e-04 $1.123 e-13 \quad 8.249 e-15 \quad 1.776 e-15 \quad 2.906 e-09 \quad 6.436 e-16 \quad 1.235 e-09$ $1.256 e-041.199 e-13 \quad 8.816 e-15 \quad 1.899 e-15 \quad 2.802 e-09 \quad 6.880 e-16 \quad 1.194 e-09$ $1.338 e-64 \quad 1.277 e-13 \quad 9.403 e-15 \quad 2.0<5 e-15 \quad 2.719 e-09 \quad 7.338 e-16 \quad 1.157 e-09$ 1.406e-04 $1.350 e-13 \quad 9.947 e-15 \quad 2.142 e-15 \quad 2.658 e-09 \quad 7.762 e-16 \quad 1.124 e-09$ 1.511e-04 $1.434 e-13 \quad 1.057 e-14 \quad 2.277 e-15 \quad 2.588 e-09 \quad 8.250 e-16 \quad 1.090 e-09$ $1.601 e-04 \quad 1.529 e-13 \quad 1.128 e-14 \quad 2.429 e-15 \quad 2.508 e-09 \quad 8.802 e-16 \quad 1.056 e-09$ $1.694 e-04 \quad 1.617 e-13 \quad 1.194 e-14 \quad 2.572 e-15 \quad 2.434 e-09 \quad 9.319 e-16 \quad 1.026 e-09$ $1.789 e-04 \quad 1.708 e-13 \quad 1.262 e-14 \quad 2.719 e-15 \quad 2.357 e-09 \quad 9.852 e-16 \quad 9.979 e-10$ $1.887 e-04 \quad 1.802 e-13 \quad 1.332 e-14 \quad 2.870 e-15 \quad 2.279 e-09 \quad 1.040 e-15 \quad 9.710 e-1 n$ $1.988 e-04 \quad 1.898 e-13 \quad 1.404 e-14 \quad 3.025 e-15 \quad 2.222 e-09 \quad 1.096 e-15 \quad 9.460 e-10$ 2.091e-04 1.996e-13 1.478e-14 3.184e-15 2.176e-09 $1.154 e-15 \quad 9.220 e-10$ 2.741e-04 2.360e-13 $1.751 e-14 \quad 3.773 e-15 \quad 2.006 e-09 \quad 1.367 e-15 \quad 8.489 e-10$ $3.267 e-04 \quad 2.929 e-13 \quad 2.177 e-14 \quad 4.691 e-15 \quad 1.785 e-09 \quad 1.700 e-15 \quad 7.603 e-10$ 3.811e-04 3.445e-13 2.564e-14 5.526e-15 $1.656 e-092.002 e-15 \quad 7.003 e-10$ 


\title{
$\cos a$
}

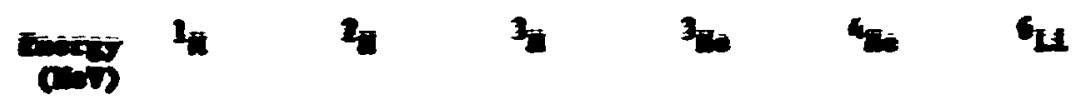

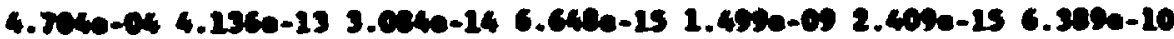
4.6310-04 4.706-13 3.5120-14 7.576e-15 1.413e-es 2.743e-15 5.900e-10 5.650-04 5.1C40-13 3.057e-14 8.314e-15 1.3510-09 3.012e-15 5.70e-10 $6.043 e-045.6700-13$ 4.235e-14 9.135e-15 $1.2020-09$ 3.310e-15 5.446e-10 $6.3670-04$ 6.0110-13 4.495e-14 9.6see-15 1.236e-0s 3.510e-15 5.206a-10 7.156e-04 6.546e-13 4.096e-14 1.056e-14 1.191e-0s 3.025e-15 5.055e-10 $0.322 e-047.444 e-13$ s.c06e-14 $1.206 e-14$ 1.123e-0s 4.378e-15 4.736e-10 $9.1770-040.452 e-13 \quad 6.332 e-14 \quad 1.366 e-14$ 1.053e-0s 4.950e-15 4.452e-10 1.05ee-03 $9.534 e-13 \quad 7.157 e-14 \quad 1.543 e-14 \quad 9.792 e-10 \quad 5.590 e-15$ 4.191e-10 $1.307 e-03 \quad 1.139 e-12$ e.566e-14 $1.047 e-14 \quad 9.015 e-10 \quad 6.691 e-15 \quad 3.033 e-10$ 1.581e-03 $1.389 e-12 \quad 1.046 e-13 \quad 2.255 e-14 \quad 8.149 e-10$ e.170e-15 $3.468 e-10$ 1.882e-03 1.664e-12 $1.254 e-13 \quad 2.704 e-14 \quad 7.504 e-10 \quad 9.796 e-15 \quad 3.167 e-10$ 2.208e-03 1.963e-12 $1.481 e-13 \quad 3.193 e-14 \quad 6.846 e-10 \quad 1.157 e-14$ 2.914e-10 2.561e-03 $2.287 e-12 \quad 1.727 e-13 \quad 3.724 e-14 \quad 6.368 e-10 \quad 1.349 e-14 \quad 2.698 e-10$ $2.940 e-03 \quad 2.635 e-12 \quad 1.992 e-13 \quad 4.295 e-14 \quad 5.954 e-10 \quad 1.556 e-14 \quad 2.512 e-10$ $3.345 e-03$ 3.007e-12 2.276e-13 4.908e-14 5.526e-10 $1.778 e-142.350 e-10$ $3.777 e-03 \quad 3.403 e-12 \quad 2.579 e-13 \quad 5.560 e-14 \quad 5.224 e-10 \quad 2.015 e-14 \quad 2.208 e-10$ 4.234e-03 3.822e-12 2.901e-13 6.254e-14 4.946e-10 2.266e-14 2.082e-10 5.763e-03 4.750e-12 3.620e-13 7.805e-14 4.411e-10 $2.828 e-14 \quad 1.869 e-10$ 7.527e-03 $6.244 e-12 \quad 4.813 e-13 \quad 1.038 e-13 \quad 3.828 e-10 \quad 3.759 e-14 \quad 1.620 e-10$ 1.025e-02 8.242e-12 $6.436 e-13 \quad 1.388 e-13 \quad 3.333 e-10 \quad 5.028 e-14 \quad 1.403 e-10$ 1.511e-02 $1.150 e-11 \quad 9.164 e-13 \quad 1.979 e-13 \quad 2.804 e-10 \quad 7.193 e-14 \quad 1.178 e-10$ 2.091e-02 $1.581 e-11 \quad 1.297 e-12 \quad 2.812 e-13 \quad 2.359 e-10 \quad 1.028 e-13 \quad 9.883 e-11$ 2.646e-02 2.017e-11 $1.700 e-12 \quad 3.699 e-13 \quad 2.057 e-10 \quad 1.387 e-13 \quad 8.616 e-11$ $3.267 e-02 \quad 2.442=-11 \quad 2.114 e-12 \quad 4.617 e-13 \quad 1.868 e-10 \quad 1.766 e-13 \quad 7.925 e-11$ 3.953e-02 2.881e-11 2.570e-12 5.637e-13 $1.695 e-102.188 e-13 \quad 7.371 e-11$ 4.704e-02 3.339e-11 3.067e-12 6.759e-13 $1.574 e-10 \quad 2.666 e-13 \quad 6.874 e-11$ 5.761e-02 3.871e-11 3.696e-12 8.196e-13 $1.437 e-10 \quad 3.289 e-13 \quad 6.444 e-11$ 7.002e-02 4.490e-11 4.532e-12 $1.009 e-12 \quad 1.292 e-10 \quad 4.109 e-13 \quad 6.084 e-11$ 8.322e-02 5.119e-11 $5.458 e-12 \quad 1.220 e-12 \quad 1.138 e-10 \quad 5.068 e-13 \quad 5.886 e-11$ 9.891e-02 5.763e-11 6.513e-12 $1.458 e-12$ 9.600e-11 $6.227 e-13 \quad 5.850 e-11$ $1.307 e-01 \quad 6.696 e-11 \quad 8.301 e-12 \quad 1.870 e-12$ 8.178e-11 8.146e-13 $6.249 e-11$ 1.819e-01 8.077e-11 $1.157 e-11 \quad 2.622 e-12 \quad 7.288 e-11 \quad 1.173 e-12 \quad 8.957 e-11$ 2.075e-01 $9.153 e-11 \quad 1.470 e-11 \quad 3.352 e-12 \quad 6.416 e-11 \quad 1.549 e-12 \quad 1.633 e-10$ 2.417e-01 9.889e-11 $1.716 e-11 \quad 3.954 e-12 \quad 6.197 e-11 \quad 1.887 e-12 \quad 2.701 e-10$ 2.710e-01 1.061e-10 $1.988 e-11 \quad 4.633 e-12 \quad 5.098 e-11 \quad 2.284 e-12 \quad 2.767 e-10$ 2.940e-01 1.116e-10 2.213e-11 5.208e-12 6.005e-11 2.657e-12 $1.902 e-10$ 3.345e-01 1.178e-10 2.487e-11 5.932e-12 5.962e-11 3.160e-12 $1.180 e-10$ 3.776e-01 $1.252 e-10 \quad 2.865 e-11 \quad 6.979 e-12 \quad 6.007 e-11 \quad 3.919 e-12 \quad 7.453 e-11$ 4.234e-01 $1.325 e-10 \quad 3.270 e-11 \quad 8.142 e-12 \quad 6.012 e-11 \quad 4.891 e-12 \quad 5.493 e-11$ $5.123 e-01 \quad 1.428 e-103.917 e-11 \quad 1.007 e-11 \quad 6.193 e-11 \quad 6.730 e-12 \quad 4.251 e-11$
\end{abstract}




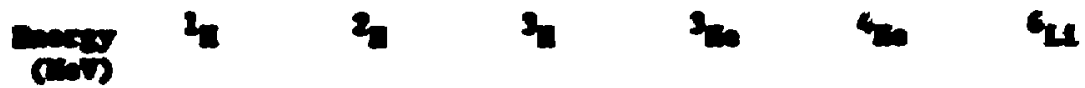

6.325e-02 $1.5730-10 \quad 4.7930-11$ 1.312a-11 $6.723 e-11 \quad 1.2260-113.4760-11$ $7.327 e-01$ 1.721e-10 5.696-11 $1.673 e-11$ 7.457e-11 $1.9040-113.076-11$

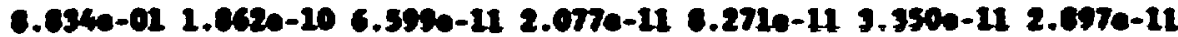
$1.0250+602.002 a-107.5640-112.5430-11$ 9.204a-11 $5.0430-112.0130-11$ $1.1760+00$ 2.14le-10 $8.4680-11$ 3.032e-11 1.018e-10 $6.1930-112.0050-11$ $1.3360+00$ 2.270-10 $9.3460-11$ 3.554e-11 $1.1210-10 \quad 6.172 e-112.8620-11$ $1.5120+002.4230-101.0260-104.130 e-11 \quad 1.225 e-10 \quad 5.567 e-11 \quad 2.967 e-11$ $1.6940+002.5430-101.110 e-104.792 e-11$ 1.331e-10 $5.1410-11$ 3.050e-11 $1.047 e+602.671 e-101.189 e-105.547 e-11$ l.421e-10 4.654e-11 $3.237 e-11$ $2.051 e+00 \quad 2.794 e-101.260 e-10 \quad 6.351 e-11 \quad 1.498 e-10 \quad 4.447 e-11 \quad 3.422 e-11$ $2.305 e+00$ 2.914e-10 $1.220 e-10 \quad 7.033 e-11 \quad 1.561 e-10 \quad 4.337 e-11 \quad 3.631 e-11$ $2.530 e+003.032 e-101.277 e-107.687 e-11$ 1.618e-10 4.185e-11 $3.822 e-11$ $2.741 e+003.142 e-101.294 e-108.269 e-11$ 1.659e-10 4.242e-11 $3.984 e-11$ $3.011 e+00 \quad 3.254 e-10 \quad 1.326 e-10 \quad 8.830 e-11 \quad 1.689 e-10 \quad 4.355 e-11 \quad 4.133 e-11$ $3.267 e+00 \quad 3.366 e-10 \quad 1.363 e-10 \quad 9.323 e-11 \quad 1.700 e-10 \quad 4.575 e-11 \quad 4.339 e-11$ $3.534 e+003.469 e-101.387 e-10 \quad 9.638 e-11 \quad 1.692 e-10 \quad 4.793 e-11 \quad 4.640 e-11$ $3.811 e+003.566 e-101.391 e-10 \quad 1.004 e-10 \quad 1.681 e-10 \quad 4.990 e-11 \quad 5.004 e-11$ 4.069e+00 3.655e-10 $1.387 e-10 \quad 1.039 e-10 \quad 1.667 e-10 \quad 5.159 e-11 \quad 5.330 e-11$ $4.396 e+003.742 e-101.369 e-10 \quad 1.063 e-10 \quad 1.668 e-10 \quad 5.328 e-115.459 e-11$ 4.704e+00 3.832e-10 $1.364 e-10 \quad 1.082 e-101.665 e-10 \quad 5.492 e-115.526 e-11$ 4.991e+00 3.906e-10 1.407e-10 1.094e-10 1.660e-10 5.626e-11 5.532e-11 $5.352 e+003.979 e-101.459 e-101.094 e-10 \quad 1.658 e-10 \quad 5.738 e-11 \quad 5.619 e-11$ $5.658 e+00 \quad 4.049 e-101.514 e-101.083 e-10 \quad 1.662 e-10 \quad 5.824 e-11 \quad 5.721 e-11$ $6.042 e+004.113 e-101.563 e-10 \quad 1.062 e-10 \quad 1.669 e-10 \quad 5.889 e-11 \quad 5.853 e-11$ $6.367 e+004.175 e-101.600 e-10 \quad 1.056 e-10 \quad 1.672 e-10 \quad 5.934 e-11 \quad 5.969 e-11$ $6.737 e+00 \quad 4.226 e-10 \quad 1.623 e-10 \quad 1.059 e-10 \quad 1.675 e-10 \quad 5.954 e-11 \quad 6.044 e-11$ $7.156 e+00 \quad 4.280 e-10 \quad 1.642 e-10 \quad 1.058 e-10 \quad 1.685 e-10 \quad 5.955 e-11 \quad 6.064 e-11$ $7.548 e+00 \quad 4.331 e-101.674 e-10 \quad 1.048 e-10 \quad 1.692 e-10 \quad 6.002 e-11 \quad 6.098 e-11$ $7.910 e+00 \quad 4.373 e-10 \quad 1.703 e-i 0 \quad 1.030 e-10 \quad 1.698 e-10 \quad 6.046 e-11 \quad 6.156 e-11$ 8.322e+00 4.410e-10 $1.727 e-10 \quad 1.013 e-10 \quad 1.705 e-10 \quad 6.087 e-11 \quad 6.239 e-11$ $8.787 e+00 \quad 4.450 e-10 \quad 1.752 e-10 \quad 1.002 e-10 \quad 1.715 e-10 \quad 6.145 e-11 \quad 6.360 e-11$ $9.177 e+00 \quad 4.486 e-10 \quad 1.772 e-10 \quad 9.874 e-11 \quad 1.731 e-10 \quad 6.185 e-11 \quad 6.463 e-11$ $9.665 e+00 \quad 4.521 e-10 \quad 1.791 e-10 \quad 9.924 e-11 \quad 1.749 e-10 \quad 6.207 e-11 \quad 6.606 e-11$ $\begin{array}{lllllll}1.012 e+01 & 4.554 e-10 & 1.807 e-10 & 9.969 e-11 & 1.759 e-10 & 6.214 e-11 & 6.771 e-11\end{array}$ $1.058 e+01 \quad 4.581 e-10 \quad 1.820 e-10 \quad 9.901 e-11 \quad 1.768 e-10 \quad 6.265 e-11 \quad 6.916 e-11$ $1.101 e+01 \quad 4.604 e-10 \quad 1.830 e-10 \quad 9.805 e-11 \quad 1.771 e-10 \quad 6.320 e-11 \quad 7.041 e-11$ $1.155 e+01 \quad 4.624 e-10 \quad 1.825 e-10 \quad 9.673 e-11 \quad 1.779 e-10 \quad 6.360 e-11 \quad 7.181 e-11$ $1.199 e+01 \quad 4.643 e-10 \quad 1.807 e-10 \quad 9.493 e-11 \quad 1.790 e-10 \quad 6.380 e-11 \quad 7.300 e-11$ $1.250 e+01 \quad 4.660 e-101.789 e-10 \quad 9.456 e-11 \quad 1.804 e-106.399 e-11 \quad 7.317 e-11$ $1.307 e+01 \quad 4.675 e-10 \quad 1.764 e-10 \quad 9.558 e-11 \quad 1.815 e-10 \quad 6.422 e-11 \quad 7.265 e-11$ $1.354 e+01 \quad 4.688 e-10 \quad 1.731 e-10 \quad 9.628 e-11 \quad 1.818 e-10 \quad 6.423 e-11 \quad 7.239 e-11$ 


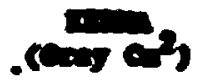

$\operatorname{man} 24$ in 4

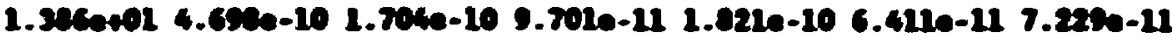

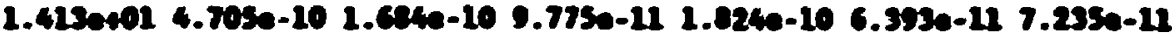

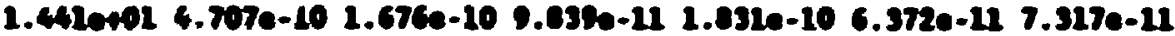

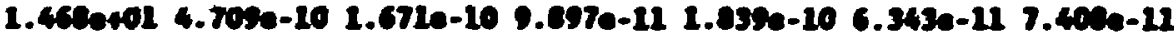

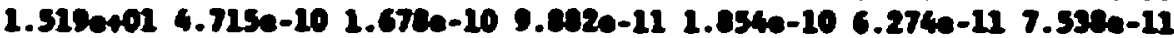

1.575er01 4.717e-10 1.650e-10 e.010e-11 $1.0710-10 \quad 6.170 e-117.702 e-11$

$1.6330+014.719 e-101.699 e-10 \quad 9.700 e-11$ 1.037e-10 $6.030 e-117.092 e-11$

1.6920401 4.717e-10 $1.703 e-10$ 9.575e-11 $1.904 e-105.8710-11$ e.189e-11

$1.752 e+01$ 4.715e-10 $1.7120-10 \quad 9.509 e-11$ 1.923e-10 $5.710 e-11 \quad 0.491 e-11$

$1.0130+01$ 4.711e-10 $1.7140-109.5540-11$ 1.946e-10 $5.496 e-11 \quad 8.779 e-11$

$1.075 e+01$ 4.704e-10 $1.710 e-10$ 9.598e-11 $1.967 e-10 \quad 5.200 e-11$ 8.945e-11

2.000e+01 4.693e-10 $1.696 e-10 \quad 9.766 e-11 \quad 1.997 e-10 \quad 4.753 e-11 \quad 9.098 e-11$ 


\section{(a)}

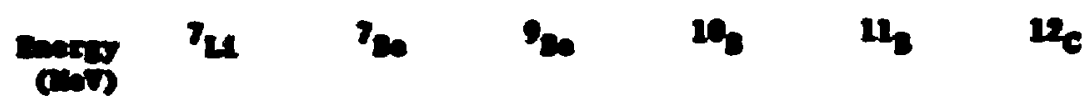

\subsection{7e-0}

5.227e-01 2.936-16 3.320e-06 6.780e-16 2.5510-07 5.425e-17 9.577e-17 2.0010-0 1.c00-16 1.626e-06 3.340e-16 1.2760-07 3.064e-17 4.320e-17

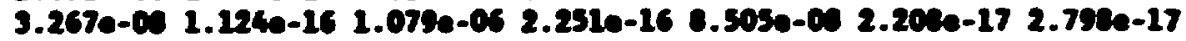
4.7060-05 9.5030-17 8.056e-07 1.090e-16 6.950-00 1.07ee-17 2.352e-17 0.322e-00 7.47\%e-17 6.942e-07 $1.4560-165.4750-001.563 e-17$ 1.e72e-17 1.307e-07 6.029e-17 5.414e-07 1.136e-16 4.257e-00 1.304e-17 1.461e-17 1.002e-07 5.150e-17 4.434e-07 9.390e-17 3.479e-08 1.150e-17 1.359e-17 2.561e-07 4.305e-17 $3.734 e-07$ e.050e-17 2.943e-0s $1.058 e-17$ 1.277e-17 3.345e-07 3.955e-17 $3.201 e-07$ 7.097e-17 2.551e-08 1.004e-17 1.181e-17 4.234e-07 3.488e-17 2.811e-07 6.405e-17 2.251e-08 9.812e-18 1.071e-17 5.123e-07 3.237e-17 2.548e-07 5.914e-17 2.025e-08 9.801e-18 9.554e-18 7.527e-07 2.948e-17 2.191e-07 5.391e-17 1.747e-08 1.014e-17 9.354e-18 1.176e-06 2.615e-17 $1.784 e-07$ 4.902e-17 $1.417 e-08$ 1.145e-17 $1.006 e-17$ 1.511e-06 2.475e-17 $1.523 e-07 \quad 4.765 e-17 \quad 1.196 e-08 \quad 1.336 e-17 \quad 1.185 e-17$ 2.091e-06 2.301e-17 $1.304 e-07 \quad 4.883 e-17 \quad 1.035 e-08 \quad 1.595 e-17 \quad 1.432 e-17$ 2.741e-06 2.272e-17 1.125e-07 5.239e-17 8.922e-09 $1.965 e-17 \quad 1.760 e-17$ 3.534e-06 2.362e-17 9.880e-08 5.811e-17 7.827e-09 2.416e-17 2.142e-17 4.704e-06 2.514e-17 8.647e-08 6.718e-17 6.834e-09 3.046e-17 2.665e-17 5.658e-06 2.784e-17 7.719e-08 7.791e-17 6.084e-09 3.736e-17 3.229e-17 6.737e-06 3.055e-17 7.064e-08 8.861e-17 $5.562 e-09 \quad 4.404 e-17 \quad 3.764 e-17$ 8.321e-06 3.410e-17 6.388e-0B $1.031 e-16 \quad 5.049 e-09 \quad 5.283 e-17 \quad 4.460 e-17$ 9.620e-06 3.803e-17 5.854e-0B $1.192 e-16 \quad 4.622 e-09 \quad 6.240 e-17 \quad 5.208 e-17$ $1.101 e-05 \quad 4.188 e-17 \quad 5.449 e-08 \quad 1.344 e-16 \quad 4.310 e-09 \quad 7.135 e-17 \quad 5.905 e-17$ 1.307e-05 4.726e-17 5.063e-0B 1.541e-16 3.990e-09 8.286e-17 6.817e-17 $\begin{array}{lllllll}1.468 e-05 & 5.302 e-17 & 4.658 e-08 & 1.753 e-16 & 3.715 e-09 & 9.513 e-17 & 7.787 e-17\end{array}$ $1.581 e-05 \quad 5.733 e-17 \quad 4.388 e-08 \quad 1.912 e-16 \quad 3.543 e-09 \quad 1.043 e-16 \quad 8.513 e-17$

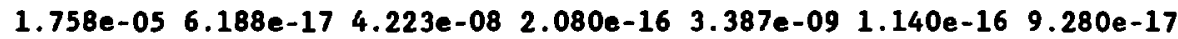

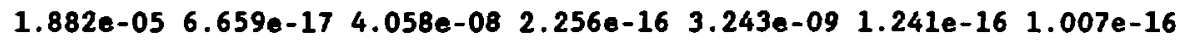
2.075e-05 7.156e-17 3.888e-08 2.441e-16 3.111e-09 $1.347 e-16 \quad 1.091 e-16$ 2.277e-05 7.794e-17 $3.727 e-08 \quad 2.672 e-16 \quad 2.966 e-09 \quad 1.480 e-16 \quad 1.196 e-16$ 2.417e-05 8.350e-17 3.599e-08 2.874e-16 2.856e-09 $1.595 e-16 \quad 1.286 e-16$ $\begin{array}{lllllll}2.561 e-05 & 8.812 e-17 & 3.492 e-08 & 3.041 e-16 & 2.773 e-09 & 1.690 e-16 & 1.361 e-16\end{array}$ 2.710e-05 9.287e-17 $3.383 e-08 \quad 3.213 e-16 \quad 2.695 e-09 \quad 1.788 e-16 \quad 1.439 e-16$ $2.862 e-05 \quad 9.775 e-17 \quad 3.270 e-08 \quad 3.391 e-16 \quad 2.621 e-09 \quad 1.890 e-16 \quad 1.518 e-16$ $2.940 e-05 \quad 1.015 e-16 \quad 3.183 e-08 \quad 3.526 e-16 \quad 2.568 e-09 \quad 1.967 e-16 \quad 1.579 e-16$

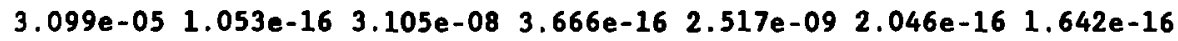
3.345e-05 $1.119 e-16 \quad 3.016 e-08 \quad 3.906 e-16 \quad 2.437 e-09 \quad 2.183 e-16 \quad 1.749 e-16$ 3.601e-05 1.201e-16 2.911e-08 4.203e-16 2.348e-09 2.351e-16 1.881e-16 $3.866 e-05 \quad 1.285 e-16 \quad 2.802 e-08 \quad 4.510 e-16 \quad 2.264 e-09 \quad 2.526 e-16 \quad 2.019 e-16$ $4.048 e-051.358 e-16 \quad 2.709 e-08 \quad 4.775 e-16 \quad 2.199 e-09 \quad 2.677 e-16 \quad 2.137 e-16$ 


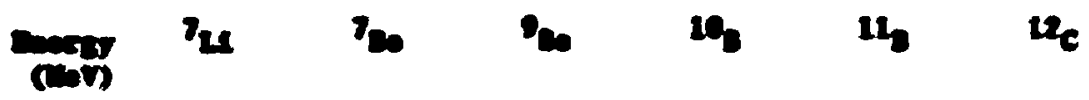

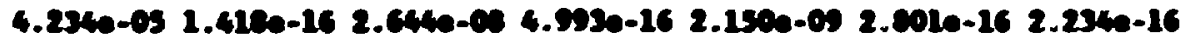

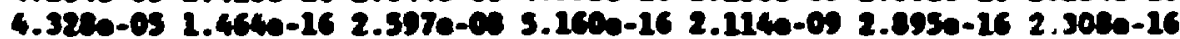

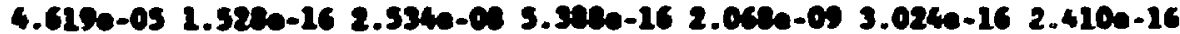
$4.7170-051.5920-162.4700-05$ 5.610-16 2.0240-09 3.1550-16 2.5120-16

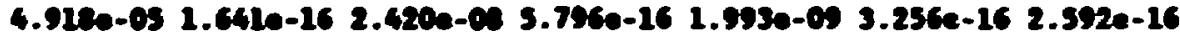
5.123e-05 1.7010-16 2.358e-05 6.036e-16 1.952e-05 3.392e-16 2.699e-16 5.332e-05 $1.777 e-16$ 2.315e-0 $6.282 e-16$ 1.913e-0s 3.532e-16 2.804e-16 5.654e-05 1.864e-16 2.262e-08 6.597e-16 1.866e-05 3.710e-16 2.948e-16 3.763e-05 1.935e-16 2.219e-08 6.053e-16 1.831e-05 3.e55e-16 3.062e-16 6.097e-05 2.002e-16 2.175e-08 7.116e-16 1.796e-0s 4.004e-16 3.179e-16 $6.325 e-05$ 2.101e-16 2.124e-05 7.450e-16 1.755e-09 4.194e-16 3.327e-16 6.557e-05 2.177e-16 2.083e-08 7.723e-16 $1.724 e-09$ 4.348e-16 3.448e-16 6.674e-05 2.235e-16 2.053e-08 7.931e-16 1.701e-09 4.466e-16 3.541e-16 7.033e-05 2.313e-16 2.011e-08 8.214e-16 1.671e-09 4.627e-16 3.666e-16 7.155e-05 2.393e-16 $1.974 e-08$ 8.501e-16 $1.642 e-09 \quad 4.789 e-16 \quad 3.793 e-16$ 7.527e-05 2.474e-16 1.947e-08 8.794e-16 1.614e-09 4.955e-16 3.924e-16 7.780e-05 2.577e-16 1.913e-08 9.166e-16 1.581e-09 5.165e-16 4.088e-16 7.908e-05 2.640e-16 1.892e-08 9.392e-16 1.562e-09 $5.293 e-16 \quad 4.189 e-16$ 8.167e-05 2.704e-16 1.868e-08 9.622e-16 1.543e-09 5.424e-16 4.291e-16 8.431e-05 2.790e-16 1.828e-08 9.933e-16 1.518e-09 $5.600 e-16 \quad 4.429 e-16$ 8.834e-05 2.900e-16 $1.777 e-08 \quad 1.033 e-15 \quad 1.489 e-09 \quad 5.824 e-16 \quad 4.604 e-16$ 9.108e-05 3.012e-16 $1.727 e-08 \quad 1.073 e-15 \quad 1.460 e-09 \quad 6.052 e-16 \quad 4.783 e-16$ 9.386e-05 3.104e-16 $1.704 e-08 \quad 1.106 e-15 \quad 1.439 e-09 \quad 6.238 e-16 \quad 4.928 e-16$ $9.668 e-05 \quad 3.196 e-16 \quad 1.685 e-08 \quad 1.139 e-15 \quad 1.417 e-09 \quad 6.426 e-16 \quad 5.076 e-16$ 9.811e-05 3.267e-16 1.671e-08 $1.165 e-15 \quad 1.402 e-09 \quad 6.570 e-16 \quad 5.188 e-16$ $1.025 e-043.362 e-16 \quad 1.652 e-08 \quad 1.199 e-15 \quad 1.381 e-09 \quad 6.764 e-16 \quad 5.340 e-16$ $1.099 e-04 \quad 3.558 e-16 \quad 1.613 e-08 \quad 1.269 e-15 \quad 1.344 e-09 \quad 7.161 e-16 \quad 5.651 e-16$ 1.176e-04 3.810e-16 $1.563 e-08 \quad 1.360 e-15 \quad 1.298 e-09 \quad 7.672 e-16 \quad 6.050 e-16$ 1.256e-04 4.070e-16 $1.511 e-08 \quad 1.453 e-15 \quad 1.255 e-09 \quad 8.200 e-16 \quad 6.463 e-16$

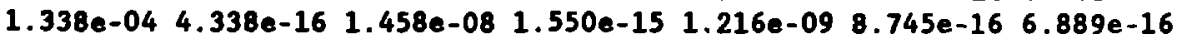
1.406e-04 4.587e-16 $1.408 e-08 \quad 1.639 e-15 \quad 1.181 e-09 \quad 9.251 e-16 \quad 7.284 e-16$ 1.511e-04 4.874e-16 $1.351 e-08 \quad 1.742 e-15 \quad 1.146 e-09 \quad 9.832 e-16 \quad 7.737 e-16$ 1.601e-04 5.197e-16 $1.301 e-08 \quad 1.858 e-15 \quad 1.110 e-09 \quad 1.049 e-15 \quad 8.250 e-16$ 1.694e-04 5.500e-16 1.265e-08 $1.967 e-15 \quad 1.078 e-09 \quad 1.110 e-15 \quad 8.731 e-16$ 1.789e-04 5.813e-16 $1.227 e-08 \quad 2.079 e-15 \quad 1.049 e-09 \quad 1.174 e-15 \quad 9.225 e-16$ 1.887e-04 6.134e-16 1.189e-08 2.194e-15 $1.021 e-09 \quad 1.239 e-15 \quad 9.732 e-16$

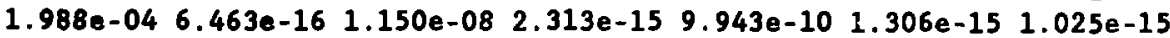
2.091e-04 6.802e-16 $1.116 e-08 \quad 2.434 e-15 \quad 9.691 e-10 \quad 1.375 e-15 \quad 1.079 e-15$ 2.741e-04 8.053e-16 $1.033 e-08 \quad 2.883 e-15 \quad 8.923 e-10 \quad 1.628 e-15 \quad 1.276 e-15$ 3.267e-04 1.001e-15 9.087e-09 3.584e-15 $7.991 e-10 \quad 2.025 e-15 \quad 1.584 e-15$ 3.811e-04 1.178e-15 8.271e-09 4.221e-15 $7.361 e-10 \quad 2.385 e-15 \quad 1.863 e-15$ 
$\cos 23$

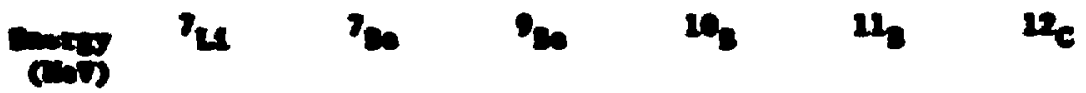

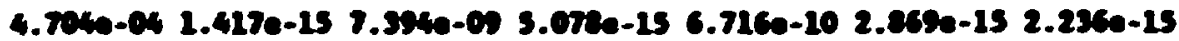
4.9210-04 1.623e-15 6.0520-0 5.7010-15 6.205e-10 3.267e-15 2.5430-15

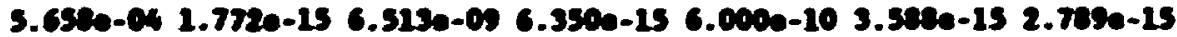
6.043e-04 1.947e-15 6.166e-05 6.976e-15 5.722e-10 3.942e-15 3.0610-15 6.367e-04 2.065e-15 5.954e-09 7.394e-15 5.956e-10 4.131e-15 3.243e-15 7.156e-04 $2.250 e-15$ s.650e-0) 8.053e-15 5.324e-10 4.556e-15 3.530e-15 $8.322 e-042.574 e-15$ 5.258e-05 9.222e-15 4.976e-10 5.215e-15 4.030e-15 $9.177 e-042.910 e-15$ 4.906e-09 1.043e-14 4.690e-10 5.895e-15 4.546e-15 1.05ee-03 3.267e-15 4.566a-05 1.17ee-14 4.406e-10 6.65ee-15 5.121e-15 $1.307 e-033.933 e-15$ 4.121e-0s $1.410 e-14$ 4.030e-10 $7.969 e-15 \quad 6.126 e-15$ 1.581e-03 4.802e-15 3.556e-03 1.722e-14 3.647e-10 $9.730 e-15 \quad 7.479 e-15$ 1.982e-03 5.758e-15 3.136e-09 2.064e-14 3.330e-10 1.167e-14 8.967e-15 2.208e-03 6.801e-15 2.784e-09 2.438e-14 3.064e-10 1.378e-14 1.059e-14 2.561e-03 7.930e-15 2.533e-09 2.843e-14 2.838e-10 1.607e-14 1.235e-14 2.940e-03 9.147e-15 $2.275 e-09 \quad 3.279 e-14 \quad 2.642 e-10 \quad 1.853 e-14 \quad 1.424 e-14$ 3.345e-03 1.045e-14 2.047e-09 3.746e-14 $2.472 e-10 \quad 2.118 e-14 \quad 1.627 e-14$ 3.777e-03 1.184e-14 1.889e-09 4.245e-14 2.323e-10 2.399e-14 1.844e-14 4.234e-03 1.332e-14 $1.729 e-094.775 e-14 \quad 2.191 e-10 \quad 2.699 e-14 \quad 2.074 e-14$ 5.763e-03 1.662e-14 1.477e-09 5.959e-14 $1.967 e-10 \quad 3.368 e-14 \quad 2.588 e-14$ 7.527e-03 2.209e-14 $1.212 e-09 \quad 7.921 e-14 \quad 1.706 e-10 \quad 4.477 e-14 \quad 3.493 e-14$ 1.025e-02 2.954e-14 9.720e-10 $1.059 e-13 \quad 1.478 e-10 \quad 5.987 e-14 \quad 4.737 e-14$ $1.511 e-02 \quad 4.202 e-14 \quad 7.642 e-10 \quad 1.500 e-13 \quad 1.242 e-10 \quad 8.541 e-14 \quad 6.634 e-14$ 2.091e-02 5.938e-14 5.782e-10 2.104e-13 $1.043 e-10 \quad 1.213 e-13 \quad 9.425 e-14$ $2.646 e-027.765 e-14 \quad 4.685 e-10 \quad 2.729 e-13 \quad 9.109 e-11 \quad 1.586 e-13 \quad 1.240 e-13$ $3.267 e-02$ 9.635e-14 $3.870 e-10 \quad 3.381 e-13 \quad 8.174 e-11 \quad 1.960 e-13 \quad 1.631 e-13$ $3.953 e-02 \quad 1.169 e-13 \quad 3.267 e-10 \quad 4.106 e-13 \quad 7.420 e-11 \quad 2.367 e-13 \quad 1.912 e-13$ 4.704e-02 1.391e-13 2.767e-10 4.893e-13 6.800e-11 $2.803 e-13 \quad 2.241 e-13$ 5.761e-02 1.662e-13 2.326e-10 $5.860 e-13 \quad 6.216 e-11 \quad 3.340 e-13 \quad 2.709 e-13$ 7.002e-02 $1.988 e-13 \quad 1.918 e-10 \quad 7.034 e-13 \quad 5.664 e-11 \quad 4.013 e-13 \quad 3.303 e-13$ 8.322e-02 2.341e-13 $1.622 e-10 \quad 8.293 e-13 \quad 5.204 e-11 \quad 4.738 e-13 \quad 3.966 e-13$ 9.891e-02 2.718e-13 $1.370 e-10 \quad 9.652 e-13 \quad 4.814 e-11 \quad 5.525 e-13 \quad 4.713 e-13$ 1.307e-01 3.149e-13 $1.144 \mathrm{e}-10 \quad 1.177 \mathrm{e}-12 \quad 4.354 \mathrm{e}-11 \quad 6.740 \mathrm{e}-13 \quad 5.860 \mathrm{e}-13$ $1.819 e-01 \quad 4.100 e-13 \quad 8.794 e-11 \quad 1.513 e-12 \quad 3.835 e-11 \quad 8.690 e-13 \quad 7.644 e-13$ $\begin{array}{lllllll}2.075 e-01 & 6.147 e-13 & 7.433 e-11 & 1.786 e-12 & 3.517 e-11 & 1.042 e-12 & 9.107 e-13\end{array}$ $2.417 \mathrm{e}-01 \quad 1.855 \mathrm{e}-12 \quad 7.080 \mathrm{e}-11 \quad 1.966 \mathrm{e}-12 \quad 3.258 \mathrm{e}-11 \quad 1.173 \mathrm{e}-12 \quad 1.024 \mathrm{e}-12$ $2.710 e-01 \quad 6.773 e-12 \quad 7.358 e-11 \quad 2.118 e-12 \quad 3.150 e-11 \quad 1.306 e-12 \quad 1.140 e-12$ 2.940e-01 5.011e-12 $7.886 e-11 \quad 2.251 e-12 \quad 3.035 e-11 \quad 1.409 e-12 \quad 1.232 e-12$ $3.345 e-01 \quad 2.830 e-12 \quad 8.413 e-11 \quad 2.416 e-12 \quad 2.828 e-11 \quad 1.527 e-12 \quad 1.337 e-12$ 3.776e-01 $1.964 e-12 \quad 8.922 e-11 \quad 2.631 e-12 \quad 2.583 e-11 \quad 1.689 e-12 \quad 1.468 e-12$ 4.234e-01 $1.863 e-12 \quad 8.758 e-11 \quad 2.833 e-12 \quad 2.470 e-11 \quad 2.316 e-12 \quad 1.595 e-12$

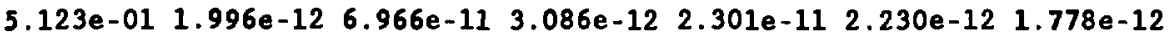




\title{
$\cos ^{2}$
}

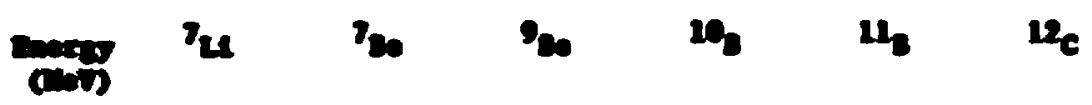

\begin{abstract}
6.325e-01 2.319e-12 4.773e-12 4.031e-12 $1.970-121.916-122.031 e-12$ 7.527e-02 2.753e-12 3.6940-11 4.7120-12 $1.5000-11$ 1.906-12 $2.356 e-12$

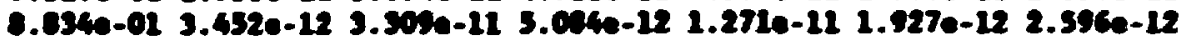
1.025er00 4.310e-22 3.001e-11 5.003e-12 $1.0000-11$ 1.022e-12 $2.760 e-12$ 1.176en00 5.297e-12 2.975e-12 5.407e-12 1.051e-11 $2.967 e-12$ 2.979e-12 $1.3340400 \quad 6.323 e-12$ 2.096e-11 $5.444 e-12$ 1.127e-12 $6.119 e-12 \quad 3.222 e-12$ $1.51104007 .1210-12$ 2.705e-11 $5.246 e-12 \quad 1.2240-11 \quad 5.040 e-12 \quad 3.425 e-12$ $1.6940+007.940 e-12$ 2.016e-11 $5.207 e-12$ 1.640s-11 4.356e-12 3.564e-12 $1.887 e+00 \quad 8.709 e-12 \quad 2.979 e-12 \quad 5.451 e-12 \quad 2.145 e-12 \quad 4.727 e-12 \quad 3.766 e-12$ 2.091e+00 9.712e-12 3.144e-11 6.328e-12 2.241e-11 4.655e-12 4.521e-12 $2.305 e+001.105 e-11 \quad 3.414 e-11 \quad 8.253 e-12 \quad 1.920 e-11 \quad 5.145 e-12 \quad 4.105 e-12$ $2.530 e+00 \quad 1.259 e-11 \quad 3.714 e-11 \quad 1.182 e-11 \quad 1.003 e-11 \quad 7.271 e-12 \quad 4.402 e-12$ 2.741e+00 1.400e-11 4.004e-11 1.501e-11 $1.887 e-11 \quad 7.093 e-12 \quad 5.320 e-12$ $3.011 e+001.549 e-11 \quad 4.318 e-11 \quad 1.422 e-11 \quad 1.906 e-11 \quad 6.400 e-12 \quad 7.951 e-12$ $3.267 e+001.721 e-11 \quad 4.814 e-11 \quad 1.406 e-11 \quad 1.820 e-11 \quad 5.968 e-12 \quad 6.713 e-12$ $3.534 e+001.908 e-11 \quad 5.461 e-11 \quad 1.440 e-11 \quad 1.806 e-11$ 5.491e-12 $1.021 e-11$ 3.811e+00 2.106e-11 6.142e-11 1.488e-11 1.849e-11 5.132e-12 1.051e-11 $4.069 e+002.347 e-11 \quad 6.838 e-11 \quad 1.533 e-11 \quad 2.065 e-11 \quad 4.869 e-12 \quad 8.958 e-12$ $4.396 e+00$ 2.687e-11 $7.923 e-11 \quad 1.612 e-11$ 2.310e-11 $5.017 e-12$ 9.357e-12 4.704e+00 2.936e-11 $9.235 e-11 \quad 1.686 e-11 \quad 2.288 e-11 \quad 5.835 e-12 \quad 8.120 e-12$ $4.991 e+00 \quad 3.082 e-11 \quad 1.049 e-10 \quad 1.699 e-11 \quad 2.225 e-11 \quad 6.450 e-12 \quad 7.122 e-12$ 5.352e+00 3.220e-11 $1.206 e-10 \quad 1.737 e-11 \quad 2.288 e-11 \quad 6.578 e-12 \quad 6.925 e-12$ 5.658e+00 3.419e-11 $1.386 e-10 \quad 1.792 e-11 \quad 2.493 e-11 \quad 6.395 e-12 \quad 7.222 e-12$ $\begin{array}{lllllll}6.042 e+00 & 3.758 e-11 & 1.574 e-10 & 1.843 e-11 & 2.722 e-11 & 6.347 e-12 & 6.338 e-12\end{array}$ $6.367 e+00 \quad 3.963 e-11 \quad 1.702 e-10 \quad 1.896 e-11 \quad 2.891 e-11 \quad 6.576 e-12 \quad 8.078 e-12$ 6.737e+00 4.035e-11 $1.779 e-10 \quad 1.949 e-11 \quad 3.021 e-11 \quad 6.317 e-12 \quad 5.255 e-12$ $7.156 e+00 \quad 4.103 e-11 \quad 1.866 e-10 \quad 2.028 e-11 \quad 3.149 e-11 \quad 6.274 e-12 \quad 4.567 e-12$

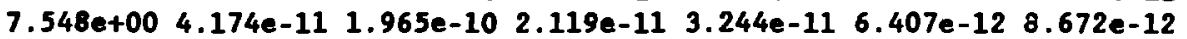
7.910e+00 4.239e-11 2.061e-10 2.199e-11 3.322e-11 6.691e-12 $1.270 e-11$ $8.322 e+00 \quad 4.304 e-11 \quad 2.147 e-10 \quad 2.277 e-11 \quad 3.406 e-11 \quad 7.236 e-12 \quad 1.229 e-11$ 8.787e+00 4.401e-11 2.219e-10 2.372e-11 3.520e-11 8.460e-12 9.010e-12 $9.177 e+00 \quad 4.486 e-11 \quad 2.288 e-10 \quad 2.479 e-11 \quad 3.632 e-11 \quad 1.025 e-11 \quad 1.003 e-11$ $9.665 e+00 \quad 4.580 e-11 \quad 2.360 e-10 \quad 2.577 e-11 \quad 3.748 e-11 \quad 1.212 e-11 \quad 1.136 e-11$ $1.012 e+01 \quad 4.671 e-11 \quad 2.436 e-10 \quad 2.679 e-11 \quad 3.872 e-11 \quad 1.418 e-11 \quad 1.147 e-11$ $1.058 e+01 \quad 4.745 e-112.498 e-10 \quad 2.768 e-11 \quad 4.012 e-11 \quad 1.595 e-11 \quad 1.166 e-11$ $1.101 e+01 \quad 4.810 e-11 \quad 2.554 e-10 \quad 2.844 e-11 \quad 4.156 e-11 \quad 1.772 e-11 \quad 1.392 e-11$ $1.155 e+01 \quad 4.881 e-11 \quad 2.614 e-10 \quad 2.892 e-11 \quad 4.313 e-11 \quad 1.958 e-11 \quad 1.546 e-11$ $1.199 e+01 \quad 4.956 e-11 \quad 2.674 e-10 \quad 2.919 e-11 \quad 4.477 e-11 \quad 2.348 e-11 \quad 1.627 e-11$ $1.250 e+01 \quad 4.945 e-11 \quad 2.733 e-10 \quad 2.972 e-11 \quad 4.630 e-11 \quad 2.300 e-11 \quad 1.787 e-11$ $\begin{array}{lllllll}1.307 e+01 & 4.846 e-11 & 2.800 e-10 & 3.059 e-11 & 4.792 e-11 & 2.435 e-11 & 1.856 e-11\end{array}$ $1.354 e+01$ 4.765e-11 2.864e-10 3.145e-11 4.948e-11 2.574e-11 $1.936 e-11$
\end{abstract}




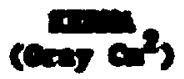

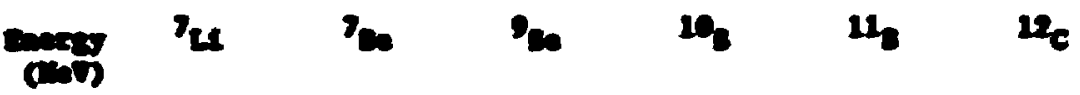

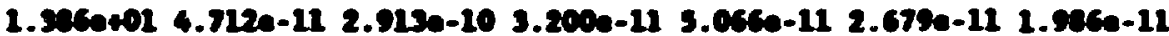
1.4230*01 4.677e-11 $2.9450-103.242 e-12$ s.154e-11 $2.7540-12$ 2.023e-11 1.4010+01 4.6ese-11 2.502e-10 3.252e-11 5.236e-11 $2.0020-112.056 e-11$ 1.464at01 4.710e-11 3.016e-10 3.346e-11 5.317e-11 $2.047 e-112.0550-11$ 1.525e+01 4.734e-11 3.064e-10 3.453e-21 5.405e-11 $2.926 e-112.2030-11$ $1.575 e+01$ 4.740e-11 $3.226 e-103.6340-11$ 5.461e-11 3.036e-11 $2.3360-11$ $1.63304014 .787 e-113.200 e-103.033 e-11$ 5.560e-11 $3.151 e-112.447 e-11$ $1.692 e+015.047 e-11$ 3.261e-10 4.021e-11 $5.688 e-11 \quad 3.266 e-112.404 e-11$ $1.752 e+015.324 e-11 \quad 3.365 e-10 \quad 4.192 e-11$ 5.622e-11 $3.381 e-112.495 e-11$ $1.013 e+01$ 5.585e-11 3.451e-10 4.302e-21 5.959e-11 3.497e-11 2.581e-11 $1.875 e+01 \quad 5.730 e-11 \quad 3.540 e-104.384 e-11 \quad 6.073 e-11 \quad 3.611 e-11 \quad 2.725 e-11$ 2.000e+01 5.866e-11 3.675e-10 4.470e-11 6.221e-11 3.776e-11 2.902e-11 


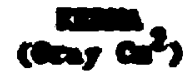

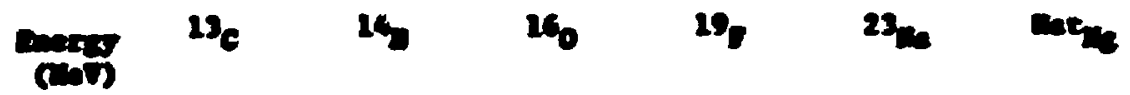

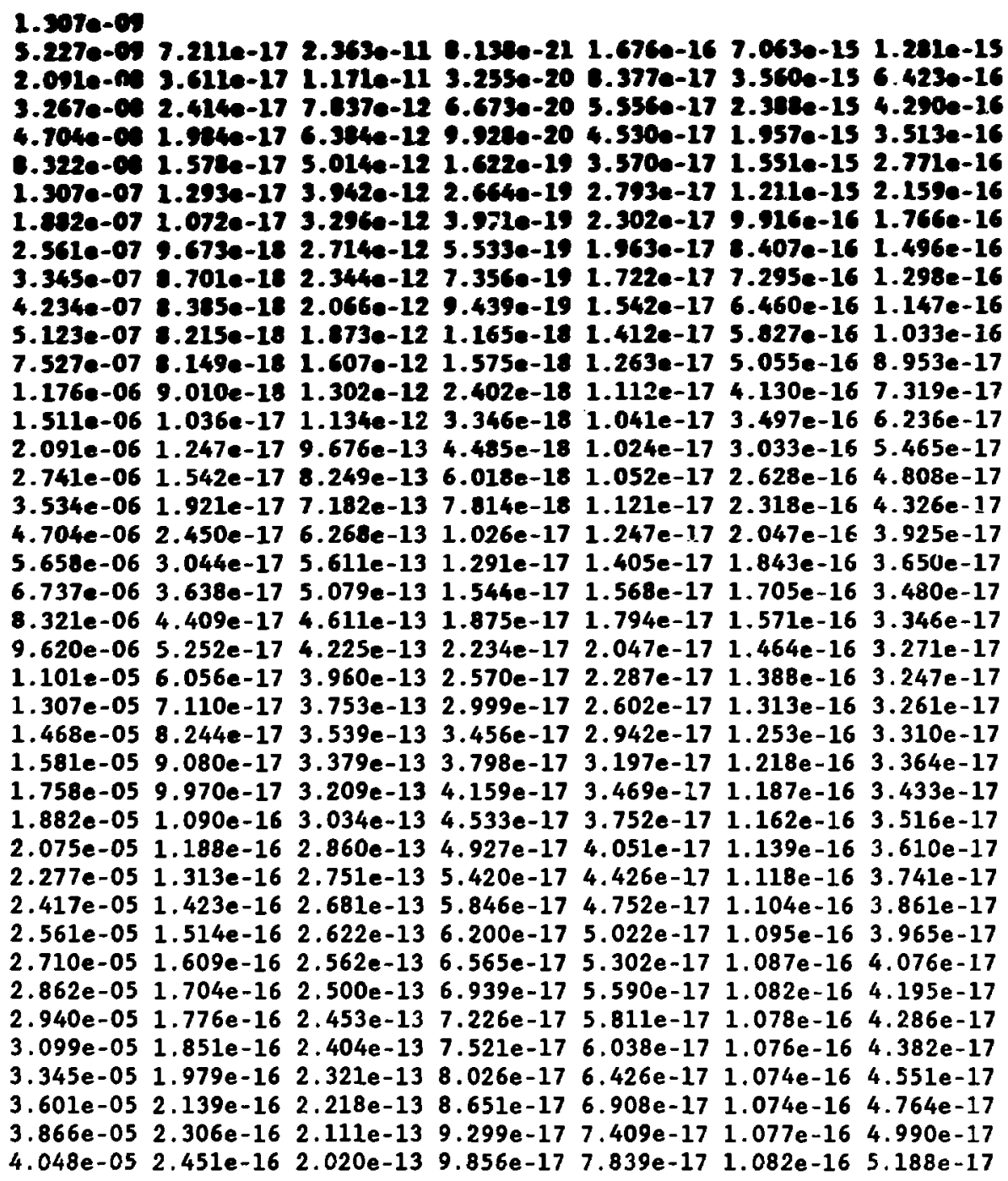




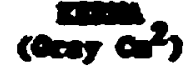

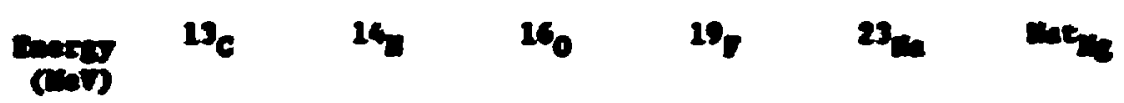

4.234e-05 $2.5700-16 \quad 1.975 e-13 \quad 1.0310-16 \quad 8.193 e-17 \quad 1.037 a-16 \quad 5.3530-17$ 4.3200-05 2.6520-16 $1.950 e-131.066 e-16$ e.464e-17 $1.0910-165.4790-17$ 4.619e-05 2.707e-16 $1.915 e-13 \quad 1.114 e-16$ e.035e-17 $1.0940-16$ 5.654e-17 4.717e-05 2.915e-16 $1.079 e-13 \quad 1.163 e-16 \quad 9.210 e-17 \quad 1.106 e-16$ 5.833e-17 4.916e-05 3.014e-16 $1.052 e-13 \quad 1.200 e-16 \quad 9.493 e-17 \quad 1.113 e-16 \quad 5.971 e-17$ 5.123e-05 3.14e-16 $1.0150-13 \quad 1.250 e-16 \quad 9.8900-17 \quad 1.122 e-16 \quad 6.160 e-17$ 5.332e-05 3.246e-16 $1.777 e-13 \quad 1.302 e-16 \quad 1.028 e-16 \quad 1.133 e-16 \quad 6.354 e-17$ 5.654e-05 3.464e-16 $1.729 e-13 \quad 1.364 e-16 \quad 1.090 e-16 \quad 1.147 e-16 \quad 6.604 e-17$ 5.763e-05 3.609e-16 $1.629 e-13 \quad 1.422 e-16 \quad 1.122 e-16 \quad 1.159 e-16 \quad 6.809 e-17$ $6.097 e-05 \quad 3.759 e-16 \quad 1.650 e-13 \quad 1.477 e-16 \quad 1.165 e-16 \quad 1.173 e-16 \quad 7.021 e-17$ 6.325e-05 3.950e-16 $1.613 e-13 \quad 1.547 e-16 \quad 1.219 e-16 \quad 1.191 e-16 \quad 7.289 e-17$ 6.557e-05 4.107e-16 1.588e-13 $1.604 e-16 \quad 1.264 e-16 \quad 1.206 e-16 \quad 7.511 e-17$ 6.674e-05 4.228e-16 1.569e-13 $1.648 e-16 \quad 1.297 e-16 \quad 1.218 e-16 \quad 7.681 e-17$ 7.033e-05 4.391e-16 $1.543 e-13 \quad 1.707 e-16 \quad 1.344 e-16 \quad 1.235 e-16 \quad 7.912 e-17$ $7.155 e-05 \quad 4.552 e-16 \quad 1.517 e-13 \quad 1.767 e-16 \quad 1.390 e-16 \quad 1.252 e-16 \quad 8.145 e-17$ $7.527 e-05 \quad 4.716 e-16 \quad 1.490 e-13 \quad 1.829 e-16 \quad 1.438 e-16 \quad 1.270 e-16 \quad 8.386 e-17$ $7.780 e-05 \quad 4.924 e-16 \quad 1.456 e-13 \quad 1.906 e-16 \quad 1.498 e-16 \quad 1.293 e-16 \quad 8.692 e-17$ 7.908e-05 5.051e-16 $1.435 e-13 \quad 1.954 e-16 \quad 1.535 e-16 \quad 1.308 e-16 \quad 8.878 e-17$ 8.167e-05 5.181e-16 $1.416 e-13 \quad 2.002 e-16 \quad 1.573 e-16 \quad 1.323 e-16 \quad 9.069 e-17$ 8.431e-05 5.356e-16 $1.396 e-13 \quad 2.067 e-16 \quad 1.623 e-16 \quad 1.344 e-16 \quad 9.328 e-17$ 8.834e-05 5.581e-16 $1.370 e-13 \quad 2.150 e-16 \quad 1.688 e-16 \quad 1.372 e-16 \quad 9.658 e-17$ 9.108e-05 5.809e-16 1.345e-13 2.235e-16 $1.753 e-16 \quad 1.400 e-16 \quad 9.994 e-17$ $9.386 e-05 \quad 5.996 e-16 \quad 1.325 e-13 \quad 2.303 e-16 \quad 1.807 e-16 \quad 1.423 e-16 \quad 1.027 e-16$ $9.668 e-05 \quad 6.187 e-16 \quad 1.304 e-13 \quad 2.373 e-16 \quad 1.861 e-16 \quad 1.446 e-16 \quad 1.055 e-16$ 9.811e-05 6.331e-16 $1.288 e-13 \quad 2.426 e-16 \quad 1.902 e-16 \quad 1.464 e-16 \quad 1.076 e-16$ $1.025 e-04 \quad 6.528 e-16 \quad 1.269 e-13 \quad 2.498 e-16 \quad 1.958 e-16 \quad 1.489 e-16 \quad 1.104 e-16$

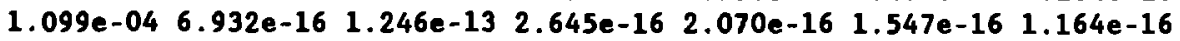
$1.176 e-04 \quad 7.456 e-16 \quad 1.219 e-13 \quad 2.833 e-16 \quad 2.213 e-16 \quad 1.623 e-16 \quad 1.241 e-16$ $1.256 e-048.001 e-16 \quad 1.191 e-13 \quad 3.029 e-16 \quad 2.360 e-16 \quad 1.702 e-16 \quad 1.320 e-16$ $1.338 e-048.568 e-16 \quad 1.162 e-13 \quad 3.231 e-16 \quad 2.512 e-16 \quad 1.783 e-16 \quad 1.402 e-16$ $1.406 e-049.097 e-16 \quad 1.135 e-13 \quad 3.417 e-16 \quad 2.653 e-16 \quad 1.859 e-16 \quad 1.478 e-16$ $1.511 e-04 \quad 9.711 e-16 \quad 1.104 e-13 \quad 3.632 e-16 \quad 2.813 e-16 \quad 1.946 e-16 \quad 1.565 e-16$ $\begin{array}{lllllll}1.601 e-04 & 1.041 e-15 & 1.069 e-13 & 3.875 e-16 & 2.994 e-16 & 2.044 e-16 & 1.663 e-16\end{array}$

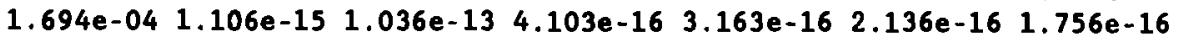

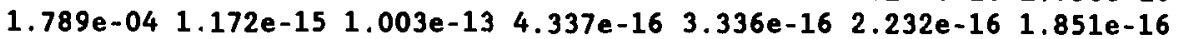

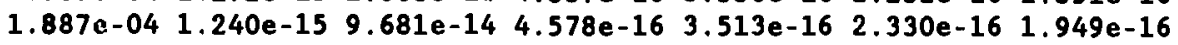
$1.988 e-04 \quad 1.310 e-15 \quad 9.324 e-14 \quad 4.825 e-16 \quad 3.693 e-16 \quad 2.430 e-16 \quad 2.049 e-16$ $2.091 e-04 \quad 1.382 e-15 \quad 9.053 e-14 \quad 5.079 e-16 \quad 3.878 e-16 \quad 2.534 e-16 \quad 2.153 e-16$ $2.741 e-04 \quad 1.653 e-15 \quad 8.586 e-14 \quad 6.018 e-16 \quad 4.552 e-16 \quad 2.918 e-16 \quad 2.539 e-16$ 3.267e-04 2.083e-15 $7.858 e-14 \quad 7.482 e-16 \quad 5.582 e-16 \quad 3.525 e-16 \quad 3.142 e-16$ 3.811e-04 2.473e-15 7.196e-14 8.814e-16 6.492e-16 4.113e-16 3.690e-16 


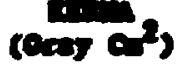
$\sin$
14.
160
19.

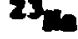

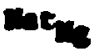

$4.7040-042.9060-15$ 6.509e-14 $1.0510-157.675 e-16$ 4.920e-16 4.426e-16 4.991e-04 3.434e-15 6.196e-14 1.207e-15 e.616e-16 5.391e-16 5.033e-16

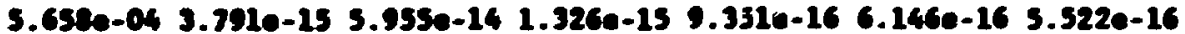
6.043e-04 4.10ee-15 5.696e-14 1.457e-15 1.014e-15 6.766e-16 6.060e-16 $6.367 e-04$ 4.456e-15 $5.557 e-14 \quad 1.545 e-15$ l.066e-15 $7.222 e-16 \quad 6.423 e-16$ $7.156 e-04$ 4.006e-15 5.406e-14 1.624e-15 $1.146 e-15 \quad 7.996 e-16 \quad 6.993 e-16$ $0.322 e-045.649 e-15$ 5.147e-14 $1.929 e-15$ 1.305e-15 $9.425 e-167.993 e-16$ $9.177 e-04 \quad 6.430 e-15$ 4.956e-14 2.179e-15 $1.476 e-15 \quad 1.097 e-15 \quad 9.030 e-16$ $1.058 e-037.298 e-15 \quad 4.796 e-14 \quad 2.461 e-15 \quad 1.667 e-15 \quad 1.281 e-15 \quad 1.020 e-15$ $1.307 e-03 \quad 8.809 a-15 \quad 4.682 e-14 \quad 2.946 e-15 \quad 1.995 e-15 \quad 1.729 e-15 \quad 1.232 e-15$ 1.581e-03 1.087e-14 4.564e-14 3.597e-15 2.436e-15 2.598e-15 1.524e-15 $1.882 e-031.320 e-14 \quad 4.429 e-14 \quad 4.313 e-15 \quad 2.921 e-15 \quad 4.905 e-151.852 e-15$ 2.208e-03 1.575e-14 $4.328 e-14 \quad 5.094 e-15 \quad 3.451 e-15 \quad 1.186 e-14 \quad 2.212 e-15$ $2.561 e-031.850 e-14 \quad 4.405 e-145.940 e-15$ 4.024e-15 4.653e-14 2.582e-15 2.940e-03 2.149e-14 4.500e-14 6.852e-15 4.642e-15 2.542e-13 2.977e-15 3.345e-03 2.475e-14 4.598e-14 7.828e-15 5.305e-15 $1.708 e-13$ 3.401e-15 3.777e-03 2.827e-14 4.700e-14 8.870e-15 6.011e-15 5.562e-14 3.851e-15 $4.234 e-03 \quad 3.199 e-14 \quad 4.818 e-14 \quad 9.976 e-15 \quad 6.762 e-15 \quad 3.093 e-14 \quad 4.328 e-15$ 5.763e-03 4.026e-14 5.257e-14 1.245e-14 B.442e-15 $1.965 e-14 \quad 5.386 e-15$ 7.527e-03 5.427e-14 $6.023 e-14 \quad 1.655 e-14 \quad 1.123 e-14 \quad 1.760 e-14 \quad 7.126 e-15$ $1.025 e-02 \quad 7.393 e-14 \quad 7.156 e-14 \quad 2.213 e-14 \quad 1.503 e-14 \quad 1.691 e-14 \quad 9.594 e-15$ 1.511e-02 1.077e-13 $9.220 e-14 \quad 3.157 e-14 \quad 2.130 e-14 \quad 2.000 e-14 \quad 1.406 e-14$ 2.091e-02 1.555e-13 1.179e-13 4.485e-14 3.000e-14 2.558e-14 2.893e-14 2.646e-02 2.081e-13 $1.432 e-13 \quad 5.899 e-14 \quad 4.353 e-14 \quad 3.254 e-14 \quad 3.624 e-14$ $3.267 e-022.635 e-13 \quad 1.675 e-13 \quad 7.364 e-14 \quad 1.529 e-13 \quad 3.946 e-14 \quad 2.795 e-14$ $3.953 e-02 \quad 3.248 e-13 \quad 1.933 e-13 \quad 8.992 e-14 \quad 5.835 e-14 \quad 5.372 e-14 \quad 3.775 e-14$ 4.704e-02 $3.933 e-13 \quad 2.202 e-13 \quad 1.078 e-13 \quad 9.026 e-14 \quad 5.644 e-14 \quad 5.021 e-14$ $5.761 e-02 \quad 4.813 e-13 \quad 2.534 e-13 \quad 1.303 e-13 \quad 3.144 e-13 \quad 1.598 e-13 \quad 5.419 e-14$ 7.002e-02 5.919e-13 2.910e-13 1.590e-13 9.879e-14 8.270e-14 8.410e-14 8.322e-02 $7.160 e-13 \quad 3.269 e-13 \quad 1.908 e-13 \quad 1.394 e-13 \quad 9.432 e-14 \quad 3.972 e-13$ 9.891e-02 8.581e-13 $3.615 e-13 \quad 2.268 e-13 \quad 5.669 e-13 \quad 1.036 e-13 \quad 6.572 e-13$

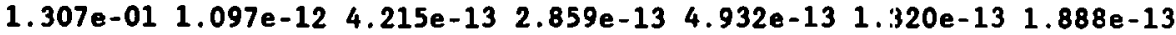

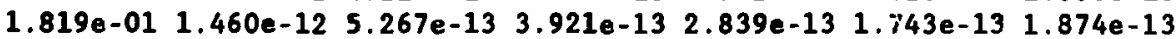
$2.075 e-01 \quad 1.559 e-12 \quad 6.244 e-13 \quad 5.076 e-13 \quad 3.213 e-13 \quad 2.339 e-13 \quad 2.449 e-13$ 2.417e-01 $1.614 \mathrm{e}-12 \quad 6.886 \mathrm{e}-13 \quad 6.070 \mathrm{e}-13 \quad 4.704 \mathrm{e}-13 \quad 3.443 \mathrm{e}-13 \quad 4.006 \mathrm{e}-13$ $2.710 e-01 \quad 1.661 e-12 \quad 7.468 e-13 \quad 7.196 e-13 \quad 7.402 e-13 \quad 3.874 e-13 \quad 6.864 e-13$ 2.940e-01 1.703e-12 7.875e-13 $8.164 e-13 \quad 1.145 e-12 \quad 2.621 e-13 \quad 6.751 e-13$ 3.345e-01 1.750e-12 8.375e-13 9.403e-13 $1.044 e-12 \quad 3.386 e-13 \quad 5.839 e-13$ $\begin{array}{lllllll}3.776 e-01 & 1.800 e-12 & 9.080 e-13 & 1.252 e-12 & 1.134 e-12 & 3.691 e-13 & 4.330 e-13\end{array}$ $4.234 e-01 \quad 1.860 e-12 \quad 9.868 e-13 \quad 2.081 e-12 \quad 1.299 e-12 \quad 5.364 e-13 \quad 5.733 e-13$ $5.123 e-01 \quad 1.932 e-12 \quad 1.420 e-12 \quad 2.284 e-12 \quad 1.047 e-12 \quad 4.438 e-13 \quad 8.343 e-13$ 


\section{$(\cos \theta)$}

$\sin$

$13 c$

14

160

19.

23.

24

6.325 3-01 2.021e-12 $1.163 e-129.523 e-13 \quad 1.046 e-12 \div .789 e-13 \quad 5.652 e-13$ 7.527e-01 2.112e-12 $2.047 e-12 \quad 1.202 e-12 \quad 9.064 e-13 \quad 1.119 e-12 \quad 6.043 e-13$ 6.834-01 2.196e-12 $1.309 e-12 \quad 1.534 e-12 \quad 1.239 e-12 \quad 9.075 e-13 \quad 6.083 e-13$ 1.025e+00 2.293e-12 $1.244 e-123.696 e-12 \quad 1.271 e-12$ $1.176 e+002.400 e-12 \quad 1.696 e-12 \quad 3.192 e-12 \quad 1.466 e-12 \quad 9.562 e-13 \quad 6.301 e-13$

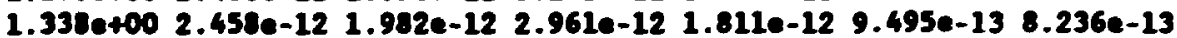
$1.511 e+00 \quad 2.466 e-12 \quad 4.498 e-12 \quad 1.873 e-12 \quad 1.638 e-12 \quad 8.302 e-13 \quad 7.645 e-13$ $1.694 e+002.465 e-12 \quad 3.068 e-12 \quad 2.206 e-12 \quad 2.055 e-12 \quad 9.216 e-13 \quad 7.311 e-13$ $1.887 e+00 \quad 3.244 e-12 \quad 4.233 e-12 \quad 2.201 e-12 \quad 1.917 e-12 \quad 1.060 e-12 \quad 1.084 e-12$ 2.091e+00 3.181e-12 3.420e-12 2.340 e-12 $2.288 e-12 \quad 1.215 e-12 \quad 1.108 e-12$ 2.305e+00 3.202e-12 4.526e-12 $1.634 e-12 \quad 2.165 e-12 \quad 1.432 e-12 \quad 1.173 e-12$ $2.530 e+00 \quad 4.404 e-12 \quad 4.622 e-12 \quad 9.695 e-13 \quad 2.528 e-12$ i.500e-12 $1.078 e-12$ $\begin{array}{lllllll}2.741 e+00 & 4.077 e-12 & 6.049 e-12 & 1.878 e-12 & 2.889 e-12 & 1.421 e-12 & 1.518 e-12\end{array}$ $\begin{array}{lllllll}3.011 e+00 & 4.474 e-12 & 7.956 e-12 & 2.112 e-12 & 2.628 e-12 & 1.421 e-12 & 1.344 e-12\end{array}$

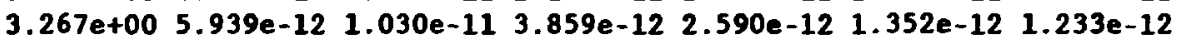
$3.534 e+00 \quad 9.349 e-12 \quad 1.203 e-11 \quad 6.114 e-12 \quad 2.561 e-12 \quad 1.598 e-12 \quad 1.437 e-12$ $3.811 e+00 \quad 8.607 e-12 \quad 1.322 e-11 \quad 5.693 e-12 \quad 2.706 e-12 \quad 1.766 e-12 \quad 1.490 e-12$ $4.069 e+00 \quad 6.703 e-12 \quad 1.533 e-11 \quad 4.772 e-12 \quad 2.715 e-12 \quad 1.620 e-12 \quad 1.341 e-12$

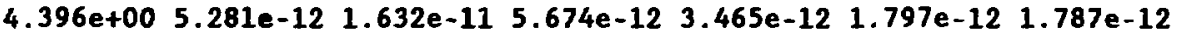
$4.704 e+00 \quad 4.522 e-12 \quad 1.444 e-11 \quad 4.745 e-12 \quad 4.371 e-12 \quad 1.767 e-12 \quad 1.804 e-12$ $4.991 e+00 \quad 6.880 e-12 \quad 1.081 e-11 \quad 4.447 e-12 \quad 4.425 e-12 \quad 1.833 e-12 \quad 1.873 e-12$ $5.352 e+00 \quad 5.180 e-12 \quad 1.223 e-11 \quad 5.475 e-12 \quad 5.136 e-12 \quad 1.960 e-i 2 \quad 1.817 e-12$

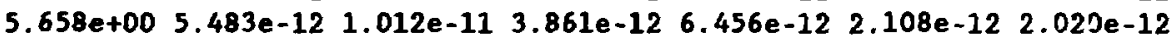

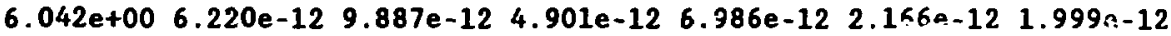
$\begin{array}{llllllll}6.367 e+00 & 6.882 e-12 & 1.240 e-11 & 3.970 e-12 & 6.524 e-12 & 2.4 \cdots & -12 & 1.929 e-12\end{array}$ $\begin{array}{lllllll}6.737 e+00 & 7.108 e-12 & 9.3^{n} 2 e-12 & 5.678 e-12 & 6.214 e-12 & 2.450 e-12 & 2.427 e-12\end{array}$ $7.156 e+00 \quad 6.702 e-12 \quad 9.457 e-12 \quad 5.584 e-12 \quad 6.275 e-12 \quad 2.533 e-12 \quad 2.747 e-12$ $\begin{array}{lllllll}7.548 e+00 & 6.181 e-12 & 1.280 e-11 & 7.150 e-12 & 6.681 e-12 & 2.867 e-12 & 3.030 e-12\end{array}$

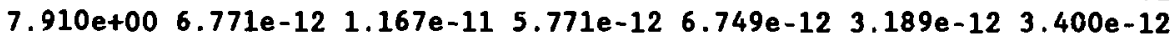

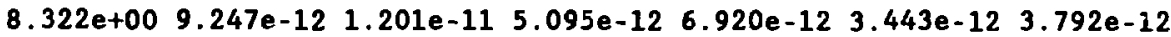
$8.787 e+00 \quad 8.690 e-12 \quad 1.080 e-11 \quad 5.729 e-12 \quad 7.318 e-12 \quad 3.771 e-12 \quad 4.222 e-12$ $9.177 e+00 \quad 7.297 e-12 \quad 1.179 e-11 \quad 5.989 e-12 \quad 7.086 e-12 \quad 4.040 e-12 \quad 4.689 e-12$ $9.665 e+00 \quad 6.802 e-12 \quad 1.286 e-11 \quad 6.145 e-12 \quad 7.311 e-12 \quad 4.562 e-12 \quad 5.272 e-12$ $\begin{array}{lllllll}1.012 e+01 & 6.561 e-12 & 1.413 e-11 & 6.934 e-12 & 7.472 e-12 & 5.217 e-12 & 5.974 e-12\end{array}$ $1.058 e+01 \quad 7.282 e-12 \quad 1.506 e-11 \quad 7.423 e-12 \quad 7.696 e-12 \quad 5.996 e-12 \quad 6.742 e-12$

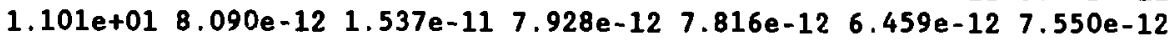
$1.155 e+018.528 e-12 \quad 1.629 e-11 \quad 9.875 e-12 \quad 7.916 e-12 \quad 7.089 e-12 \quad 8.329 e-12$ $1.199 e+01 \quad 8.638 e-12 \quad 1.765 e-11 \quad 1.132 e-11 \quad 8.029 e-12 \quad 7.660 e-12 \quad 9.050 e-12$ $1.250 e+018.844 e-12 \quad 1.897 e-11 \quad 1.139 e-11 \quad 8.084 e-12 \quad 8.011 e-12 \quad 9.646 e-12$ $1.307 e+01 \quad 9.157 e-12 \quad 2.060 e-11 \quad 1.243 e-11 \quad 8.138 e-12 \quad 8.330 e-12 \quad 1.018 e-11$ $1.354 e+01 \quad 9.431 e-12 \quad 2.183 e-11 \quad 1.318 e-118.173 e-128.584 e-12 \quad 1.063 e-11$ 


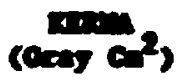

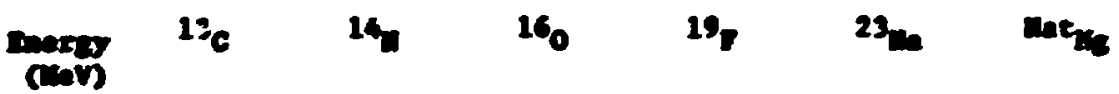

1.346e401 9.625e-12 $2.210 e-11 \quad 1.303 e-11 \quad 8.186 e-128.705-12$ 1.095e-1] 1.4130401 $9.760 e-12 \quad 2.220 e-11$ 1.419e-11 $8.201 e-12 \quad 8.810 e-12 \quad 1.115 e-11$ 1.441e+01 $9.802 e-12 \quad 2.263 \theta-11 \quad 1.465 e-11 \quad 8.283 e-12 \quad 8.981 e-12 \quad 1.121 e-11$ 1.46ee+01 $9.8430-12 \quad 2.310 e-11 \quad 1.515 e-11 \quad 8.376 e-12 \quad 9.168 e-12 \quad 1.1240-11$

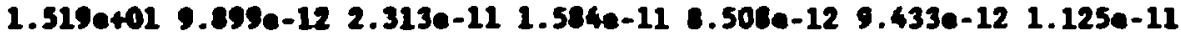
1.575a401 $9.916 a-12 \quad 2.277 \bullet-11 \quad 1.645 e-11 \quad 8.680 e-12 \quad 9.846 e-12 \quad 1.127 e-11$ $1.633 a+01 \quad 9.9340-12 \quad 2.235 a-11 \quad 1.717 a-11 \quad 8.863 a-12 \quad 1.015 a-11 \quad 1.124 e-11$ $1.692 e+029.951 e-12 \quad 2.244 e-11 \quad 1.774 e-11 \quad 9.012 e-12 \quad 1.055 e-11 \quad 1.117 e-11$ $1.752 e+01 \quad 1.000 a-11 \quad 2.257 e-11 \quad 1.798 e-11 \quad 9.153 e-12 \quad 1.113 e-11 \quad 1.104 e-11$ 1.813e+01 1.007e-11 2.267e-11 1.769e-11 9.291e-12 $1.168 e-11$ 1.077e-11 1.875et01 1.014e-11 2.316e-11 1.796e-11 9.400e-12 1.211e-11 1.055e-11 $2.000 e+01$ 1.025e-11 $2.427 e-11 \quad 1.903 e-11 \quad 9.513 e-12 \quad 1.250 e-11 \quad 1.025 e-11$ 


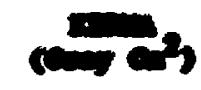

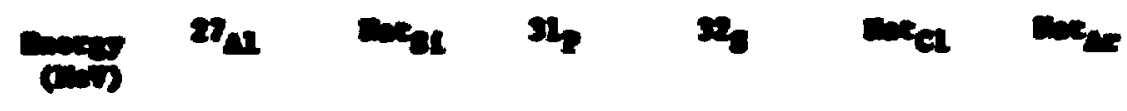

1.201e-1

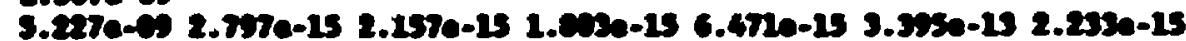

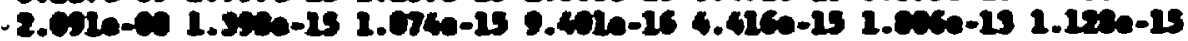

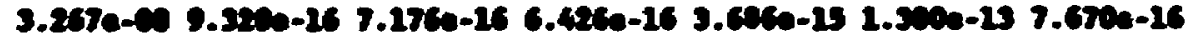

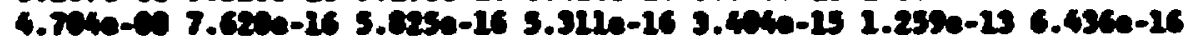

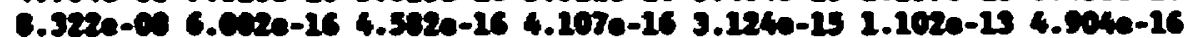
1.307e-07 4.675e-16 3.5710-16 3.157e-16 2.050-15 9.746e-14 3.6coe-16 1.002e-07 3.025e-16 2.922e-16 $2.5700-162.7300-15$ e.565e-14 3.115e-16 2.551e-07 3.235e-16 $2.5000-16$ 2.162e-16 2.625e-15 8.262e-14 2.640e-16 $3.345 e-072.002 e-16 \quad 2.1710-16$ 1.ec4e-16 2.543e-15 7.019e-14 2.262e-16 4.234e-07 2.472e-16 1.8see-16 1.63ee-16 2.479e-15 7.473e-14 2.015e-16 5.123e-07 2.224e-16 $1.715 e-16 \quad 1.459 e-16 \quad 2.426 e-15 \quad 7.203 e-14 \quad 1.791 e-16$ 7.527e-07 1.920e-16 $1.475 e-16$ 1.264e-16 $2.367 e-15$ 6.876e-14 1.546e-16 1.17Ge-06 $1.558 e-16$ 1.198e-16 $1.050 e-16$ 2.296e-15 $6.492 \mathrm{e}-14 \mathrm{1.280e-16}$ 1.511e-06 $1.316 e-16 \quad 1.024 e-16 \quad 8.952 e-17 \quad 2.200 e-15 \quad 6.273 e-14 \quad 1.123 e-16$ 2.091e-06 $1.141 e-16 \quad 8.972 e-17 \quad 7.804 e-17 \quad 2.279 e-15 \quad 6.046 e-14 \quad 9.552 e-17$ 2.741e-06 9.874e-17 $8.160 e-17 \quad 6.809 e-17 \quad 2.277 e-15 \quad 5.856 e-14 \quad 8.139 e-17$ 3.534e-06 8.699e-17 $7.581 e-17 \quad 6.053 e-17 \quad 2.274 e-15 \quad 5.716 e-14 \quad 7.076 e-17$ 4.704e-06 7.646e-17 6.998e-17 5.370e-17 2.271e-15 5.585e-14 6.136e-17 5.658e-06 6.856e-17 6.546e-17 4.868e-17 $2.268 e-15 \quad 5.492 e-14 \quad 5.437 e-17$ 6.737e-06 6.318e-17 6.134e-17 4.553e-17 $2.264 e-15$ 5.430e-14 4.957e-17 $8.321 e-065.802 e-175.677 e-17 \quad 4.296 e-17 \quad 2.260 e-15 \quad 5.384 e-14$ 4.491e-17 9.620e-06 5.387e-17 5.308e-17 4.129e-17 $2.258 e-15 \quad 5.327 e-14 \quad 4.106 e-17$

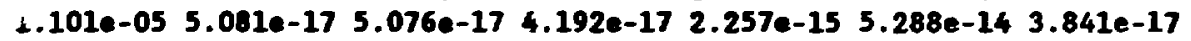

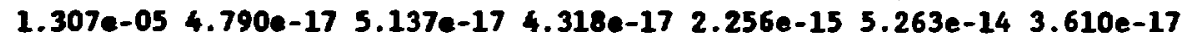
1.468e-05 4.554e-17 5.219e-17 4.453e-17 2.256e-15 5.236e-14 $3.412 e-17$ 1.581e-05 4.404e-17 5.280e-17 4.554e-17 2.255e-15 5.217e-14 $3.289 e-17$ $1.758 e-05 \quad 4.281 e-17 \quad 5.345 e-17 \quad 4.660 e-17 \quad 2.254 e-15 \quad 5.196 e-14 \quad 3.180 e-17$ $1.882 e-05 \quad 4.179 e-17 \quad 5.411 e-17 \quad 4.771 e-17 \quad 2.254 e-15 \quad 5.175 e-14 \quad 3.077 e-17$ 2.075e-05 4.077e-17 $5.482 e-17 \quad 4.887 e-17 \quad 2.253 e-15 \quad 5.153 e-14 \quad 2.986 e-17$ 2.277e-05 $3.992 e-17 \quad 5.570 e-17 \quad 5.032 e-17 \quad 2.252 e-15 \quad 5.137 e-14 \quad 2.887 e-17$ 2.417e-05 3.927e-17 5.646e-17 5.157e-17 2.251e-15 5.126e-14 2.312e-17 2.561e-05 $3.875 e-17 \quad 5.709 e-17 \quad 5.262 e-17 \quad 2.250 e-15 \quad 5.116 e-14 \quad 2.764 e-17$ 2.7i0e-05 $3.843 e-17 \quad 5.774 e-17 \quad 5.369 e-17 \quad 2.250 e-15 \quad 5.107 e-14 \quad 2.714 e-17$ 2.862e-05 3.818e-17 5.841e-17 5.480e-17 2.249e-15 5.097e-14 2.664e-17 $\begin{array}{lllllll}2.940 e-05 & 3.798 e-17 & 5.892 e-17 & 5.564 e-17 & 2.249 e-15 & 5.089 e-14 & 2.625 e-17\end{array}$ $\begin{array}{lllllll}3.099 e-05 & 3.778 e-17 & 5.945 e-17 & 5.651 e-17 & 2.249 e-15 & 5.082 e-14 & 2.590 e-17\end{array}$ $\begin{array}{lllllll}3.345 e-05 & 3.753 e-17 & 6.035 e-17 & 5.800 e-17 & 2.249 e-15 & 5.071 e-14 & 2.542 e-17\end{array}$ 3.601e-05 $3.746 e-17 \quad 6.147 e-17 \quad 5.984 e-17 \quad 2.249 e-15 \quad 5.059 e-14 \quad 2.487 e-17$

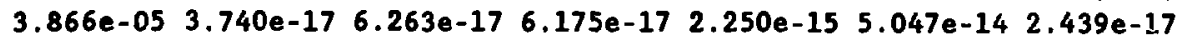
$\begin{array}{lllllll}4.048 e-05 & 3.736 e-17 & 6.362 e-17 & 6.339 e-17 & 2.250 e-15 & 5.036 e-14 & 2.402 e-17\end{array}$ 


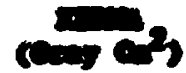

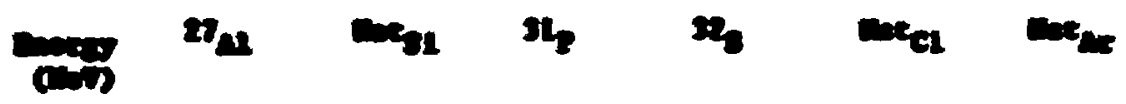

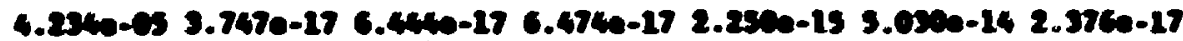

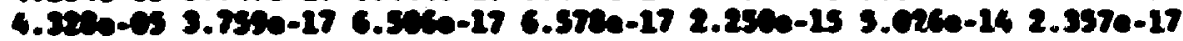

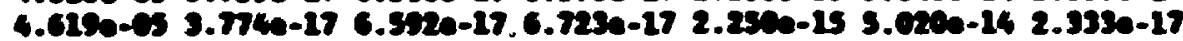
4.7170-6 3.750-17 6.675e-17 6.C6se-17 2.2500-15 5.015e-14 2.315e-17 4.0160-es 3.eve-17 $6.7450-17$ 6.sule-17 $2.2500-15$ 5.010e-14 $2.297 e-17$ 5.123e-es 3.0210-17 6.035e-17 7.133e-17 2.250e-15 5.0050-14 2.27ee-17 5.3320-05 3.0320-17 6.927e-17 7.205e-17 2.251e-15 5.000e-14 2.265e-17 5.654e-es 3.093e-17 7.06e-17 7.409e-17 2.251e-15 6.995e-14 2.249e-17 5.763e-05 3.927e-17 7.161e-17 7.650e-17 $2.251 e-15$ 4.950e-14 $2.236 e-17$ 6.097e-05 3.961e-17 7.240e-17 7.816e-17 2.251e-15 4.986e-14 2.222e-17 6.325e-05 4.005e-17 7.365e-17 8.027e-17 $2.251 e-15$ 4.980e-14 $2.205 e-17$ $6.557 e-05 \quad 4.047 e-17 \quad 7.467 e-17 \quad 0.199 e-17 \quad 2.251 e-15 \quad 4.977 e-14 \quad 2.195 e-17$ 6.674e-05 4.024e-17 7.545e-17 8.331e-17 2.251e-15 4.974e-14 2.192e-17 7.033e-05 4.133e-17 7.651e-17 6.510e-17 2.251e-15 4.970e-14 2.187e-17 7.155e-05 4.183e-17 7.758e-17 8.693e-17 2.252e-15 4.966e-14 2.181e-17 $7.527 e-05$ 4.234e-17 $7.868 e-17$ 8.881e-17 $2.252 e-15 \quad 4.963 e-14 \quad 2.176 e-17$ 7.780e-05 4.300e-17 8.007e-17 9.119e-17 2.252e-15 4.959e-14 2.169e-17 7.908e-05 4.339e-17 8.091e-17 9.265e-17 2.252e-15 4.956e-14 2.165e-17 $8.167 e-054.383 e-17 \quad 8.177 e-17 \quad 9.412 e-17 \quad 2.252 e-15$ 4.954e-14 2.164e-17 $8.431 e-05$ 4.447e-17 8.294e-17 9.612e-17 2.252e-15 4.951e-14 2.166e-17 8.834e-05 4.528e-17 8.442e-17 9.866e-17 2.253e-15 4.948e-14 2.168e-17 9.108e-05 4.611e-17 8.592e-17 1.012e-16 2.253e-15 4.945e-14 2.171e-17 9.386e-05 4.679e-17 8.715e-17 $1.033 e-16 \quad 2.253 e-15 \quad 4.942 e-14 \quad 2.173 e-17$ $9.668 e-05 \quad 4.748 e-17 \quad 8.840 e-17 \quad 1.055 e-16 \quad 2.253 e-15 \quad 4.940 e-14 \quad 2.175 e-17$ 9.811e-05 4.800e-17 8.934e-17 1.071e-16 2.253e-15 4.939e-14 2.177e-17 1.025e-04 4.874e-17 9.066e-17 $1.093 e-16 \quad 2.254 e-15 \quad 4.937 e-14 \quad 2.180 e-17$ 1.099e-04 5.045e-17 $9.354 e-17 \quad 1.138 e-16 \quad 2.254 e-15 \quad 4.935 e-14 \quad 2.194 e-17$ 1.176e-04 5.269e-17 9.728e-17 $1.196 e-16 \quad 2.254 e-15 \quad 4.932 e-14 \quad 2.216 e-17$ 1.256e-04 5.500e-17 1.012e-16 i.256e-16 2.255e-15 4.929e-14 $2.239 e-17$ $1.338 e-045.742 e-171.051 e-16 ; 319 e-162.255 e-15 \quad 4.926 e-142.265 e-17$ 1.406e-04 5.971e-17 $1.088 e-16$ i.377e-16 2.256e-15 4.924e-14 2.296e-17 1.511e-04 6.234e-17 $1.131 e-16 \quad 1.443 e-16 \quad 2.257 e-15 \quad 4.920 e-14 \quad 2.333 e-17$ 1.601e-04 6.532e-17 1.179e-16 1.518e-16 2.259e-15 4.917e-14 2.385e-17 $\begin{array}{lllllll}1.694 e-04 & 6.815 e-17 & 1.224 e-16 & 1.588 e-16 & 2.261 e-15 & 4.913 e-14 & 2.442 e-17\end{array}$ 1.789e-04 7.110e-17 $1.270 e-16 \quad 1.661 e-16 \quad 2.263 e-15 \quad 4.909 e-14 \quad 2.501 e-17$ 1.887e-04 7.412e-17 $1.318 e-16 \quad 1.736 e-16 \quad 2.265 e-15 \quad 4.905 e-14 \quad 2.563 e-17$ 1.988e-04 7.723e-17 $1.367 e-16 \quad 1.813 e-16 \quad 2.266 e-15 \quad 4.901 e-14 \quad 2.630 e-17$ 2.091e-04 8.044e-17 $1.417 e-16 \quad 1.892 e-16 \quad 2.268 e-15 \quad 4.898 e-14 \quad 2.698 e-17$ 2.741e-04 9.246e-17 1.603e-16 2.184e-16 2.276e-15 4.894e-14 2.954e-17 3.267e-04 $1.114 e-16 \quad 1.893 e-16 \quad 2.640 e-16 \quad 2.287 e-15 \quad 4.920 e-14 \quad 3.370 e-17$ $3.811 e-04 \quad 1.288 e-162.156 e-16 \quad 3.054 e-16 \quad 2.297 e-15 \quad 4.999 e-14 \quad 3.763 e-17$ 

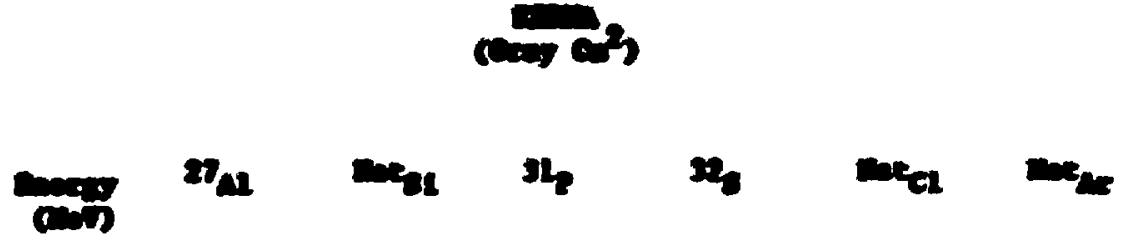

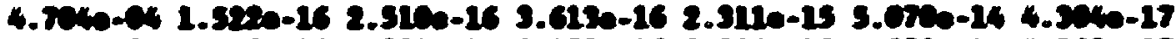
4.9010-4 1.7160-16 2.6010-16 4.0720-16 2.3220-15 4.9030-14 4.750-17

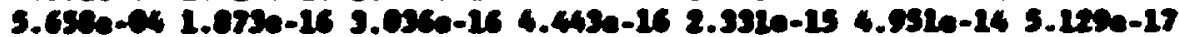

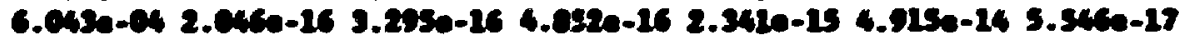

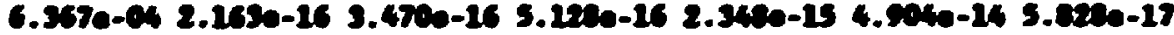
7.156-04 2.347e-16 3.7440-16 5.5010-16 2.359e-15 4.9020-14 6.2760-17 6.3220-94 2.6710-16 4.226e-16 6.3230-16 2.3770-15 4.042e-14 7.075 -17 $9.1770-043.005 e-164.7240-16$ 7.210e-16 $2.395 e-15$ 4.e97e-14 7.914e-17 1.05e-03 $3.302 e-165.242 e-167.992 e-162.425 e-15$ 4.09se-14 8.069e-17 $1.307 e-03$ 4.039e-16 $6.2450-169.509 e-162.4910-15$ 4.904e-14 $1.055 e-16$ 1.501e-03 4.923e-16 $7.539 e-16$ 1.155e-15 $2.455 e-15$ 4.90ee-14 1.267e-16 1.ee2e-03 5.896e-16 8.962e-16 1.379e-15 2.492e-15 4.917e-14 1.551e-16 2.204e-03 6.957e-16 1.052e-15 1.624e-15 2.536e-15 4.916e-14 1.849e-16 2.561e-03 e.106e-16 $1.220 e-15$ 1.889e-15 $2.585 e-15 \quad 4.931 e-14 \quad 2.183 e-16$ $2.940 e-03 \quad 9.343 e-161.401 e-15 \quad 2.174 e-15$ 2.645e-15 4.950e-14 2.555e-16 $3.345 e-03 \quad 1.065 e-15 \quad 1.595 e-15 \quad 2.480 e-15 \quad 2.709 e-15 \quad 4.963 e-142.970 e-16$ 3.777e-03 1.199e-15 1.802e-15 2.807e-15 2.778e-15 4.958e-14 3.429e-16 4.234e-03 1.340e-15 2.022e-15 3.153e-15 2.855e-15 $5.020 e-14$ 3.936e-16 $5.763 e-031.729 e-15 \quad 2.514 e-15 \quad 3.929 e-15 \quad 3.027 e-15 \quad 5.039 e-14 \quad 5.148 e-16$ $7.527 e-03 \quad 3.941 e-15 \quad 3.291 e-15 \quad 5.732 e-15$ 3.350e-15 $5.073 e-147.362 e-16$ 1.025e-02 2.846e-15 4.166e-15 8.812e-15 $3.864 e-15 \quad 5.485 e-14 \quad 1.082 e-15$ $1.511 \bullet-02 \quad 3.812 e-15 \quad 5.268 e-15 \quad 1.196 e-14 \quad 4.889 a-15$ 5.560e-14 $1.852 e-15$ 2.091e-02 3.968e-15 6.597e-15 1.420e-14 5.344e-15 $5.689 e-14 \quad 3.263 e-15$ 2.646e-02 3.864e-15 8.047e-15 1.711e-14 5.493e-15 7.016e-14 4.565e-15 3.267e-02 $1.777 e-14 \quad 9.657 e-15 \quad 2.300 e-14 \quad 7.470 e-15 \quad 6.569 e-14 \quad 5.635 e-15$ $3.953 e-021.782 e-13 \quad 1.160 e-14 \quad 3.077 e-14 \quad 6.412 e-15 \quad 6.342 e-14 \quad 6.811 e-15$ 4.704e-02 4.854e-14 $1.454 e-14 \quad 3.833 e-14 \quad 6.917 a-15 \quad 6.791 e-14 \quad 8.091 e-15$ 5.761e-02 3.043e-14 $1.943 e-14 \quad 4.499 e-14 \quad 7.568 e-15 \quad 7.237 e-14 \quad 9.678 e-15$ 7.002e-02 2.664e-14 3.021e-14 5.319e-14 8.977e-15 7.869e-14 $1.166 e-14$ 8.322e-02 8.836e-14 $3.113 e-14 \quad 6.258 e-14 \quad 2.081 e-14 \quad 8.143 e-14 \quad 1.377 e-14$ 9.891e-02 2.944e-13 2.576e-14 6.747e-14 6.027e-14 8.162e-14 $1.608 e-14$ $1.307 e-01 \quad 9.922 e-14 \quad 2.075 e-14 \quad 5.283 e-14 \quad 2.450 e-13 \quad 8.745 e-14 \quad 1.990 e-14$ 1.819e-01 2.643e-13 7.181e-14 7.765e-14 1.206e-13 $1.044 e-13 \quad 2.738 e-14$ $2.075 e-01 \quad 1.959 e-13 \quad 5.269 e-13 \quad 1.053 e-13 \quad 1.226 e-13 \quad 1.397 e-13 \quad 3.452 e-14$ 2.417e-01 2.316e-13 4.102e-13 9.491e-14 $1.099 e-13 \quad 1.257 e-13 \quad 4.018 e-14$ 2.710e-01 $1.322 e-13 \quad 3.192 e-13 \quad 1.28 .5 e-13 \quad 9.829 e-14 \quad 1.321 e-13 \quad 4.623 e-14$ $2.940 e-01 \quad 3.036 e-13 \quad 2.952 e-13 \quad 1.409 e-13 \quad 1.744 e-13 \quad 1.539 e-13 \quad 5.135 e-14$ 3.345e-01 2.977e-13 2.873e-13 $1.128 \mathrm{e}-13 \quad 1.200 \mathrm{e}-13 \quad 1.509 \mathrm{e}-13 \quad 5.488 \mathrm{e}-14$ 3.776e-01 2.940e-13 $2.889 e-13 \quad 2.167 e-13 \quad 8.76 \mathrm{Ce}-14 \quad 1.862 e-13 \quad 4.815 e-14$ 4.234e-01 3.129e-13 2.984e-13 $1.678 e-13 \quad 2.076 e-13 \quad 2.035 e-13 \quad 4.002 e-14$ 5.123e-01 4.108e-13 $3.099 e-13 \quad 2.784 e-13 \quad 1.574 e-13 \quad 2.071 e-13 \quad 5.501 e-14$ 


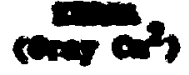

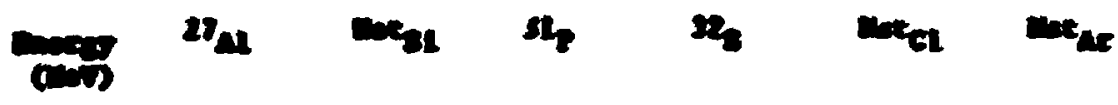

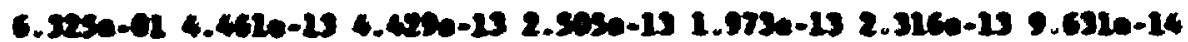
7.5270-01 4.640-13 4.694-13 2.400-13 2.059e-13 2.6920-13 $1.2010-13$

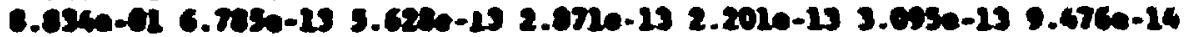

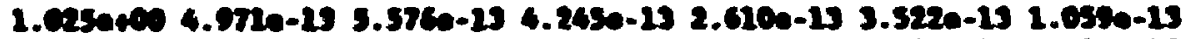

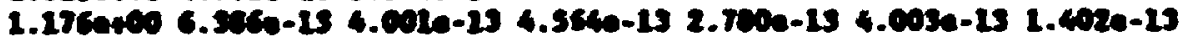

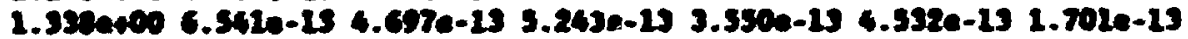

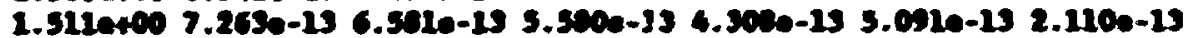
$1.6940+407.6740-13 \quad 0.9360-13 \quad 6.2530-134.7420-135.7720-132.0170-13$ $1.0070+60$ e.343e-13 0.9210-13 $7.0210-13 \quad 6.0070-13 \quad 6.475 e-132.7040-13$ $2.05104001 .025 e-12 \quad 9.0540-137.719 e-13 \quad 1.052 e-12 \quad 6.919 e-13 \quad 3.0600-13$ $2.305 e+001.030 e-12 \quad 9.3940-13 \quad 0.313 e-13 \quad 1.1010-12 \quad 7.520 e-13 \quad 3.333 e-13$ $2.530 e+009.350 e-13 \quad 1.007 e-12 \quad 9.037 e-13 \quad 1.324 e-12 \quad 0.391 e-13 \quad 3.626 e-13$ $2.741 e+001.103 e-12 \quad 1.003 e-12 \quad 1.127 e-12 \quad 1.590 e-12 \quad 9.252 e-13 \quad 3.941 e-1.3$ $3.0110+001.134 e-12 \quad 1.005 e-12 \quad 1.200 e-12 \quad 2.307 e-12 \quad 1.018 e-12 \quad 4.158 e-13$ $3.267 e+00$ 1.181e-12 $1.061 e-12 \quad 1.354 e-12 \quad 2.555 e-12 \quad 1.109 e-12 \quad 4.332 e-13$ $3.534 e+00 \quad 1.230 e-12 \quad 1.074 e-12 \quad 1.231 e-12 \quad 2.791 e-12 \quad 1.218 e-12 \quad 4.586 e-13$ $3.811 e+001.317 e-12 \quad 9.478 e-13 \quad 1.470 e-12 \quad 3.452 e-12 \quad 1.351 e-12 \quad 4.912 e-13$ $4.069 e+00 \quad 1.313 e-12 \quad 1.231 e-12 \quad 1.517 e-12 \quad 3.950 e-12 \quad 1.501 e-12 \quad 5.244 e-13$ $4.396 e+001.377 e-12 \quad 1.363 e-12 \quad 1.606 e-12 \quad 3.334 e-12 \quad 1.675 e-32 \quad 5.674 e-13$ $4.704 e+001.432 e-12 \quad 1.575 e-12 \quad 1.627 e-12 \quad 3.220 e-12 \quad 1.869 e-12 \quad 6.009 e-13$ $4.991 e+00 \quad 1.540 e-12 \quad 1.790 e-12 \quad 1.637 e-12 \quad 3.308 e-12 \quad 2.057 e-12 \quad 6,288 e-13$ $5.352 e+00 \quad 1.686 e-12 \quad 1.677 e-12 \quad 1.585 e-12 \quad 3.412 e-12 \quad 2.264 e-12 \quad 6.675 e-13$

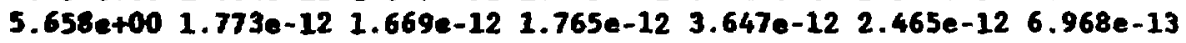
$6.042 e+001.971 e-12 \quad 2.096 e-12 \quad 1.861 e-12 \quad 4.110 e-12 \quad 2.677 e-12 \quad 7.193 e-13$ $6.367 e+002.062 e-12 \quad 2.280 e-12 \quad 2.064 e-12 \quad 4.519 e-12 \quad 2.908 e-12 \quad 7.477 e-13$ $6.737 e+002.246 e-12 \quad 2.723 e-12 \quad 2.320 e-12 \quad 4.760 e-12 \quad 3.10 B e-12 \quad 7.832 e-13$ $7.156 e+00 \quad 2.455 e-12 \quad 3.437 e-12 \quad 2.574 e-12 \quad 5.019 e-12 \quad 3.318 e-12 \quad 8.241 e-13$ $7.548 e+00 \quad 2.638 e-12 \quad 4.199 e-12 \quad 2.839 e-12 \quad 5.328 e-12 \quad 3.511 e-12 \quad 8.644 e-13$ $7.910 e+00 \quad 2.937 e-12 \quad 5.362 e-12 \quad 3.115 e-12 \quad 5.619 e-12 \quad 3.678 e-12 \quad 9.014 e-13$ B.322e+00 $3.155 e-12 \quad 5.502 e-12 \quad 3.430 e-12 \quad 5.924 e-12 \quad 3.834 e-12 \quad 9.411 e-13$ 8.787e+00 3.504e-12 6.335e-12 $3.844 \theta-12 \quad 6.282 e-12 \quad 3.986 e-12 \quad 9.901 e-13$ $9.177 e+00 \quad 3.805 e-12 \quad 7.260 e-12 \quad 4.262 e-12 \quad 6.633 e-12 \quad 4.131 e-12 \quad 1.038 e-12$ $9.665 e+00 \quad 4.252 e-12 \quad 8.059 e-12 \quad 4.559 e-12 \quad 7.000 e-12 \quad 4.292 e-12 \quad 1.093 e-12$ $1.012 e+01 \quad 4.736 e-12 \quad B .880 e-12 \quad 4.859 e-12 \quad 7.404 e-12 \quad 4.421 e-12 \quad 1.154 e-12$ $1.058 e+01 \quad 5.188 e-12 \quad 9.629 e-12 \quad 5.118 e-12 \quad 7.786 e-12 \quad 4.593 e-12 \quad 1.212 e-12$ $1.101 e+01 \quad 5.703 e-12 \quad 1.023 e-11 \quad 5.354 e-12 \quad 8.156 e-12 \quad 4.786 e-12 \quad 1.271 e-12$ $1.155 e+01 \quad 6.277 e-12 \quad 1.081 e-11 \quad 5.612 e-12 \quad 8.403 e-12 \quad 4.985 e-12 \quad 1.336 e-12$ $1.199 e+01 \quad 6.775 e-12 \quad 1.133 e-11 \quad 5.875 e-12 \quad 8.524 e-12 \quad 5.177 e-12 \quad 1.402 e-12$

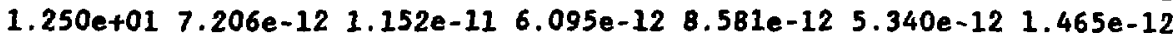

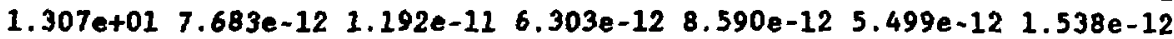
$1.354 e+01 \quad 8.102 e-12 \quad 1.229 e-11 \quad 6.496 e-12 \quad 8.594 e-12 \quad 5.655 e-12 \quad 1.605 e-12$ 


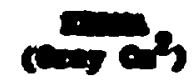

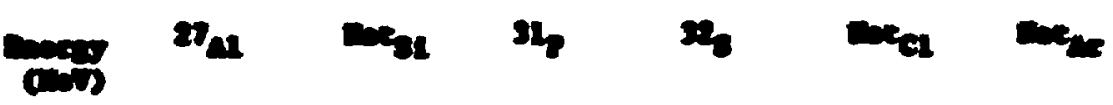

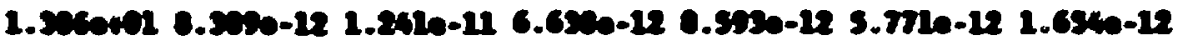

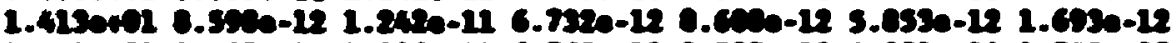

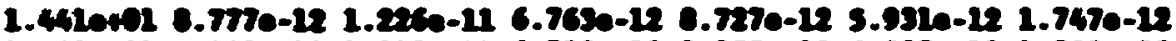

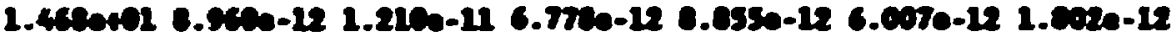

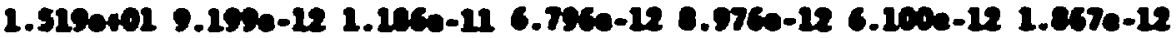
1.5750401 9.46e-12 1.150-11 6.015e-12 9.245e-12 6.146-12 1.937e-12

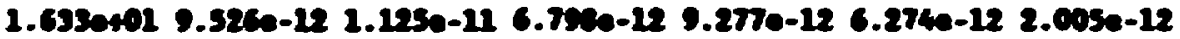
$1.69204019 .4750-12$ 1.106e-11 $6.7070-129.2970-12 \quad 6.3450-12$ 2.064e-12 1.7520+01 9.316e-12 $1.0920-116.6310-129.5600-12 \quad 6.401 e-12$ 2.121e-12 $1.0130+019.039 e-12$ 1.059e-11 $6.610 e-129.0510-12 \quad 6.428 e-12$ 2.175e-12 1.675a401 $0.9200-12 \quad 1.022 a-11 \quad 6.576 e-12 \quad 1.016 a-11 \quad 6.512 e-12 \quad 2.236 e-12$ 2.000e+01 8.654e-12 $9.281 e-12 \quad 6.483 e-12 \quad 1.072 e-11 \quad 6.602 e-12 \quad 2.319 e-12$ 

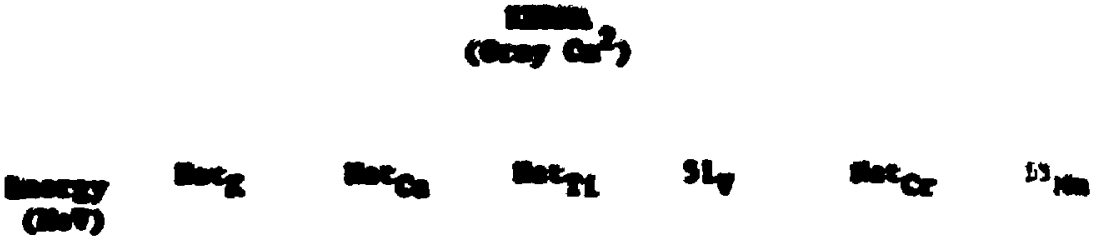

\begin{abstract}
$1.3010-1$
5.227e-1 $1.2260-142.020-152.7150-141.340-141.400-14$ 4.029e-14

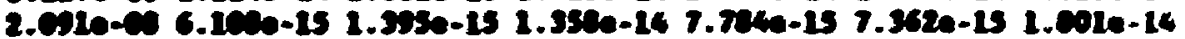

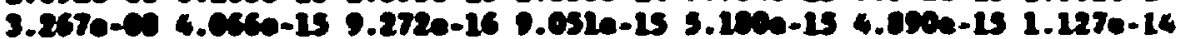
4.706e-C 3.3150-15 7.623e-16 7.405e-15 4.2360-15 3.9820-15 9.376e-15 e.322e-ce 2.5n2e-15 s.97e-16 5.026e-15 3.335e-15 3.120e-15 7.318e-15 $1.307 e-072.0040-15$ 4.635e-26 4.530e-15 2.597e-15 $2.4170-15$ 5.5710-15 1.e62e-07 1.6see-15 3.012e-16 3.702e-15 2.127e-15 1.901e-15 4.637e-15 2.561e-07 1.376e-15 3.235e-26 3.132e-15 $1.796 e-15$ 1.6610-15 3.877e-15 3.345e-07 1.167e-15 2.793a-16 2.715e-15 1.559a-15 1.44e-15 3.129e-15 4.234e-07 $1.044 e-15$ 2.470e-16 2.395e-15 $1.375 e-15$ 1.261e-15 2.695e-15 5.123a-07 9.36e-16 2.221e-16 2.155e-15 $1.237 e-15$ 1.151e-15 $2.542 e-15$ 7.527e-07 0.059e-16 $1.915 e-16 \quad 1.860 e-15$ l.068e-15 $9.934 e-16 \quad 2.281 e-15$ $1.176 e-06$ 6.506e-16 1.552e-16 1.509e-15 $8.650 e-16 \quad 8.107 e-16$ 1.823e-15 1.511e-06 5.470e-16 $1.310 e-16 \quad 1.273 e-15 \quad 7.286 e-16 \quad 7.089 e-16 \quad 1.572 e-15$ 2.091e-0s 4.721e-16 $1.135 e-16 \quad 1.102 e-15 \quad 6.304 e-16 \quad 6.043 e-16 \quad 1.436 e-15$ 2.741e-05 4.059e-16 9.823e-17 9.504e-16 5.441e-16 5.144e-16 1.293e-15 $3.534 e-06 \quad 3.552 e-16 \quad 8.669 e-17 \quad 8.341 e-16 \quad 4.773 e-16 \quad 4.475 e-16 \quad 1.126 e-15$ 4.704e-06 3.095e-16 7.646e-17 7.289e-16 4.173e-16 3.904e-16 9.360e-16 5.658e-06 2.751e-16 6.933e-17 $6.494 e-16 \quad 3.721 e-16 \quad 3.486 e-16 \quad 8.585 e-16$ 6.737e-06 2.515e-16 $6.432 e-17 \quad 5.943 e-16 \quad 3.406 e-16 \quad 3.195 e-16 \quad 7.998 e-16$ $8.321 e-06 \quad 2.282 e-16 \quad 5.942 e-17 \quad 5.401 e-16 \quad 3.099 e-16 \quad 2.904 e-16 \quad 7.229 e-16$ 9.620e-06 2.089e-16 5.556e-17 4.952e-16 $2.845 e-16 \quad 2.655 e-16 \quad 6.396 e-16$ $1.101 e-05 \quad 1.950 e-16 \quad 5.240 e-17 \quad 4.625 e-16 \quad 2.660 e-16 \quad 2.499 e-16 \quad 5.796 e-16$ $1.307 e-05 \quad 1.803 e-16 \quad 4.880 e-17 \quad 4.291 e-16 \quad 2.474 e-16 \quad 2.375 e-16 \quad 5.607 e-16$ 1.468e-05 1.687e-16 4.570e-17 4.005e-16 2.315e-16 $2.245 e-16 \quad 5.433 e-16$ $1.581 e-05 \quad 1.611 e-16 \quad 4.391 e-17 \quad 3.827 e-16 \quad 2.216 e-16 \quad 2.148 e-16 \quad 5.303 e-16$ 1.758e-05 $1.544 e-16 \quad 4.227 e-17 \quad 3.666 e-16 \quad 2.127 e-16 \quad 2.046 e-16 \quad 5.165 e-16$

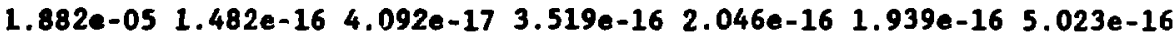
2.075e-05 $1.425 e-16 \quad 3.969 e-17 \quad 3.384 e-16 \quad 1.972 e-16 \quad 1.834 e-16 \quad 4.873 e-16$ 2.277e-05 $1.365 e-16 \quad 3.868 e-17 \quad 3.237 e-16 \quad 1.892 e-16 \quad 1.764 e-16 \quad 4.685 e-16$ 2.417e-05 $1.319 e-16 \quad 3.807 e-17 \quad 3.126 e-16 \quad 1.83+3-16 \quad 1.717 e-16 \quad 4.523 e-16$ 2.561e-05 $1.284 e-16 \quad 3.762 e-17 \quad 3.043 e-16 \quad 1.787 e-16 \quad 1.677 e-16 \quad 4.388 e-16$ 2.710e-05 $1.253 e-16 \quad 3.718 e-17 \quad 2.965 e-16 \quad 1.744 e-16 \quad 1.637 e-16 \quad 4.250 e-16$ 2.862e-05 $1.222 e-16 \quad 3.689 e-17 \quad 2.892 e-16 \quad 1.706 e-16 \quad 1.595 e-16 \quad 4.107 e-16$ 2.940e-05 $1.201 e-16 \quad 3.670 e-17 \quad 2.840 e-16 \quad 1.678 e-16 \quad 1.564 e-16 \quad 3.998 e-16$ 3.099e-05 $1.181 e-16 \quad 3.649 e-17 \quad 2.790 e-16 \quad 1.650 e-16 \quad 1.534 e-16 \quad 3.886 e-16$ 3.345e-05 $1.150 e-16 \quad 3.622 e-17 \quad 2.713 e-16 \quad 1.610 e-16 \quad 1.498 e-16 \quad 3.695 e-16$ 3.601e-05 $1.115 e-16 \quad 3.605 e-17 \quad 2.626 e-16 \quad 1.564 e-16 \quad 1.456 e-16 \quad 3.457 e-16$

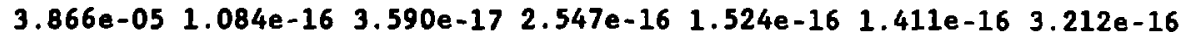
$4.048 e-051.059 e-16 \quad 3.590 e-17 \quad 2.486 e-16 \quad 1.491 e-16 \quad 1.373 e-16 \quad 3.005 e-16$
\end{abstract}




\section{$\sin 2$}

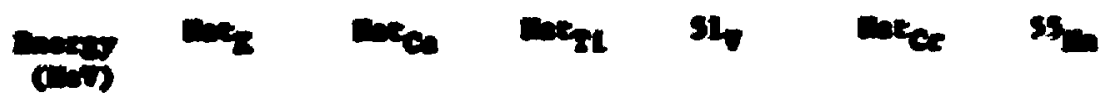

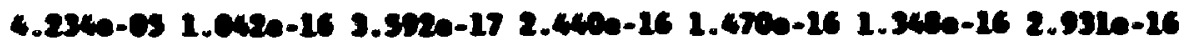
4.200-63 1.026-16 3.5930-17 2.407e-16 :.4850-16 1.3310-16 2.502e-16

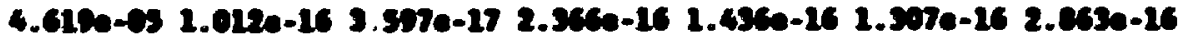

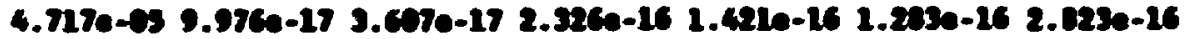

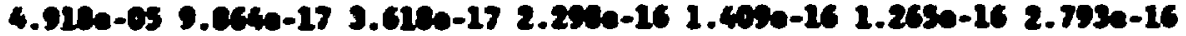
5.123e-c5 9.730e-17 3.633e-17 2.262e-16 $1.393 e-16 \quad 1.242 e-16 \quad 2.7510-16$ 5.3320-05 $9.6030-17$ 3.c4e-17 $2.2210-16 \quad 1.3760-16 \quad 1.230 e-162.7100-16$ 5.654e-05 9.454e-17 3.677e-17 $2.106 e-16$ 1.362e-16 1.216e-16 2.657e-16 $5.7630-05 \quad 9.347 e-17 \quad 3.703 e-17 \quad 2.150-16 \quad 1.351 e-16 \quad 1.205 e-16 \quad 2.616 e-16$ 6.037e-05 9.240e-17 3.729e-17 2.129e-16 $1.340 e-16 \quad 1.1940-16 \quad 2.570 e-16$ 6.325e-05 9.123e-17 3.765e-17 2.056e-16 $1.3260-16$ 1.161e-16 2.516e-16 $6.557 e-05$ 9.034e-17 3.601e-17 2.071e-16 $1.317 e-16$ 1.172e-16 2.46ee-16 6.674e-05 $\quad .967 e-17$ 3.826e-17 $2.052 e-16 \quad 1.312 e-16 \quad 1.164 e-16 \quad 2.433 e-16$ 7.033e-05 8.894e-17 3.866e-17 2.029e-16 $1.305 e-16 \quad 1.154 e-16 \quad 2.385 e-16$ 7.155e-05 $8.821 e-17 \quad 3.904 e-17 \quad 2.008 e-16 \quad 1.298 e-16 \quad 1.145 e-16 \quad 2.338 e-16$ $7.527 e-05 \quad 8.752 e-17 \quad 3.945 e-17 \quad 1.987 e-16 \quad 1.291 e-16 \quad 1.136 e-16 \quad 2.289 e-16$ $7.780 e-05 \quad 8.678 e-17 \quad 4.003 e-17 \quad 1.963 e-16 \quad 1.283 e-16 \quad 1.125 e-16 \quad 2.234 e-16$ 7.908e-05 8.635e-17 4.039e-17 $1.949 e-16 \quad 1.281 e-16 \quad 1.118 e-16 \quad 2.215 e-16$ $8.167 e-05 \quad 8.592 e-17 \quad 4.075 e-17 \quad 1.936 e-16 \quad 1.278 e-16 \quad 1.112 e-16 \quad 2.198 e-16$ 8.431e-05 8.546e-17 4.125e-17 $1.920 e-16 \quad 1.274 e-16 \quad 1.107 e-16 \quad 2.176 e-16$ 8.834e-05 8.492e-17 4.189e-17 1.901e-16 $1.272 e-16 \quad 1.101 e-16 \quad 2.149 e-16$ 9.108e-05 8.440e-17 4.259e-17 $1.882 e-16 \quad 1.271 e-16 \quad 1.095 e-16 \quad 2.122 e-16$ $9.386 e-05$ 8.409e-17 4.318e-17 $1.869 e-16$ 1.271e-16 $1.091 e-16 \quad 2.100 e-16$ 9.668e-05 8.379e-17 4.377e-17 $1.857 e-16 \quad 1.270 e-16 \quad 1.087 e-16 \quad 2.078 e-16$ 9.811e-05 8.356e-17 4.422e-17 $1.848 e-16 \quad 1.270 e-16 \quad 1.084 e-16 \quad 2.062 e-16$ $1.025 e-04 \quad 8.333 e-17 \quad 4.484 e-17 \quad 1.837 e-16 \quad 1.271 e-16 \quad 1.081 d-16 \quad 2.050 e-16$ 1.099e-04 8.297e-17 4.619e-17 $1.818 e-16 \quad 1.283 e-16 \quad 1.084 e-16 \quad 2.084 e-16$ 1.176e-04 8.270e-17 4.794e-17 $1.797 e-16 \quad 1.300 e-16 \quad 1.089 e-16 \quad 2.139 e-16$ $1.256 e-04 \quad 8.261 e-17 \quad 4.981 e-17 \quad 1.780 e-16 \quad 1.324 e-16 \quad 1.095 e-16 \quad 2.200 e-16$ 1. $338 e-04 \quad 8.271 e-17 \quad 5.179 e-17 \quad 1.767 e-16 \quad 1.368 e-16 \quad 1.100 e-16 \quad 2.269 e-16$ 1.406e-04 8.296e-17 5.364e-17 $1.758 e-16 \quad 1.437 e-16 \quad 1.105 e-16 \quad 2.337 e-16$ 1.511e-04 8.337e-17 5.583e-17 $1.752 e-16 \quad 1.614 e-16 \quad 1.111 e-16 \quad 2.421 e-16$ 1.601e-04 8.400e-17 5.831e-17 1.753e-16 2.295e-16 1.117e-16 2.571e-16 1.694e-04 8.472e-17 6.070e-17 1.759e-16 $1.347 e-15 \quad 1.124 e-16 \quad 2.761 e-16$ 1.789e-04 8.560e-17 $6.317 e-17 \quad 1.765 e-16 \quad 4.429 e-16 \quad 1.130 e-16 \quad 2.973 e-16$ 1.887e-04 8.658e-17 6.572e-17 $1.771 e-16 \quad 1.902 e-16 \quad 1.136 e-16 \quad 3.236 e-16$ $1.988 e-04 \quad 8.770 e-17 \quad 6.839 e-17 \quad 1.777 e-16 \quad 1.583 e-16 \quad 1.143 e-16 \quad 3.572 e-16$ 2.091e-04 8.933e-17 $7.114 e-17 \quad 1.783 e-16 \quad 1.514 e-16 \quad 1.156 e-16 \quad 4.003 e-16$ 2.741e-04 9.792e-17 $8.148 e-17 \quad 1.842 e-16 \quad 1.566 e-16 \quad 1.238 e-16 \quad 1.193 e-15$ 3.267e-04 $1.113 e-16 \quad 9.791 e-17 \quad 1.965 e-16 \quad 1.787 e-16 \quad 1.366 e-16 \quad 1.022 e-14$ 3.811e-04 1.235e-16 $1.131 e-16 \quad 2.078 e-16 \quad 1.989 e-16 \quad 1.482 e-16 \quad 3.153 e-14$ 


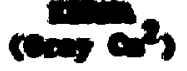

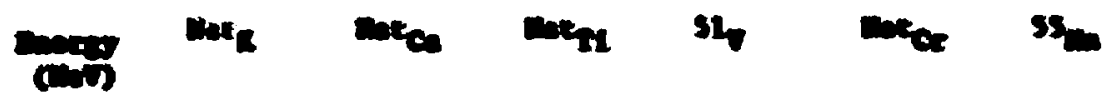

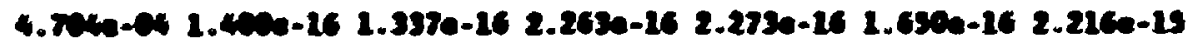

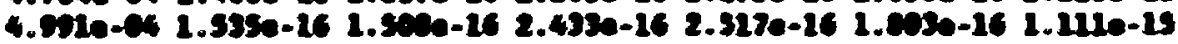

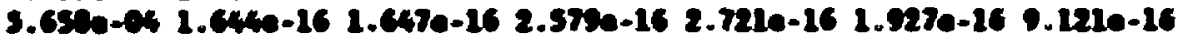

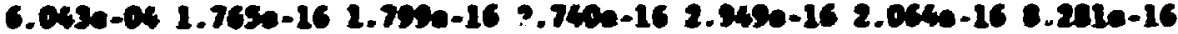
$6.357 a-04$ 1.047a-16 1.503a-16 $2.0450-16$ 3.105e-16 $2.173 a-168.460-16$ 7.256-04 $1.9740-16$ 2.05e-16 3.0240-16 3.354e-16 2.344e-16 8.643e-16

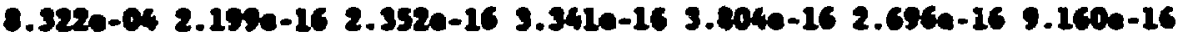
$9.177 e-042.432 e-162.6490-163.675 e-164.320 e-162.982 e-16$ 16.094e-15 1.0560-03 2.706e-16 2.5120-16 4.0520-16 4.985e-16 3.293e-16 2.927e-15 $1.307 e-03 \quad 3.3540-16 \quad 3.570 e-16 \quad 4.775 e-16$ 6.516e-16 4.05ee-16 7.614e-15 $1.5010-034.3690-16 \quad 4.3940-16 \quad 5.760 e-16 \quad 9.346 e-16 \quad 5.2160-16 \quad 1.604 e-15$ 1.002e-03 5.639e-16 5.144a-16 $\quad 6.910 e-16 \quad 1.029 e-15 \quad 6.505 e-16 \quad 5.165 e-15$ 2.203e-03 7.437e-16 $\quad 6.072 e-16 \quad 6.364 e-16$ 1.271e-15 $7.551 e-16$ 2.761e-14 2.561e-03 1.053e-15 7.051e-16 1.08ee-15 1.699e-15 $9.345 e-16 \quad 7.115 e-14$ $2.940 e-03 \quad 1.481 e-15 \quad 8.126 e-16 \quad 1.764 e-15 \quad 2.513 e-15 \quad 1.263 e-15 \quad 3.354 e-14$ 3.345e-03 $1.742 e-15 \quad 9.230 e-165.375 e-15$ 4.526e-15 $1.871 e-15$ 1.179e-14 3.777e-03 1.536e-15 1.033e-15 3.905e-15 $1.307 e-14 \quad 3.240 e-15 \quad 6.495 e-15$ 4.234e-03 1.316e-15 $1.150 e-15 \quad 5.568 e-15 \quad 6.742 e-14 \quad 5.771 e-15 \quad 4.416 e-15$ 5.763e-03 2.196e-15 $1.417 e-15 \quad 3.784 e-15 \quad 4.173 e-14 \quad 7.352 e-15 \quad 2.688 e-15$ $7.527 e-032.199 e-151.837 e-15$ 6.515e-15 $5.643 e-14 \quad 8.051 e-15 \quad 2.007 e-14$ 1.025e-02 4.784e-15 $2.390 e-15 \quad 1.557 e-14 \quad 1.983 e-14 \quad 7.974 e-15 \quad 2.406 e-14$ 1.511e-02 5.218e-15 $3.220 e-15 \quad 5.442 e-14 \quad 7.014 e-14 \quad 4.630 e-15 \quad 4.765 e-15$ 2.091e-02 5.106e-15 4.296e-15 $1.477 e-13 \quad 4.266 e-14 \quad 3.854 a-15$ 1.180e-14 2.646e-02 1.237e-14 6.152e-15 9.406e-14 3.403e-14 4.851e-15 2.656e-14 3.267e-02 7.132e-15 5.898e-15 $4.324 e-14 \quad 2.064 e-14 \quad 7.971 e-15 \quad 1.567 e-14$ 3.953e-02 6.738e-15 6.900e-15 5.356e-14 $1.029 e-14 \quad 8.544 e-15 \quad 5.407 e-14$ 4.704e-02 2.978e-14 7.864e-15 3.173e-14 $1.400 e-14$ 6.186e-15 2.471e-14 5.761e-02 1.002e-14 $1.041 e-14 \quad 7.378 e-14 \quad 2.016 e-14 \quad 3.428 e-14 \quad 1.494 e-14$ 7.002e-02 4.949e-14 8.654e-15 4.585e-14 6.011e-14 $1.556 e-14 \quad 3.589 e-14$ 8.322e-02 2.441e-14 $1.071 e-14 \quad 2.954 e-14 \quad 3.306 e-14 \quad 1.062 e-14 \quad 3.099 e-14$ 9.891e-02 2.477e-14 $1.891 e-14 \quad 2.739 e-14 \quad 3.063 e-14 \quad 3.318 e-14 \quad 3.063 e-14$ 1.307e-01 3.442e-14 $1.479 e-14 \quad 2.164 e-14 \quad 6.068 e-14 \quad 4.945 e-14 \quad 5.557 e-14$ $1.819 e-01 \quad 3.557 e-14 \quad 6.665 e-14 \quad 1.886 e-14 \quad 7.387 e-14 \quad 6.865 e-14 \quad 4.355 e-14$ $2.075 e-01 \quad 3.248 e-14 \quad 1.420 e-14 \quad 5.516 e-14 \quad 1.124 e-13 \quad 4.312 e-14 \quad 5.715 e-14$ 2.417e-01 3.408e-14 $1.024 e-13 \quad 2.750 e-14 \quad 8.113 e-14 \quad 4.199 e-14 \quad 6.509 e-14$ 2.710e-01 $4.302 e-14 \quad 2.030 e-13 \quad 6.031 e-14 \quad 9.246 e-14 \quad 4.383 e-14 \quad 6.137 e-14$ 2.940e-01 $9.336 e-14 \quad 7.858 e-14 \quad 8.159 e-14 \quad 8.863 e-14 \quad 3.392 e-14 \quad 6.710 e-14$ 3.345e-01 7.217e-14 9.314e-14 5.789e-14 1.165e-13 5.470e-14 7.470e-14 3.776e-01 8.331e-14 $1.319 e-13 \quad 6.298 e-14 \quad 1.020 e-13 \quad 6.732 e-14 \quad 8.475 e-14$ 4.234e-01 9.299e-14 6.545e-14 4.890e-14 8.146e-14 7.484e-14 9.520e-14 5.123e-01 $1.082 e-13 \quad 1.336 e-13 \quad 9.842 e-14 \quad 9.902 e-14 \quad 8.681 e-14 \quad 1.110 e-13$ 

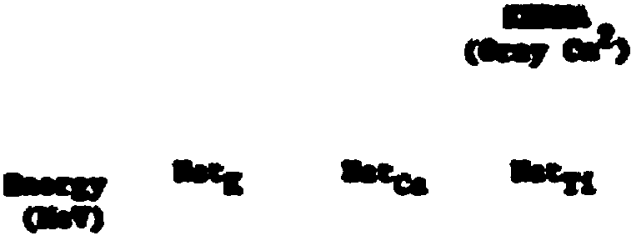

Date

Fon

32

ans

$B$

6.3250-01 $1.2450-131.0010-13$ 1.0140-13 9.506e-14 9.117a-14 1.2580-13 7.3270-01 $1.3970-13$ 2.025e-13 L.CAce-13 $1.395 e-13$ 1.2550-13 $1.3030-13$

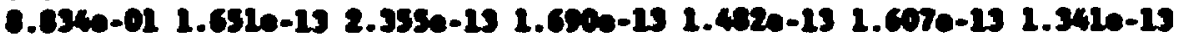
1.0250400 1.005e-13 3.140e-13 $1.9740-13$ 1.956-13 $1.6210-13$ 1.510e-13 $1.1760+002.456-132.9450-13 \quad 1.9510-13 \quad 2.1370-13 \quad 1.6030-13 \quad 1.7600-13$ $1.3320+602.0530-13 \quad 3.7070-132.3950-13 \quad 2.550-132.223 e-13 \quad 2.032 e-13$

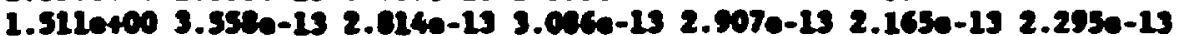
$1.6940+00$ 4.306e-13 $4.019 e-13 \quad 3.1730-13 \quad 3.2640-13 \quad 2.190 e-13 \quad 2.527 e-13$ $1.897 e+60$ 4.975e-13 4.915e-13 $3.113 e-13 \quad 3.532 e-13 \quad 2.616 e-132.664 e-13$ $2.0910+00 \quad 6.2040-135.752 e-13 \quad 3.523 e-13 \quad 3.015 e-13 \quad 3.609 e-132.000 e-13$ $2.305 e+007.039 e-13 \quad 6.441 e-13 \quad 3.909 e-13 \quad 4.156 e-13 \quad 3.591 e-13 \quad 3.043 e-13$ $2.530 e+00$ e.51ee-13 $7.597 e-13 \quad 4.490 e-13 \quad 4.200 e-13 \quad 3.923 e-13 \quad 3.284 e-13$ $2.741 e+00 \quad 9.546 e-139.603 e-134.959 e-134.654 e-13 \quad 4.002 e-13 \quad 3.517 e-13$ $3.011 e+001.109 e-12 \quad 1.291 e-12 \quad 5.146 e-13 \quad 4.765 e-13 \quad 4.137 e-13 \quad 3.767 e-13$ $3.267 e+00 \quad 1.370 e-12 \quad 1.691 e-12 \quad 5.409 e-13 \quad 4.867 e-13 \quad 4.162 e-13 \quad 4.014 e-13$ $3.534 e+001.722 e-12 \quad 2.151 e-12 \quad 5.617 e-13 \quad 5.210 e-13 \quad 4.294 e-13 \quad 4.233 e-13$ 3.811e+00 2.069e-12 2.647e-12 5.804e-13 5.250e-13 4.374e-13 4.443e-13 $4.069 e+002.416 e-12 \quad 2.992 e-125.960 e-13 \quad 5.423 e-134.399 e-134.623 e-13$ $4.396 e+00$ 2.780e-12 $3.340 e-12 \quad 6.201 e-13 \quad 5.571 e-13 \quad 4.607 e-13$ 4.782e-13 $4.704 e+003.075 e-12 \quad 3.681 e-12 \quad 6.588 e-13 \quad 5.706 e-13 \quad 4.883 e-13 \quad 4.918 e-13$ $4.991 e+003.333 e-12 \quad 4.033 e-12 \quad 6.938 e-13 \quad 5.860 e-13 \quad 5.039 e-13 \quad 5.017 e-13$ $5.352 e+00 \quad 3.581 e-12 \quad 4.393 e-12 \quad 7.310 e-13 \quad 5.921 e-13 \quad 5.246 e-13 \quad 5.210 e-13$ $5.658 e+00 \quad 3.813 e-12 \quad 4.729 e-12 \quad 7.682 e-13 \quad 6.015 e-13 \quad 5.509 e-13 \quad 5.494 e-13$ $6.042 e+00 \quad 4.054 e-12 \quad 5.059 e-12 \quad 8.045 e-13 \quad 6.384 a-13 \quad 5.782 e-13 \quad 5.794 e-13$ $6.367 e+00 \quad 4.341 e-12 \quad 5.395 e-12 \quad 8.498 e-13 \quad 6.647 e-13 \quad 6.139 e-13 \quad 6.051 e-13$ $6.737 e+00 \quad 4.647 e-12 \quad 5.737 e-12 \quad 9.019 e-13 \quad 6.959 e-13 \quad 6.566 e-13 \quad 6.261 e-13$ $7.156 e+00 \quad 4.980 e-12 \quad 6.128 e-12 \quad 9.615 e-13 \quad 7.160 e-13 \quad 7.127 e-13 \quad 6.511 e-13$ $7.548 e+00 \quad 5.324 e-12 \quad 6.430 e-12 \quad 1.012 e-12 \quad 7.419 e-13 \quad 7.704 e-13 \quad 6.905 e-13$ $7.910 e+00 \quad 5.639 e-12 \quad 6.678 e-12 \quad 1.059 e-12 \quad 7.644 e-13 \quad 8.300 e-13 \quad 7.313 e-13$ $8.322 e+005.962 e-12 \quad 6.921 e-12 \quad 1.109 e-12 \quad 8.008 e-13 \quad 9.044 e-13 \quad 7.787 e-13$ $8.787 e+00 \quad 6.317 e-12 \quad 7.210 e-12 \quad 1.169 e-12 \quad 8.513 e-13 \quad 1.013 e-12 \quad 8.465 e-13$

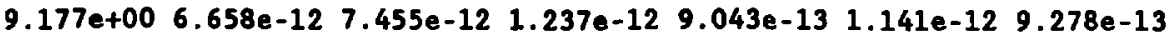

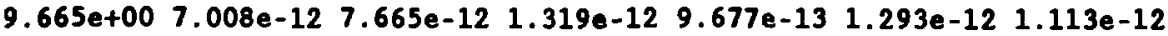

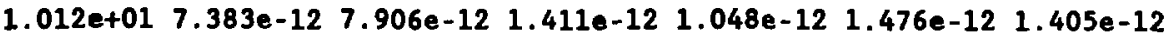
$1.058 e+01 \quad 7.703 e-12 \quad 8.261 e-12 \quad 1.516 e-12 \quad 1.138 e-12 \quad 1.656 e-12 \quad 1.758 e-12$

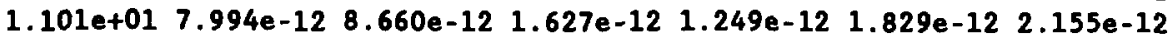

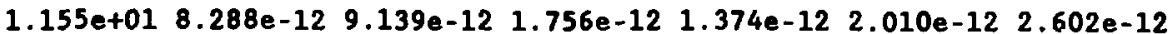
$1.199 e+018.562 e-12 \quad 9.686 e-12 \quad 1.895 e-12 \quad 1.503 e-12 \quad 2.189 e-12 \quad 3.088 e-12$ $1.250 e+01 \quad 8.827 e-12 \quad 1.022 e-11 \quad 2.060 e-12 \quad 1.621 e-12 \quad 2.368 e-12 \quad 3.550 e-12$ $\begin{array}{lllllll}1.307 e+01 & 9.124 e-12 & 1.083 e-11 & 2.272 e-12 & 1.750 e-12 & 2.574 e-12 & 4.046 e-12\end{array}$ $1.354 e+019.363 e-12 \quad 1.153 e-11 \quad 2.455 e-12 \quad 1.890 e-12 \quad 2.725 e-12 \quad 4.561 e-12$ 


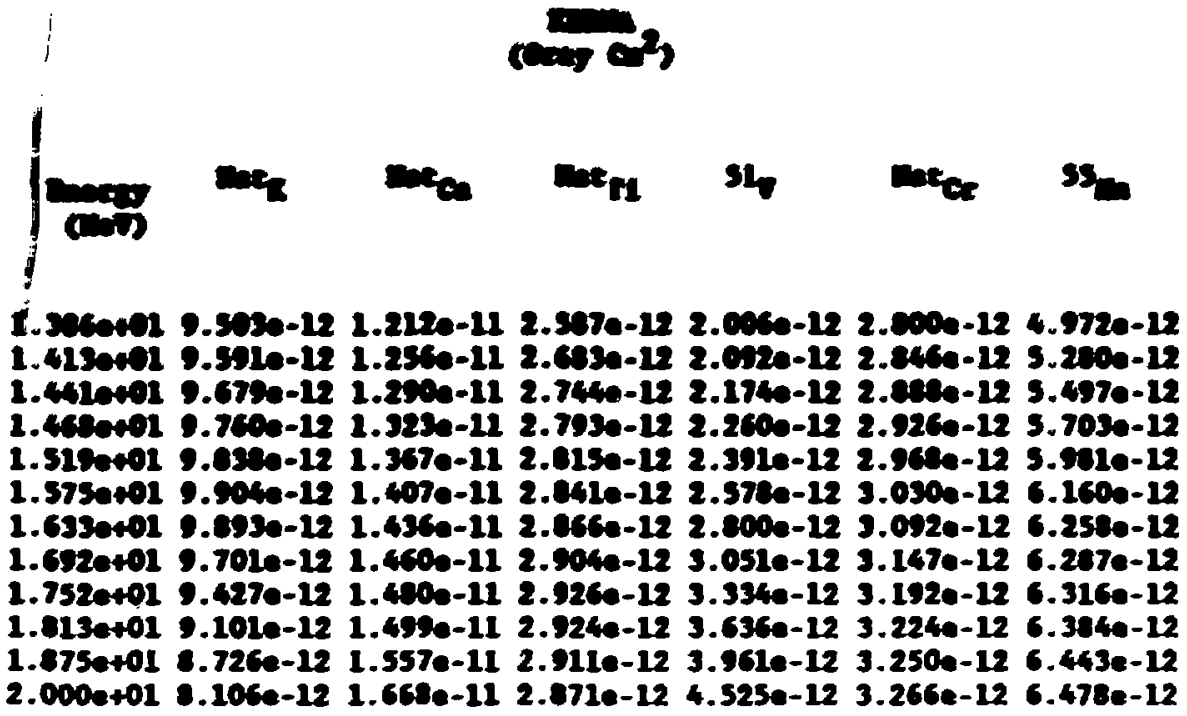




\title{
$\cos 2 x$
}

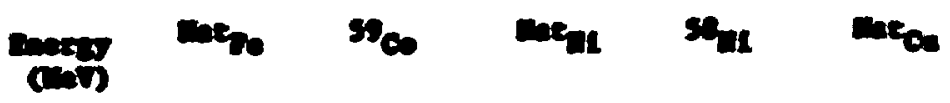

\begin{abstract}
1.307e-68
5.227e-c5 7.452e-15 9.121e-14 $1.475 e-14$ 1.393e-14 e.346e-15

2.cole-65 3.7310-15 4.64ke-14 7.376e-15 7.1940-15 4.200e-15

3.267e-ce 2.453e-15 3.0250-14 4.9200-15 4.843e-15 $2.0040-15$

4.704e-C 2.037e-15 2.4990-14 4.022e-15 4.107e-15 $2.296 e-15$

$0.322 e-65$ 1.600e-15 $1.9900-14$ 3.160e-15 3.293e-15 1.800e-15

1.307e-07 1.242e-15 $1.523 e-14$ 2.463e-15 2.619e-15 1.406e-15

1.82e-07 $1.0140-15$ 1.262e-14 $2.022 e-15$ 2.199e-15 1.14ee-15

2.561e-07 0.622e-16 $1.049 e-14 \quad 1.705 e-15$ 1.891e-15 $9.711 e-16$

3.345a-07 7.463a-16 9.055e-15 $1.475 a-15$ l.673e-15 $\quad 0.405 a-16$

4.234e-07 6.622e-16 $\quad .121 e-15$ 1.295e-15 $1.504 e-15 \quad 7.414 e-16$

5.123e-07 5.904e-16 7.206e-15 1.165e-15 $1.390 e-15$ 6.664a-16

7.527e-07 5.115e-16 6.245e-15 $1.009 e-15$ 1.229e-15 $5.750 e-16$

1.176e-06 4.147e-16 5.111e-15 8.177e-16 1.041e-15 4.672e-16

$1.511 e-06$ 3.521e-16 $4.422 e-15$ 6.947e-16 9.139e-16 3.952e-16

2.091e-06 3.031e-16 3.779e-15 5.978e-16 $8.250 e-16 \quad 3.420 e-16$

2.741e-06 2.639e-16 3.262e-15 5.185e-16 7.438e-16 2.955e-16

3.534e-06 2.309e-16 2.907e-15 4.565e-16 6.809e-16 $2.596 e-16$

4.704e-06 2.026e-16 2.619e-15 3.967e-16 6.259e-16 2.272e-16

5.658e-06 1.807e-16 2.310e-15 3.548e-16 5.852e-16 $2.020 e-16$

6.737e-06 1.660e-16 2.141e-15 3.259e-16 $5.563 e-16 \quad 1.829 e-16$

8.321e-06 1.531e-16 2.001e-15 2.984e-16 5.297e-16 $1.642 e-16$

9.620e-06 $1.401 e-16 \quad 1.850 e-15 \quad 2.739 e-16 \quad 5.053 e-16 \quad 1.490 e-16$

$1.101 e-05 \quad 1.318 e-16 \quad 1.732 e-15 \quad 2.586 e-16 \quad 4.878 e-16 \quad 1.361 e-16$

1.307e-05 $1.248 e-16 \quad 1.672 e-15 \quad 2.443 e-16 \quad 4.754 e-16 \quad 1.182 e-16$

1.468e-05 $1.176 e-16 \quad 1.613 e-15 \quad 2.312 e-16 \quad 4.648 e-16 \quad 1.037 e-16$

1.581e-05 $1.132 e-16 \quad 1.569 e-15 \quad 2.232 e-16 \quad 4.586 e-16 \quad 9.576 e-17$

1.758e-05 $1.126 e-16 \quad 1.523 e-15 \quad 2.156 e-16 \quad 4.529 e-16 \quad 9.119 e-17$

1.882e-05 1.122e-16 $1.475 e-15 \quad 2.077 e-16 \quad 4.470 e-16 \quad 8.720 e-17$

2.075e-05 1.118e-16 $1.427 e-15 \quad 2.015 e-16 \quad 4.430 e-16 \quad 8.344 e-17$

2.277e-05 1.113e-16 $1.404 e-15 \quad 1.957 e-16 \quad 4.399 e-16 \quad 7.830 e-17$

2.417e-05 1.108e-16 $1.394 e-15 \quad 1.915 e-16 \quad 4.381 e-16 \quad 7.432 e-17$

2.561e-05 $1.105 e-16 \quad 1.386 e-15 \quad 1.881 e-16 \quad 4.366 e-16 \quad 7.151 e-17$

$2.710 e-05 \quad 1.100 e-16 \quad 1.378 e-15 \quad 1.847 e-1.6 \quad 4.352 e-16 \quad 6.896 e-17$

$\begin{array}{llllll}2.862 e-05 & 1.097 e-16 & 1.369 e-15 & 1.819 e-16 & 4.346 e-16 & 6.663 e-17\end{array}$

$2.940 e-05 \quad 1.093 e-16 \quad 1.362 e-15 \quad 1.803 e-16 \quad 4.345 e-16 \quad 6.506 e-17$

3.099e-05 $1.087 e-16 \quad 1.359 e-15 \quad 1.787 e-16 \quad 4.346 e-16 \quad 6.369 e-17$

3.345e-05 $1.060 e-16 \quad 1.376 e-15 \quad 1.760 e-16 \quad 4.347 e-16 \quad 6.228 e-17$

3.601e-05 $1.026 e-16 \quad 1.402 e-15 \quad 1.734 e-16 \quad 4.356 e-16 \quad 6.062 e-17$

3.866e-05 9.911e-17 $1.428 e-15 \quad 1.711 e-16 \quad 4.369 e-16 \quad 5.929 e-17$

4.048e-05 9.608e-17 $1.451 e-15 \quad 1.691 e-16 \quad 4.381 e-16 \quad 5.836 e-17$
\end{abstract}




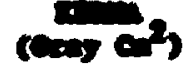

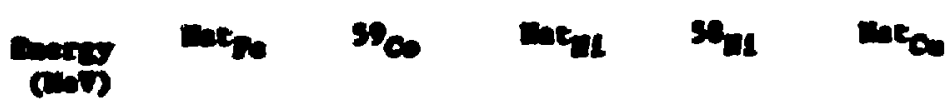

$4.2340-059.357 e-17$ 1.4700-15 $1.677 e-16$ 4.393e-16 $5.7600-17$

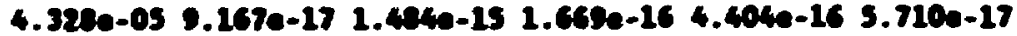
$4.6140-050.9400-171.3040-151.657 e-164.4200-165.655 e-17$ $4.717 e-05$ 0.ence-17 $1.325 e-15$ 1.648e-16 4.43e-16 5.620e-17 $4.9160-050.500 e-171.5410-151.646 e-164.457 e-165.585 e-17$ 5.1230-05 8.9200-17 1.565e-15 $1.6420-164.4760-165.5200-17$ 5.332e-05 8.940e-17 $1.614 e-15 \quad 1.639 e-16$ 4.476e-16 5.345e-17 5.654e-05 8.966e-17 1.603e-15 $1.634 e-16$ 4.474e-16 5.211a-17 $5.763 e-05 \quad 8.987 e-17 \quad 1.739 e-15 \quad 1.630 e-16 \quad 4.472 e-16 \quad 5.100 e-17$ 6.097e-05 9.014e-17 $1.797 e-15$ 1.626e-16 $4.469 e-16 \quad 4.996 a-17$ 6.325e-05 9.131e-17 $1.899 e-15$ 1.624e-16 $4.470 e-16 \quad 4.888 e-17$ 6.557e-05 $9.257 e-17 \quad 1.097 e-15 \quad 1.625 e-16 \quad 4.473 e-16 \quad 4.814 e-17$ 6.674e-05 9.353e-17 $2.073 e-15 \quad 1.626 e-16 \quad 4.475 e-16 \quad 4.769 e-17$ 7.033e-05 $9.484 e-17 \quad 2.176 e-15 \quad 1.627 e-16 \quad 4.477 e-16 \quad 4.718 e-17$ 7.155e-05 $9.615 e-17 \quad 2.294 e-15 \quad 1.627 e-16 \quad 4.480 e-16 \quad 4.693 e-17$ $7.527 e-05$ 9.750e-17 2.473e-15 $1.630 e-16 \quad 4.484 e-16 \quad 4.709 e-17$ $7.780 e-05$ 9.921e-17 2.701e-15 $1.637 e-16 \quad 4.494 e-16 \quad 4.730 e-17$ $7.908 e-05 \quad 1.003 e-16 \quad 2.840 e-15 \quad 1.642 e-16 \quad 4.501 e-16 \quad 4.749 e-17$ 6.167e-05 1.013e-16 $2.997 e-15$ 1.647e-16 4.507e-16 $4.768 e-17$ $8.431 e-05 \quad 1.025 e-16 \quad 3.320 e-15 \quad 1.653 e-16 \quad 4.516 e-16 \quad 4.795 e-17$ 8.834e-05 $1.041 e-16 \quad 3.764 e-15 \quad 1.662 e-16 \quad 4.527 e-16 \quad 4.836 e-17$ 9.108e-05 $1.057 e-16 \quad 4.224 e-15 \quad 1.670 e-16 \quad 4.539 e-16 \quad 4.881 e-17$ 9.386e-05 1.070e-16 4.859e-15 1.679e-16 4.550e-16 4.919e-17 $9.668 e-05 \quad 1.083 e-16 \quad 5.643 e-15 \quad 1.689 e-16 \quad 4.562 e-16 \quad 4.963 e-17$ 9.811e-05 $1.093 e-16 \quad 6.240 e-15 \quad 1.697 e-16 \quad 4.571 e-16 \quad 4.996 e-17$ 1.025e-04 $1.110 e-16 \quad 7.224 e-15 \quad 1.709 e-16 \quad 4.586 e-16 \quad 5.046 e-17$ 1.099e-04 1.167e-16 $1.132 e-14 \quad 1.742 e-16 \quad 4.625 e-16 \quad 5.178 e-17$ 1.176e-04 1.243e-16 2.105e-14 $1.789 e-16 \quad 4.678 e-16 \quad 5.349 e-17$ $\begin{array}{llllll}1.256 e-04 & 1.322 e-16 & 6.544 e-14 & 1.840 e-16 & 4.735 e-16 & 5.525 e-17\end{array}$ $1.338 e-04 \quad 1.403 e-16 \quad 4.813 e-13 \quad 1.892 e-16 \quad 4.795 e-16 \quad 5.705 e-17$ 1.406e-04 1.479e-16 2.282e-13 $1.942 e-16 \quad 4.851 e-16 \quad 5.871 e-17$ 1.511e-04 $1.565 e-16 \quad 3.635 e-14 \quad 2.002 e-16 \quad 4.919 e-16 \quad 6.136 e-17$ 1.601e-04 1.663e-16 $1.155 e-14 \quad 2.070 e-16 \quad 4.996 e-16 \quad 6.477 e-17$ 1.694e-04 1.690e-16 6.059e-15 2.134e-16 5.068e-16 6.920e-17 $1.789 e-041.655 e-16 \quad 4.306 e-15 \quad 2.203 e-16 \quad 5.145 e-16 \quad 8.255 e-17$ 1.887e-04 $1.637 e-16 \quad 3.031 e-15 \quad 2.275 e-16 \quad 5.225 e-16 \quad 1.126 e-16$ 1.988e-04 1.647e-16 1.817e-15 2.348e-16 5.307e-16 $1.679 e-16$ $\begin{array}{llllll}2.091 e-04 & 1.658 e-16 & 1.436 e-15 & 2.427 e-16 & 5.395 e-16 & 2.332 e-16\end{array}$ 2.741e-04 1.885e-16 $7.534 e-16 \quad 2.727 e-16 \quad 5.730 e-16 \quad 3.158 e-16$ 3.267e-04 2.692e-16 3.390e-16 3.208e-16 6.265e-16 $1.889 e-16$ 3.811e-04 3.144e-16 2.421e-16 $3.660 e-16 \quad 6.763 e-16 \quad 1.482 e-16$ 


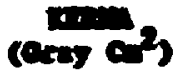

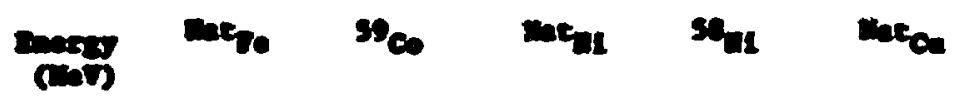

4.704e-04 3.012e-16 1.92\%e-16 4.274e-16 7.443e-16 2.031e-16 4.991e-04 3.030e-16 $1.747 e-16$ 4.783e-16 8.006e-16 3.296e-16 5.652e-04. 3.102e-16 $1.610 e-16$ 5.196e-16 $8.452 e-16 \quad 5.224 a-16$ 6.043e-04. 3.193e-16 $1.523 e-16 \quad 5.657 a-16 \quad 8.970 e-16 \quad 9.812 e-16$ $6.367 e-04 \quad 3.2350-16$ 1.507e-16 $5.968 e-16 \quad 9.313 e-16 \quad 0.090 e-16$ 7.156e-04 3.201e-16 $1.455 e-16$ 6.462e-16 9.057e-16 $6.914 e-16$ e.322e-04 $3.923 e-161.507 e-16 \quad 7.332 e-16 \quad 1.052 e-15 \quad 4.941 e-16$ $9.177 e-0 . \quad 4.92 j e-16 \quad 1.575 e-16 \quad 0.233 e-16 \quad 1.181 e-15 \quad 3.870 e-16$ 1.058e-03 $5.563 e-16 \quad 1.707 e-16 \quad 9.246 e-16 \quad 1.292 e-15 \quad 3.463 e-16$ 1.307e-03 6.381e-16 $1.761 e-16 \quad 1.106 e-15 \quad 1.478 e-15 \quad 4.150 e-16$

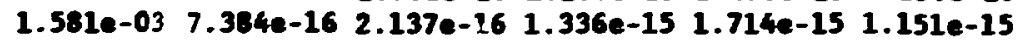
$\begin{array}{llllll}1.882 e-05 & 0.345 e-16 & 1.893 e-16 & 1.595 e-15 & 1.979 e-15 & 2.341 e-15\end{array}$ 2.208e-03 9.273e-16 $2.037 e-16 \quad 1.879 e-15 \quad 2.270 e-15 \quad 3.078 e-15$ 2.561e-03 1.030e-15 2.306e-16 2.459e-15 2.681e-15 1.570e-15 $2.940 e-03 \quad 1.126 e-15 \quad 5.098 e-16 \quad 2.591 e-15 \quad 2.907 e-15 \quad 1.336 e-15$ $3.345 e-03 \quad 1.270 e-15 \quad 3.955 e-16 \quad 3.181 e-15 \quad 3.497 e-15 \quad 9.531 e-16$ 3.777e-03 $1.494 e-15 \quad 9.177 e-16 \quad 4.170 e-15 \quad 4.493 e-15 \quad 9.982 e-16$ 4.234e-03 1.565e-15 $5.602 e-15 \quad 5.982 e-15 \quad 6.339 e-15 \quad 1.478 e-15$

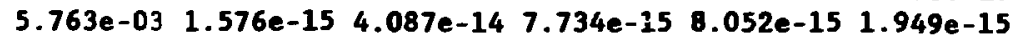

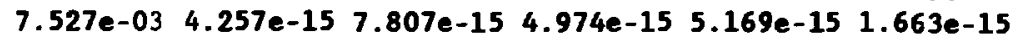
1.025e-02 6.902e-15 $5.187 e-15 \quad 4.481 e-15 \quad 4.817 e-15 \quad 4.167 e-15$ $1.511 e-02 \quad 3.671 e-15 \quad 5.007 e-15 \quad 2.691 e-14 \quad 3.050 e-14 \quad 6.105 e-15$ $2.091 e-02 \quad 2.828 e-15 \quad 6.800 e-15 \quad 5.476 e-14 \quad 5.012 e-14 \quad 8.691 e-15$ $\begin{array}{llllll}2.646 e-02 & 2.872 e-15 & 3.152 e-14 & 2.266 e-14 & 1.952 e-14 & 1.051 e-14\end{array}$ $\begin{array}{llllll}3.267 e-02 & 5.178 e-14 & 2.069 e-14 & 2.835 e-14 & 2.382 e-14 & 1.220 e-14\end{array}$

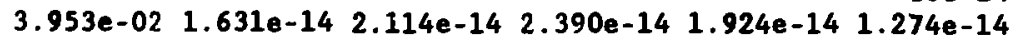
4.704e-02 $1.363 e-14 \quad 1.610 e-14 \quad 2.128 e-14 \quad 1.688 e-14 \quad 1.431 e-14$

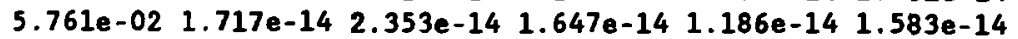

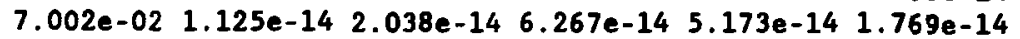
8.322e-02 2.448e-14 $2.563 e-14 \quad 5.192 e-14 \quad 3.479 e-14 \quad 2.064 e-14$ $9.891 e-02 \quad 3.830 e-14 \quad 4.100 e-14 \quad 5.157 e-14 \quad 2.811 e-14 \quad 2.379 e-14$ $\begin{array}{llllll}1.307 e-01 & 2.041 e-14 & 4.403 e-14 & 3.538 e-14 & 3.243 e-14 & 2.875 e-14\end{array}$ $1.819 e-013.631 e-14 \quad 3.476 e-14 \quad 4.591 e-14 \quad 5.709 e-14 \quad 3.690 e-14$ $2.075 e-01 \quad 5.857 e-14 \quad 5.092 e-14 \quad 5.277 e-14 \quad 6.329 e-14 \quad 4.356 e-14$ $2.417 \mathrm{e}-01 \quad 3.530 \mathrm{e}-14 \quad 4.609 \mathrm{e}-14 \quad 7.622 \mathrm{e}-14 \quad 8.728 \mathrm{e}-14 \quad 4.859 \mathrm{e}-14$ $2.710 e-013.396 e-14 \quad 5.295 e-14 \quad 6.096 e-14 \quad 7.064 e-14 \quad 5.345 e-14$ $2.940 e-01 \quad 4.639 e-14 \quad 6.079 e-14 \quad 9.072 e-14 \quad 1.064 e-13 \quad 5.720 e-14$ 3.345e-01 4.440e-14 6.756e-14 7.419e-14 8.526e-14 6.127e-14 $3.776 e-01 \quad 4.467 e-14 \quad 7.785 e-14 \quad 7.638 e-14 \quad 8.813 e-14 \quad 6.707 e-14$ 4.234e-01 $1.243 e-13 \quad 6.977 e-14 \quad 5.807 e-14 \quad 6.607 e-14 \quad 7.418 \mathrm{e}-14$ 5.123e-01 $1.008 e-13 \quad 9.926 e-14 \quad 9.707 e-14 \quad 1.010 e-13 \quad 8.413 e-14$ 


\section{$(\cos 2)$}

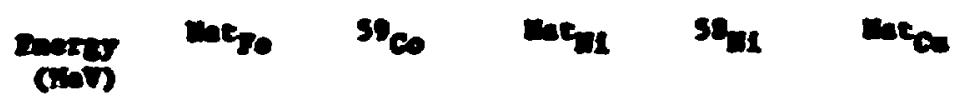

6.3250-C1 $7.5310-14 \quad 1.0120-13 \quad 9.265 e-14 \quad 0.3020-14 \quad 0.7750-14$ $7.527 e-01 \quad 9.8540-14 \quad 1.2310-13 \quad 1.046 e-13 \quad 1.0620-131.1360-13$ 0.0340-01 $1.317 e-13 \quad 1.2490-23 \quad 1.244 e-13 \quad 1.3140-13 \quad 1.2000-13$ $1.0250+001.013 e-13 \quad 1.495 e-13 \quad 1.4040-13 \quad 1.567 e-13 \quad 1.406 e-13$ $1.17604001 .206 e-13 \quad 1.6140-13 \quad 1.633 e-13 \quad 1.742 e-131.553 e-13$ $1.3380+001.7350-13 \quad 1.022 e-13 \quad 2.1930-13 \quad 2.111 e-13 \quad 1.669 e-13$ $1.51104001 .6420-13 \quad 1.0420-13 \quad 2.2950-13 \quad 2.504 e-13 \quad 1.670 e-13$ $\begin{array}{lllllll}1.694 e+00 & 2.176 e-13 & 2.035 e-13 & 2.392 e-13 & 2.825 e-13 & 1.764 e-13\end{array}$ $\begin{array}{lllllll}1.097 e+00 & 2.197 e-13 & 2.099 e-13 & 2.576 e-13 & 3.179 e-13 & 1.909 e-13\end{array}$ $2.091 e+002.679 e-13 \quad 2.217 e-13 \quad 2.971 e-13 \quad 3.605 e-13 \quad 2.071 e-13$ $2.305 e+002.823 e-13 \quad 2.399 e-13 \quad 3.429 e-13 \quad 4.189 e-13 \quad 2.303 e-13$ $2.530 e+00 \quad 3.416 e-13 \quad 2.616 e-13 \quad 4.229 e-13 \quad 4.503 e-13 \quad 2.555 e-13$ $2.741 e+00 \quad 3.852 e-13 \quad 2.761 e-13 \quad 4.774 e-13 \quad 5.496 e-13 \quad 2.830 e-13$ $3.011 e+003.828 e-13 \quad 2.963 e-13 \quad 5.775 e-13 \quad 7.544 e-13 \quad 3.135 e-13$ $3.267 e+00 \quad 4.267 e-13 \quad 3.335 e-13 \quad 6.520 e-13 \quad 9.886 e-13 \quad 3.469 e-13$ $\begin{array}{llllll}3.534 e+00 & 4.479 e-13 & 3.538 e-13 & 7.846 e-13 & 9.345 e-13 & 3.795 e-13\end{array}$ $3.811 e+00 \quad 4.802 e-13 \quad 3.743 e-13 \quad 9.437 e-13 \quad 9.573 e-13 \quad 4.119 e-13$ $4.069 e+00 \quad 5.290 e-13 \quad 3.820 e-13 \quad 1.071 e-12 \quad 1.227 e-12 \quad 4.422 e-13$ $4.396 e+00 \quad 5.761 e-13 \quad 4.039 e-13 \quad 1.233 e-12 \quad 1.449 e-12 \quad 4.737 e-13$ $4.704 e+00 \quad 5.997 e-13 \quad 4.349 e-13 \quad 1.376 e-12 \quad 1.713 e-12 \quad 5.076 e-13$ $4.991 e+00 \quad 6.118 e-13 \quad 4.629 e-13 \quad 1.515 e-12 \quad 1.980 e-12 \quad 5.385 e-13$ $\begin{array}{llllll}5.352 e+00 & 6.561 e-13 & 4.900 e-13 & 1.749 e-12 & 2.249 e-12 & 5.726 e-13\end{array}$ $5.658 e+007.260 e-13 \quad 5.167 e-13 \quad 2.004 e-12 \quad 2.501 e-12 \quad 6.110 e-13$ $6.042 e+00 \quad 8.154 e-13 \quad 5.402 e-13 \quad 2.253 e-12 \quad 2.775 e-12 \quad 6.552 e-13$ $\begin{array}{llllll}6.367 e+00 & 9.096 e-13 & 5.749 e-13 & 2.504 e-12 & 3.028 e-12 & 7.064 e-13\end{array}$ $6.737 e+00 \quad 1.007 e-12 \quad 6.193 e-13 \quad 2.737 e-12 \quad 3.252 e-12 \quad 7.596 e-13$ $7.156 e+00 \quad 1.110 e-12 \quad 6.739 e-13 \quad 2.980 e-12 \quad 3.513 e-12 \quad 8.207 e-13$ $7.548 e+001.258 e-12 \quad 7.402 e-13 \quad 3.207 e-12 \quad 3.769 e-12 \quad 8.720 e-13$ $7.910 e+0.01 .403 e-12 \quad 8.106 e-13 \quad 3.400 e-12 \quad 4.014 e-12 \quad 9.166 e-13$ 8. $322 e+001.546 e-12 \quad 8.900 e-13 \quad 3.600 e-12 \quad 4.275 e-12 \quad 9.804 e-13$ 8.787e+00 $1.703 e-12 \quad 9.913 e-13 \quad 3.849 e-12 \quad 4.580 e-12 \quad 1.092 e-12$ $9.177 e+001.863 e-12 \quad 1.093 e-12 \quad 4.111 e-12 \quad 4.899 e-12 \quad 1.216 e-12$ $9.665 e+00 \quad 2.022 e-12 \quad 1.184 e-12 \quad 4.371 e-12 \quad 5.232 e-12 \quad 1.359 e-12$ $1.012 e+01 \quad 2.183 e-12 \quad 1.281 e-12 \quad 4.644 e-12 \quad 5.626 e-12 \quad 1.534 e-12$ $\begin{array}{lllllll}1.058 e+01 & 2.334 e-12 & 1.369 e-12 & 4.932 e-12 & 6.011 e-12 & 1.696 e-12\end{array}$ $\begin{array}{llllll}1.101 e+01 & 2.484 e-12 & 1.454 e-12 & 5.208 e-12 & 6.411 e-12 & 1.859 e-12\end{array}$ $\begin{array}{llllll}1.155 e+01 & 2.646 e-12 & 1.543 e-12 & 5.508 e-12 & 6.792 e-12 & 2.035 e-12\end{array}$ $1.199 e+01 \quad 2.815 e-12 \quad 1.641 e-12 \quad 5.790 e-12 \quad 7.128 e-12 \quad 2.216 e-12$

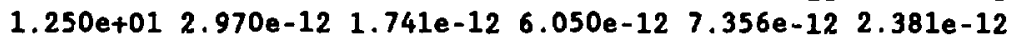
$1.307 e+01 \quad 3.136 e-12 \quad 1.838 \mathrm{e}-12 \quad 6.292 \mathrm{e}-12 \quad 7.627 \mathrm{e}-12 \quad 2.535 \mathrm{e}-12$ $1.354 e+01 \quad 3.287 e-12 \quad 1.923 e-12 \quad 6.509 e-12 \quad 8.012 e-12 \quad 2.678 e-12$ 


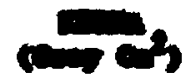

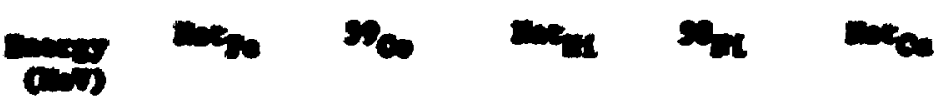

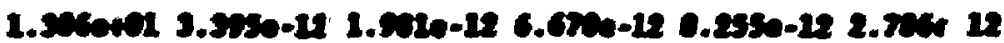

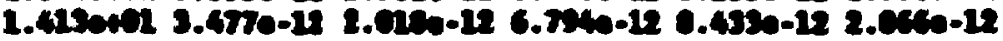

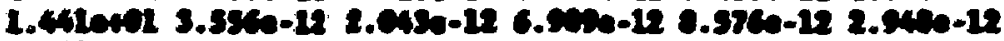

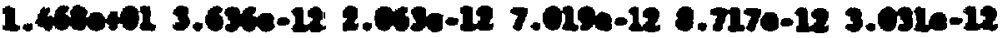

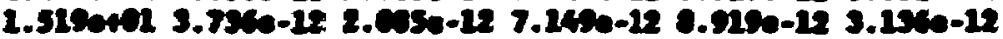

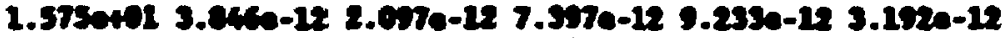

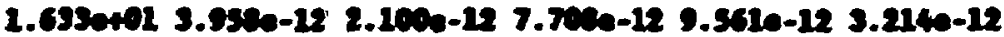

1.6920+e1 4.073e-12 2.093e-12 0.16e-12 9.029e-12 3.2120-12

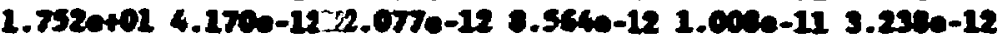

1.013e+01 4.274e-12:2.05se-12 9.007e-12 1.032e-11 3.318e-12

1.875e+01 4.371e-12 $2.0410-12$ 9.50se-12 1.054e-11 $3.3450-12$

2.000e+01 4.501e-12 $2.015 e-12$ l.036e-11 $1.046 e-11$ 3.336e-12 


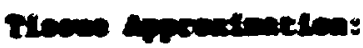
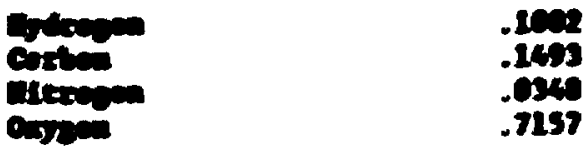

nane (Rems):
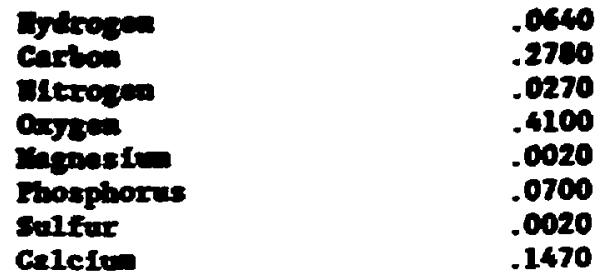

Hascle (ICRJ):

$\begin{array}{ll}\text { Eydrogen } & .1020 \\ \text { Carbon } & .1230 \\ \text { Mitrogen } & .0350 \\ \text { Orgren } & .7290 \\ \text { Sodiun } & .0008 \\ \text { Ingmesiun } & .0002 \\ \text { Phosphorus } & .0020 \\ \text { Sulfur } & .0050 \\ \text { Potassius } & .0030 \\ \text { Calciun } & .0001\end{array}$

Standard Man:

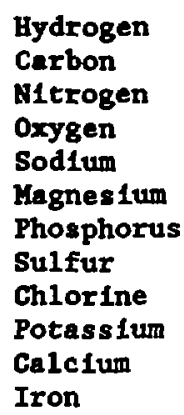

.1000

.1800

.0300

.6500

.0015

.0005

.0100

.0025

.0015

.0020

.0150

.0070 


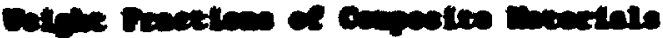

4-LS rinete:

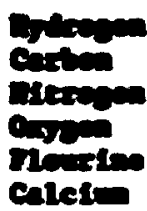

.0382

.032

.0274

.019

How 2ype or 6/0:

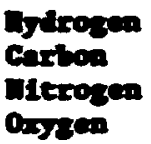

.0500

Incite:

$\begin{array}{ll}\text { Bydrogen } & .0805 \\ \text { Crrbon } & .5998 \\ \text { Oxygen } & .3196\end{array}$

Liquid, Huscle Equivalent:

$$
\begin{aligned}
& \text { Hydrogen } \\
& \text { Crrbon } \\
& \text { Mitrogen } \\
& \text { Oxygen }
\end{aligned}
$$

.1020

Hater:

Hydrogen

Oxygen

Acetylene:

Hydrogen

Carbon

A1r, Dry:

$$
\begin{aligned}
& \text { Carbon } \\
& \text { istrogen } \\
& \text { Oxygen } \\
& \text { Argon }
\end{aligned}
$$$$
.0001
$$$$
.7552
$$$$
.2318
$$$$
.0129
$$ 


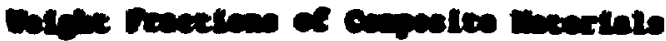

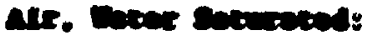

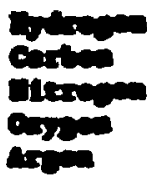

.015

-cons

.745

.2413

.027

Cartor Dleztle:

$$
\text { Coster }
$$$$
.2729
$$

.7271

Ethyleas:

Ijolrogen

.2437

Carbon

.8563

II Ges. with Kethrne:

Hydrogen

.1019

Carbon

.4562

Eitrogen

.0352

Oxysen

.4068 